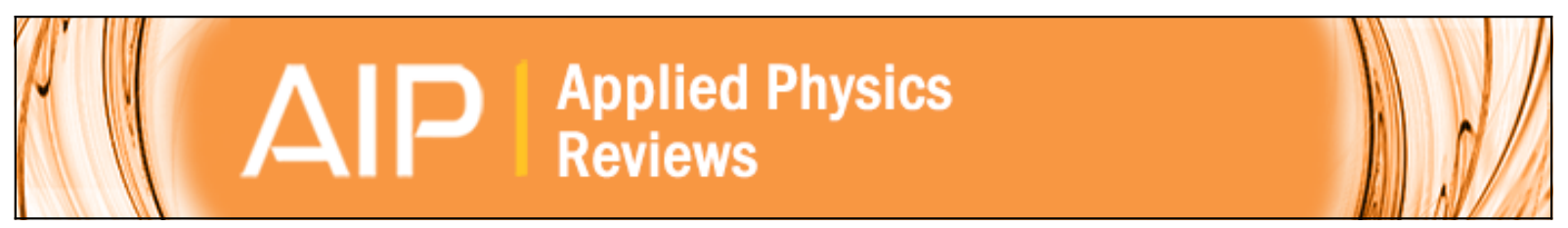

\title{
Optical nonlinearities of small polarons in lithium niobate
}

Mirco Imlau, Holger Badorreck, and Christoph Merschjann

Citation: Applied Physics Reviews 2, 040606 (2015); doi: 10.1063/1.4931396

View online: http://dx.doi.org/10.1063/1.4931396

View Table of Contents: http://scitation.aip.org/content/aip/journal/apr2/2/4?ver=pdfcov

Published by the AIP Publishing

\section{Articles you may be interested in}

Thermal stability of a slab waveguide implemented by a particles implantation in potassium lithium tantalate niobate

Appl. Phys. Lett. 87, 251917 (2005); 10.1063/1.2147723

Anisotropic properties of ultrafast laser-driven microexplosions in lithium niobate crystal

Appl. Phys. Lett. 87, 241107 (2005); 10.1063/1.2142083

Temperature dependence of the thermo-optic coefficient of lithium niobate, from 300 to $515 \mathrm{~K}$ in the visible and infrared regions

J. Appl. Phys. 98, 036101 (2005); 10.1063/1.1988987

Predicting temperature dependence of the refractive index and nonlinear optical coefficients in lithium niobate

J. Appl. Phys. 92, 4638 (2002); 10.1063/1.1510173

Lifetime of small polarons in iron-doped lithium-niobate crystals

J. Appl. Phys. 87, 1034 (2000); 10.1063/1.371976

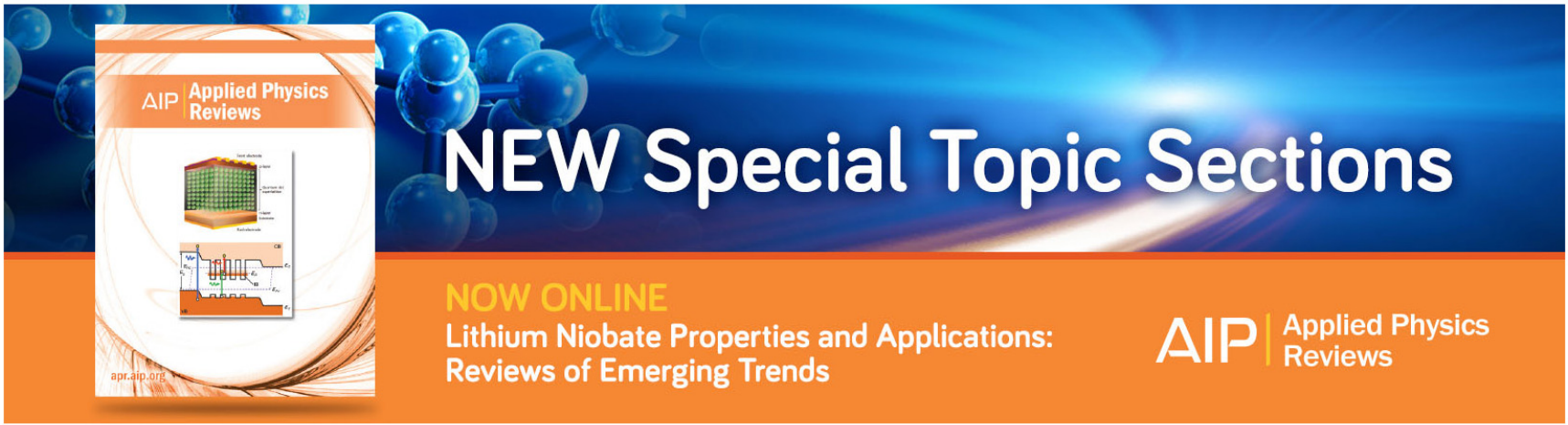




\title{
APPLIED PHYSICS REVIEWS
}

\section{Optical nonlinearities of small polarons in lithium niobate}

\author{
Mirco Imlau, ${ }^{1, a)}$ Holger Badorreck, ${ }^{1}$ and Christoph Merschjann ${ }^{2}$ \\ ${ }^{1}$ Physics Department, Osnabrueck University, Barbarastr. 7, 49076 Osnabrueck, Germany \\ ${ }^{2}$ Fachbereich Physik, Freie Universität Berlin, Arnimallee 14, 14195 Berlin, Germany
}

(Received 21 April 2015; accepted 28 May 2015; published online 20 October 2015)

An overview of optical nonlinearities of small bound polarons is given, which can occur in the congruently melting composition of $\mathrm{LiNbO}_{3}$. Such polarons decisively influence the linear and nonlinear optical performance of this material that is important for the field of optics and photonics. On the basis of an elementary phenomenological approach, the localization of carriers in a periodic lattice with intrinsic defects is introduced. It is applied to describe the binding energies of four electron and hole small polarons in $\mathrm{LiNbO}_{3}$ : small free $\mathrm{Nb}_{\mathrm{Nb}}^{4+}$ polarons, small bound $\mathrm{Nb}_{\mathrm{Li}}^{4+}$ polarons, small bound $\mathrm{Nb}_{\mathrm{Li}}^{4+}: \mathrm{Nb}_{\mathrm{Nb}}^{4+}$ bipolarons, and small bound $\mathrm{O}^{-}$hole polarons. For the understanding of their linear interaction with light, an optically induced transfer between nearest-neighboring polaronic sites is assumed. It reveals spectrally well separated optical absorption features in the visible and near-infrared spectral range, their small polaron peak energies and lineshapes. Nonlinear interaction of light is assigned to the optical formation of short-lived small polarons as a result of carrier excitation by means of band-to-band transitions. It is accompanied by the appearance of a transient absorption being spectrally constituted by the individual fingerprints of the small polarons involved. The relaxation dynamics of the transients is thermally activated and characterized phenomenologically by a stretched exponential behavior, according to incoherent 3D small polaron hopping between regular and defect sites of the crystal lattice. It is shown that the analysis of the dynamics is a useful tool for revealing the recombination processes between small polarons of different charge. Nonlinear interaction of small polarons with light furthermore results in changes of the index of refraction. Besides its causal relation to the transients via KramersKronig relation, pronounced index changes may occur due to optically generated electric fields modulating the index of refraction via the linear electro-optic effect, also. Based on a microscopic picture and by considering the local structural environment of bound polarons, the appearance of photovoltaic currents is explained straightforwardly as a result of the optically induced carrier transfer. Both transient absorption and index changes are spatially confined to the intensity profile of the interacting light allowing for the recording of efficient mixed absorption and phase volume holograms. By means of holographic spectroscopy, these small-polaron based optical nonlinearities are verified either without or with the action of the linear electro-optic effect; their prominent features are highlighted by appropriate experimental studies wherin the ultrafast response on the picosecond time scale is the most recognized one. Based on these findings, the consequences for applications of $\mathrm{LiNbO}_{3}$ in the field of nonlinear optics and photonics are presented. Besides visionary examples like real-time, 3D holographic displays, the impact of optical nonlinearities of small polarons for present applications are discussed with frequency conversion and respective limiting effects, such as green-induced infrared absorption and optical damage, as important example. (C) 2015 AIP Publishing LLC. [http://dx.doi.org/10.1063/1.4931396]

\section{TABLE OF CONTENTS}

I. INTRODUCTION

II. STRUCTURE PHYSICS OF SMALL

POLARONS IN LITHIUM NIOBATE. ........

A. Holsteins' molecular-crystal model ........

B. Small free and bound polarons in $\mathrm{LiNbO}_{3}$..

a)mirco.imlau@uni-osnabrueck.de

III. OPTICAL ABSORPTION FEATURES OF SMALL POLARONS ....................

A. Modelling ................... 5

B. Experimental fingerprints............ 6

IV. OPTICAL GENERATION OF SMALL POLARONS..................... 6

A. Light-induced small polaron absorption .... 8

1. Fundamental description........... 8

2. Thermally activated decay .......... 8

B. Experimental examples.............. 9 
1. Number densities of optically generated

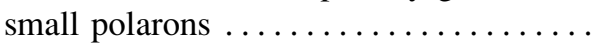

2. Recombination dynamics of optically generated small polarons ...........

3. Optical formation time of small polarons

V. OPTICAL EXCITATION FROM POLARONIC

CENTERS . . . . . . . . . . . . . . .

A. Directed optical transfer of small polarons

from $\mathrm{Fe}_{\mathrm{Li}} \ldots \ldots \ldots \ldots \ldots \ldots \ldots \ldots \ldots$

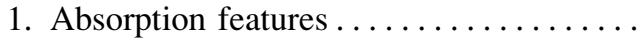

2. Net currents $\ldots \ldots \ldots \ldots \ldots \ldots \ldots \ldots$

VI. HOLOGRAM RECORDING BY MEANS OF

SMALL POLARONS...............

A. Population density holograms .........

1. Hologram recording mechanism ........

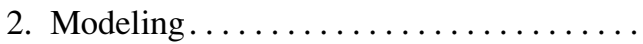

3. Results and analysis $\ldots \ldots \ldots \ldots \ldots \ldots$

B. Holograms by means of the electro-optic

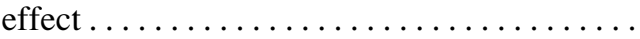

1. Results...................

VII. IMPACT ON NONLINEAR PHOTONICS

AND CONCLUSION ................

A. Developing fields $\ldots \ldots \ldots \ldots \ldots \ldots \ldots$

B. Present applications..............

\section{INTRODUCTION}

Various manifestations of small polarons ${ }^{1,2}$ affect strongly the linear and nonlinear optical properties in the oxide crystal lithium niobate, $\mathrm{LiNbO}_{3}:{ }^{3}$ free electron $\mathrm{Nb}_{\mathrm{Nb}}^{4+}$ polarons, polarons bound to antisite defects, $\mathrm{Nb}_{\mathrm{Li}}^{4+}$, and $\mathrm{Nb}_{\mathrm{Nb}}^{4+}: \mathrm{Nb}_{\mathrm{Li}}^{4+}$ bipolarons. ${ }^{4}$ In addition to these three types of electron polarons, also $\mathrm{O}^{-}$hole small polarons bound to $\mathrm{Li}^{-}$ vacancies can occur. ${ }^{5}$ Such polarons show strong absorption bands peaked near $1.0 \mathrm{eV}\left(\mathrm{Nb}_{\mathrm{Nb}}^{4+}\right), 1.6 \mathrm{eV}\left(\mathrm{Nb}_{\mathrm{Li}}^{4+}\right)$, and $2.5 \mathrm{eV}$ (both, $\mathrm{Nb}_{\mathrm{Nb}}^{4+}: \mathrm{Nb}_{\mathrm{Li}}^{4+}$ and $\mathrm{O}^{-}$). These peak energies are related to the respective polaron stabilization energies $E_{\mathrm{p}}$ and, in addition, to the binding defect potentials for the bound species. $^{6,7}$ The absorption bands are rather asymmetric with pronounced high energy tails, having half-widths of typically one electron volt. Thus, they fall within the large band gap of lithium niobate $\left(\alpha=20 \mathrm{~cm}^{-1}\right.$ at $\approx 3.8 \mathrm{eV}$ (Ref. 8$)$ ) over a wide spectral range.

Using laser light, short-lived small polarons are generated in nominally undoped $\mathrm{LiNbO}_{3}$ by optical interband excitation of carriers and/or by carrier-release via photoionization of extrinsic impurity centers. ${ }^{9}$ Particularly, free polarons, $\mathrm{Nb}_{\mathrm{Nb}}^{4+}$, bound polarons, $\mathrm{Nb}_{\mathrm{Li}}^{4+}$, and $\mathrm{O}^{-}$hole polarons are formed and yield a pronounced transient small polaron absorption in the visible and near-infrared spectral range. Besides, laser-induced dissociation of bipolarons, $\mathrm{Nb}_{\mathrm{Nb}}^{4+}: \mathrm{Nb}_{\mathrm{Li}}^{4+}$, that can be created as stable species at room temperature by raising the Fermi level, e.g., by chemical reduction, leads to the formation of pairs of bound polarons, $\mathrm{Nb}_{\mathrm{Li}}^{4+}$, and free polarons, $\mathrm{Nb}_{\mathrm{Nb}}^{4+}$. All optically generated small polarons recombine within several micro- and milli-seconds at room temperature, either with themselves for charge compensation or with intrinsic $\mathrm{Nb}_{\mathrm{Li}}$ defect centers. The generation of small polarons without the action of light but by heating is possible, e.g., considerable $\mathrm{Nb}_{\mathrm{Nb}}^{4+}$ are present in thermal equilibrium at rather elevated temperatures. ${ }^{10}$

Number densities of optically generated small polarons reach up to tens of $10^{22} \mathrm{~m}^{-3}$ and strongly absorb light in the near-infrared and visible spectral range taking into account the typical polaron absorption cross sections exceeding $4 \cdot 10^{-22} \mathrm{~m}^{2} \cdot{ }^{11-13}$ In recent years, this particular type of transient absorption, assigned to small polaron generation, has been applied for purposes of spectroscopic studies of carrier localization phenomena in lithium niobate related to both electrons and holes. ${ }^{14,15}$ These studies already uncovered the impact of the intrinsic defect structure on the small polaron formation. A time delay between free-carrier excitation and small polaron localization of about hundred femtoseconds at room temperature was found ${ }^{16,17}$ and a detailed model approach to the thermally-activated diffusive hopping of small polarons upon their formation could be deduced. ${ }^{18,19}$

Furthermore, the optical formation of small polarons is accompanied by pronounced changes of the index of refraction, either causally by considering Kramers-Kronig relation $^{20,21}$ or via the small-polaron based photovoltaic effect ${ }^{22}$ and the action of the linear electro-optical effect (Pockels effect). Only recently it was shown that the optical interaction of light with charges localized as small polarons at intrinsic defects and a distorted structural environment may result in the formation of a non-zero net current and the build-up of huge internal electric fields. ${ }^{22}$ This bulk photovoltaic effect $\mathrm{t}^{23-27}$ is one of the most important features of doped $\mathrm{LiNbO}_{3}$, i.e., crystals with extrinsic defect centers, such as $\mathrm{Fe}_{\mathrm{Li}}$. It is because it represents an important step within the cascade of charge-transport phenomena ${ }^{28,29}$ that result in the photorefractive effect, ${ }^{30-33}$ allowing for a variety of photorefractive phenomena in various photovoltaic ferroelectrics. ${ }^{34}$ Due to the application of continuous-wave laser light, a phenomenological description of the photovoltaic effect served convincingly over the last decades. With the introduction of high-power, (ultra-)short pulse laser systems, and the appearance of novel nonlinear phenomena in nominally undoped $\mathrm{LiNbO}_{3}$ (e.g., the appearance of transient absorption with stretched exponential decay ${ }^{15}$ ), a microscopic analysis of light-matter-interaction became inevitably necessary. ${ }^{22}$ Its relation to optically induced index changes in nominally undoped $\mathrm{LiNbO}_{3}$ is studied comprehensively by means of holographic spectroscopy ${ }^{35,36}$ and using short, intense laser pulses. Hologram recording with a single nanosecond laser pulse and efficiencies $>20 \%$ define a remarkable small-polaron based photosensitive response of $S=8 \mathrm{~J} /$ $\mathrm{cm}^{2}$ (Ref. 36) over the visible and near-infrared spectral range. According to the metastable nature of optically generated small polarons, respective holograms decay within micro- and milli-seconds at room temperature and show stretched-exponential decay dynamics. They thus superimpose with the optical nonlinearities induced by two-photon absorption and Drude-Lorentz nonlinearity in $\mathrm{LiNbO}_{3}$ observed instantaneously with the laser pulse. ${ }^{37}$

Obviously, the optical generation of small polarons and their nonlinear optical features must be considered for the various fields of applications of lithium niobate in nonlinear photonics, mainly based on its acousto-optical, nonlinear 
optical, electro-optical, and photorefractive properties. ${ }^{33,38-40}$ Already, it has been shown that small bound polarons may act as intermediate shallow traps in a multistep recording scheme for phase holograms featuring nondestructive read-out. ${ }^{41,42}$ Because of its increasing role as frequency converter, laser-induced damage mechanisms in lithium niobate needs to be analyzed in the framework of small polarons and, particularly, of their optical nonlinearities. The striking role of charged point defects is reported in several prominent oxides widely applied for frequency conversion. ${ }^{43-45}$ Among the materials showing polaronic effects are $\mathrm{KNbO}_{3},{ }^{46} \mathrm{LiTaO}_{3},{ }^{47} \mathrm{KTiOPO}_{4},{ }^{48} \mathrm{LiB}_{3} \mathrm{O}_{5},{ }^{49}$ and $\beta$ - $\mathrm{BaB}_{2} \mathrm{O}_{4}{ }^{45}$ Especially, the latter three materials are technologically of utmost importance for high-power laser applications. $\mathrm{LiNbO}_{3}$ itself, particularly periodically poled $\mathrm{LiNbO}_{3}$ (PPLN), plays an important role for low-power, tunable frequency conversion.

In order to deal adequately with the features of small polarons in the numerous possible optical applications of $\mathrm{LiNbO}_{3}$, it is essential to understand their microscopic structures and the related nonlinear optical properties, which is the aim of this review. $\mathrm{LiNbO}_{3}$ here serves as reference material due to its variety of small polaron features, the possibility of targeted optical formation of small polarons, and pronounced nonlinearities of the crystal itself.

This article is organized as follows: In Section II, the four types of electron and hole polarons are presented from the structural viewpoint allowing for the description of binding by Holsteins' Molecular-Crystal Model (MCM) ${ }^{50}$ The groundstate optical features, such as the respective small polaron peak energies and the spectral dependencies, are discussed in Section III based on an optically induced charge transfer between next-neighboring polaronic sites. The generation of small polarons by optical means is in the focus of Section IV. Here, band-to-band excitation of electron-hole pairs, the excitation from extrinsic defect centers, and the optical dissociation of bipolarons are presented. The relation of number densities to the appearance of transient absorption is derived. Models for the appearance of a stretched exponential decay within the recombination process are summarized. This Section is supported by experimental results on the typical transients over the visible and near-infrared spectral range and of ultra-fast spectroscopic studies that reveal the small polaron formation time. Section V summarizes the microscopic approach of the small-polaron based effect. Besides the appearance of a net current due to a local asymmetry of the $\mathrm{Nb}_{\mathrm{Li}}$ point defect under investigation, the possibility for the excitation of carriers with energies resonant with bandlike states is discussed. Thereby, ballistic transport to distances over the first coordination sphere becomes possible. The appearance of large photovoltaic fields is explained by the superposition of coherent and incoherent transport mechanisms with different mobilities. These fields are shown to modulate the index of refraction via the linear electrooptic effect in Section VI. Besides, population density gratings of small polarons are modeled and verified by experimental results using ns-pulses for the recording of elementary gratings and holographic spectroscopy. These findings are analyzed in the framework of applications of
$\mathrm{LiNbO}_{3}$ in Section VII. With frequency conversion as an example, the role of small polarons in optical damage scenario as well as the possibilities to reduce appropriate effects by a detailed knowledge of optical nonlinearities of small polarons are demonstrated, thereby showing their impact.

\section{STRUCTURE PHYSICS OF SMALL POLARONS IN LITHIUM NIOBATE}

Self-trapped charge carriers were introduced by Landau in 1933 to explain optical properties of alkali halides. ${ }^{51}$ According to Holstein, self-trapping may occur if the charge carrier (electron or hole) distorts the surrounding lattice via Coulomb and short-range interactions. ${ }^{50,52}$ The displacements of the surrounding ions lead to a potential well in which the otherwise free carrier is localized. The quasiparticle composed of the self-trapped carrier and the accompanying pattern of ionic displacements is called polaron. ${ }^{53}$ With increasing coupling strength, the polaronic radius shrinks, eventually restricting the potential well to a single lattice site, a so-called small polaron. Following the definition of Emin, ${ }^{1}$ small polarons thus are characterized by strong coupling as it is the case for large polarons and are distinguished from weakly-coupled polarons.

\section{A. Holsteins' molecular-crystal model}

It is possible to describe the main features of this quasiparticle using Holstein's one-dimensional MCM. ${ }^{50}$ This model describes the polaron localized at one cation site in a threedimensional crystal by an appropriate relaxation of its neighbor ions. Therefore, the most simple conceivable arrangement is the following: Instead of a number of neighbors in a real crystal, only two of them are assumed, forming a one-dimensional chain. The polaron, localized at site $p$, is then assumed to be stabilized by a change of the distance of the partners in the molecule, $q_{p}$. This concept is depicted in Fig. 1.

The results of the one-dimensional model can be transferred to realistic three-dimensional crystals ${ }^{54}$ using slight corrections which will be marked upon occurrence. The following considerations (see Fig. 2) are essentially based on the works of Holstein, ${ }^{50,52}$ Appel, ${ }^{55}$ and Austin and Mott. ${ }^{54}$ For more detailed studies, the reader is referred to those articles.

One may begin with a situation where an excess electron is placed at an originally undistorted site $(q=0)$. The electronic energy at $q$ is then changed by $E_{\mathrm{K}}=-F q$, where $F$ denotes the part of the electron-lattice coupling force that consists of short-range interaction. ${ }^{56}$ At the same time, the elastic energy is enhanced due to the induced lattice distortion: $V_{\mathrm{el}}=1 / 2 K q^{2}$. The total energy of the system is given as the sum of electronic and elastic energies $E_{\mathrm{tot}}=1 / 2 K q^{2}$ $-F q$. By minimizing this energy $\left(q_{\min }=F / K\right)$, one obtains the polaron energy $E_{\mathrm{P}}$

$$
E_{\min }=-\frac{1}{2} \frac{F^{2}}{K}=-E_{\mathrm{P}}
$$

The case of strong electron-phonon coupling is present, i.e., a small polaron, if the energy $E_{\mathrm{P}}$ becomes large. As derived, the electron can be localized at any site of the crystal lattice. 


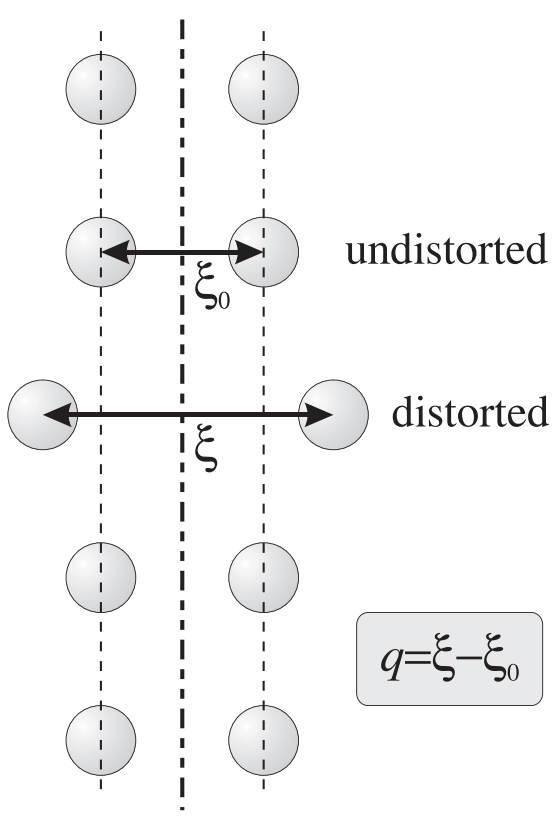

FIG. 1. On the meaning of the coordinate $q$. For details, see the text. Reproduced with permission from C. Merschjann, "Optically generated small polarons: Time-resolved pump-multiprobe experiments in lithium niobate vs. random walk charge transport simulations in oxide crystals," Ph.D. dissertation (Osnabrueck University, 2007). Copyright 2007 Shaker Verlag $\mathrm{GmbH}$, Aachen, Germany.

Real crystals contain intrinsic and/or extrinsic point defects, whose formal charge may differ from that of the regular sites. The energy of a polaron at such a site is additionally decreased by $-E_{\mathrm{C}}$. Due to different ionic environments, the values of the polaronic force constant may slightly differ from those of the above addressed situation, labeled by $F^{\prime}$ instead of $F$. In this case, the polaron energy becomes

$$
E_{\mathrm{min}, \mathrm{D}}=-E_{\mathrm{p}}^{\prime}-E_{\mathrm{C}}=-E_{\mathrm{GP}} .
$$

The resulting state is often referred to as bound small polaron or simply bound polaron, while the former is called

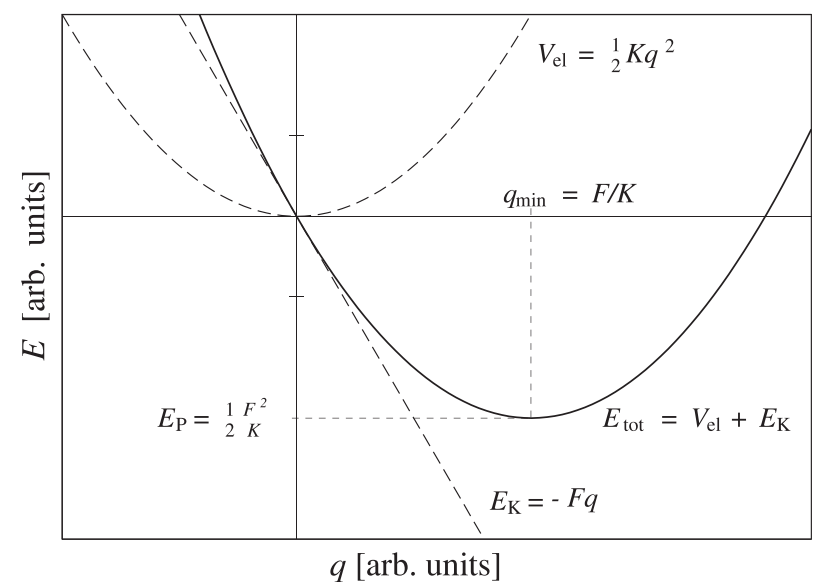

FIG. 2. Sketch of the energies relevant for the small polaron problem. Reproduced with permission from C. Merschjann, "Optically generated small polarons: Time-resolved pump-multiprobe experiments in lithium niobate vs. random walk charge transport simulations in oxide crystals," Ph.D. dissertation (Osnabrueck University, 2007). Copyright 2007 Shaker Verlag $\mathrm{GmbH}$, Aachen, Germany. free polaron with the polaron energy $E_{\mathrm{FP}}=E_{\mathrm{P}}$. The electrons localized in this way can nevertheless move in the crystal lattice, taking the induced lattice distortion with them. At room temperature, the charge transport proceeds via hopping transitions (incoherent motion ${ }^{1,7}$ ).

As to the situation of $\mathrm{LiNbO}_{3}$, Faust et al. found that the conductivity can be well described by the hopping model for temperatures down to $150 \mathrm{~K} .{ }^{57}$ Again, MCM can be applied for a detailed description of the polaronic hopping transport. Consideration of thermally activated transport yields that the thermal activation energy equals half the polaron binding energy

$$
E_{\mathrm{a}}=E_{\mathrm{FP}} / 2 .
$$

We note that it was shown by Austin and Mott that in real ionic crystals the activation energy may be less than $E_{\mathrm{FP}} / 2 .^{54}$

\section{B. Small free and bound polarons in $\mathrm{LiNbO}_{3}$}

To date, three different types of small electron polarons and one type of small hole polaron have unambiguously been identified in $\mathrm{LiNbO}_{3}{ }^{4,57,58}$

It has been proposed in earlier works that the features described here and in the following sections may be explained by electrons located at or in oxygen vacancies, $\mathrm{V}_{\mathrm{O}} \cdot{ }^{11,59}$ However, measurements of the mass density ${ }^{60}$ and ESR results ${ }^{61}$ were highly incommensurate with the oxygenvacancy model, and it was disproved for $\mathrm{LiNbO}_{3}$ (see Sec. 6.2 in Ref. 4, see also Refs. 58 and 62). In addition, another electron quad-polaronic center, the so-called $Q$-polaron, has been proposed in order to describe the high-frequency absorption in reduced $\mathrm{LiNbO}_{3},{ }^{63}$ but has been disproved meanwhile (see Sec. 6.1 in Ref. 4). Therefore, no further comments are given concerning this point.

All types of small polarons result from the intrinsic defect structure of $\mathrm{LiNbO}_{3}$ that-although complex-has been modeled from first principles in very recent articles by Li et $a l .{ }^{64,65} \mathrm{~A}$ sketch of the polarons addressed in this work is given in Fig. 3.

The free small electron polaron (FP) in $\mathrm{LiNbO}_{3}$ consists of an electron that is localized at a regular $\mathrm{Nb}$ site. ${ }^{57} \mathrm{It}$ is represented throughout this work as $\mathrm{Nb}_{\mathrm{Nb}}^{4+}$ or free polaron and is the most shallow electron trap known in $\mathrm{LiNbO}_{3}$. Faust et al. could identify the free polaron only in samples doped with $\mathrm{Mg}$ or $\mathrm{Zn}$ above the optical-damage resistance threshold. ${ }^{57}$ This is due to the fact that in samples below the threshold, deeper traps, $\mathrm{Nb}_{\mathrm{Li}}$, are still present.

Bound small electron polarons $(\mathrm{GP}), \mathrm{Nb}_{\mathrm{Li}}^{4+}$ or simply bound polarons, show properties similar to $\mathrm{Nb}_{\mathrm{Nb}}^{4+}$, except at higher binding and absorption energies. ${ }^{58}$ This type of electron polaron is found in congruently melting $\mathrm{LiNbO}_{3}$, in reduced samples by exposure to visible or ultraviolet light at cryogenic temperatures, or at high temperatures without illumination. ${ }^{58,61,66}$ In both cases, they are metastably created upon optical or thermal dissociation of bipolarons. Another possibility to obtain bound polarons in unreduced crystals is illumination with intense pulsed light. ${ }^{9,67,68}$ Bound polarons further play a decisive role for the photorefractive effect in 

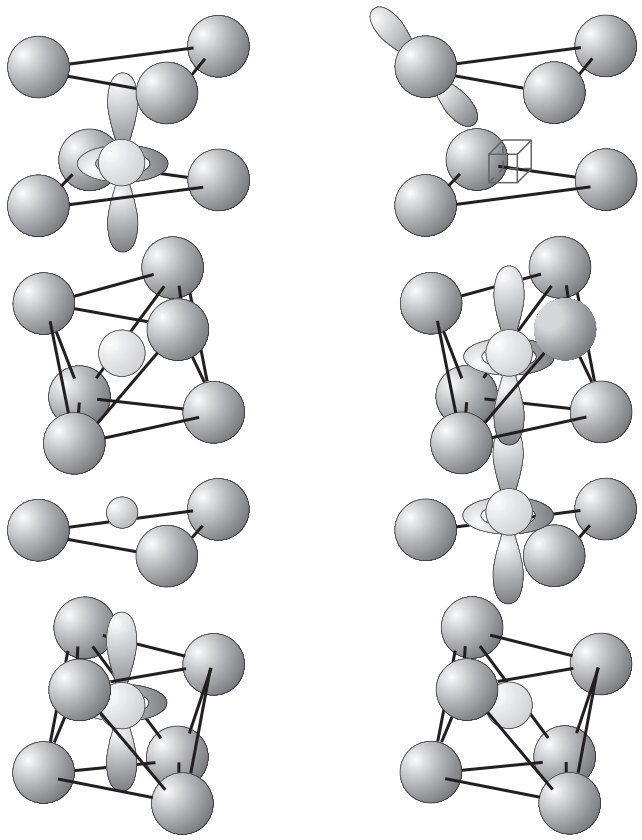

FIG. 3. Structure of $\mathrm{LiNbO}_{3}$ with the intrinsic defects $\mathrm{Nb}_{\mathrm{Li}}$ and $\mathrm{V}_{\mathrm{Li}}$. The related small polarons are depicted using their idealized orbital structure. Lattice distortions are not shown. Reproduced with permission from C. Merschjann, "Optically generated small polarons: Time-resolved pump-multiprobe experiments in lithium niobate vs. random walk charge transport simulations in oxide crystals," Ph.D. dissertation (Osnabrueck University, 2007). Copyright 2007 Shaker Verlag GmbH, Aachen, Germany.

$\mathrm{LiNbO}_{3}$ and $\mathrm{LiNbO}_{3}: \mathrm{Fe}$. They substantially affect the photoconductivity and are in fact the shallow photosensitive level in the two-center model. ${ }^{69}$ Two-color holography is possible utilizing these shallow traps. ${ }^{70}$

The ground state of electrons in reduced congruently melting $\mathrm{LiNbO}_{3}$ is the bound small bipolaron (BP), $\mathrm{Nb}_{\mathrm{Li}}^{4+}: \mathrm{Nb}_{\mathrm{Nb}}^{4+66}$. ${ }^{4}$ the presence of bipolarons, a largely enhanced dark conductivity and photoconductivity is found. ${ }^{71}$ The related activation energies are evaluated and discussed in Ref. 4. Light illumination or elevated temperatures lead to a reversible dissociation of bipolarons in favor of metastable bound $\mathrm{Nb}_{\mathrm{Li}}^{4+}$ polarons. ${ }^{58,61,66}$ While the former mechanism can be applied for two-color holography in reduced undoped $\mathrm{LiNbO}_{3},{ }^{41}$ the latter is mainly responsible for the conductivity in reduced samples. ${ }^{71}$ Deducing the thermal activation energy and the bipolaron binding energy from experimental data is not as straightforward as for single polarons. This is due to the fact that, in contrast to the above given model, this is a two-site bipolaron (Heitler-London bipolaron). ${ }^{72}$ It could, however, be shown that the difference between the binding energy of the bipolaron and that of two bound polarons is about $0.27 \mathrm{eV} .^{66}$

Bound small hole polarons $(\mathrm{HP}), \mathrm{O}^{-}$, are defect electrons located at an oxygen ion in the vicinity of a cation vacancy, most probably $\mathrm{V}_{\mathrm{Li}} .{ }^{5}$ They can be created in $\mathrm{LiNbO}_{3}$ by electron- and X-ray irradiation, ${ }^{73,74}$ as well as by twophoton processes of intense green light. ${ }^{68}$ Since the spatial relation of the Li vacancies among themselves and to $\mathrm{Nb}_{\mathrm{Li}}$ antisite defects is not known, the contribution of hole polarons to the thermally and optically activated charge transport in $\mathrm{LiNbO}_{3}$ is to date still unclear. Therefore, no definite values can be given for the hole polaron binding energy and thermal activation energy.

\section{OPTICAL ABSORPTION FEATURES OF SMALL POLARONS}

\section{A. Modelling}

The optical absorption features of small polarons can be attributed to their optically activated transport. Assuming that the electron is initially at site $1\left(q_{1}\right)$ (cf. Fig. 4), the minimum energies for free polarons are given by

$$
E_{1}=\frac{1}{2} K q^{2}-F q_{1} \quad \text { and } \quad E_{2}=\frac{1}{2} K q^{2}-F q_{2}=0
$$

since $q_{2}=q_{\min , 2}=0$ without the excess electron. For frequencies, $\omega$, exceeding the phonon frequencies $\left(\omega \gg \omega_{\mathrm{LO}}\right)$, the Franck-Condon principle states that the lattice distortion does not change essentially during an electronic transition. Therefore, one has only to consider the electronic energy, with the coordinates of minimum total energy, $q_{\min , 1}=F / K$ and $q_{\min , 2}=0$, unchanged. One then finds the energy for a photon-assisted hopping of free polarons

$$
\begin{aligned}
E_{\mathrm{FP}}^{\mathrm{opt}} & =E_{\min }\left(q_{2} \rightarrow q_{1}\right) \\
& =E_{\mathrm{C}, 2}\left(q_{2}=q_{\mathrm{min}, 2}\right)-E_{\mathrm{C}, 1}\left(q_{1}=q_{\mathrm{min}, 1}\right) \\
& =\frac{F^{2}}{K}=2 E_{\mathrm{FP}} .
\end{aligned}
$$

This energy corresponds to the vertical transition depicted in Fig. 4.

For a bound polaron, the electronic energy at the initial site has the additional perturbation $-E_{\mathrm{C}}$. The energy required for the photon-assisted hopping of bound polarons reads

$$
E_{\mathrm{opt}}^{\mathrm{GP}}=\frac{F^{2}}{K}+E_{\mathrm{C}}=2 E_{\mathrm{FP}}+E_{\mathrm{C}} .
$$

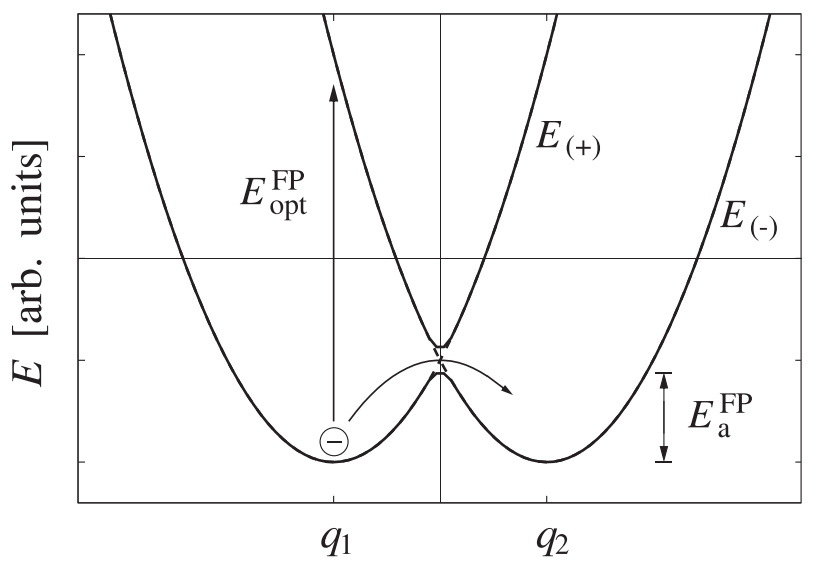

FIG. 4. Optically induced transport of small free polarons. Reproduced with permission from C. Merschjann, "Optically generated small polarons: Timeresolved pump-multiprobe experiments in lithium niobate vs. random walk charge transport simulations in oxide crystals," Ph.D. dissertation (Osnabrueck University, 2007). Copyright 2007 Shaker Verlag GmbH, Aachen, Germany. 
Again, this energy can be ascribed to the vertical transition from initial to final potential that now differ in their minimum energy. The frequency dependence of the AC transport has initially been derived from the MCM by Reik and Heese. ${ }^{75}$ Subsequent authors ${ }^{3,6,7,76}$ found that in the classical limit $\left(2 k_{\mathrm{B}} T>\hbar \omega_{\mathrm{LO}}\right)$, the conductivity related to polarons can be described by

$$
\begin{gathered}
\sigma_{\mathrm{pol}}(\omega, T)=N e \mu_{0}^{\mathrm{na}} \frac{k_{\mathrm{B}} T}{\hbar \omega} \exp \left[-\frac{\left(E_{\mathrm{opt}}^{\mathrm{P}}-\hbar \omega\right)^{2}}{8 E_{\mathrm{FP}}^{*} k_{\mathrm{B}} T}\right], \\
\mu_{0}^{\mathrm{na}}=g \frac{e a^{2}}{k_{\mathrm{B}} T} W_{0}^{\mathrm{na}}, \\
W_{0}^{\mathrm{na}}=\frac{J^{2}}{\hbar}\left(\frac{\pi}{4 E_{\mathrm{a}} k_{\mathrm{B}} T}\right)^{\frac{1}{2}} .
\end{gathered}
$$

Here, $N$ is the number density of polarons, $a$ is the hopping distance, $g$ is a dimensionless number of order 1 , and $\mu_{0}^{\text {na }}$ and $W_{0}^{\text {na }}$ are the nonadiabatic polaron mobility and hopping rate, respectively. The energy $E_{\mathrm{opt}}^{\mathrm{P}}$ has to be replaced by the respective maximum energy for optical transitions of free and bound polarons, $E_{\mathrm{opt}}^{\mathrm{FP}}$ or $E_{\mathrm{opt}}^{\mathrm{GP}}$, and $E_{\mathrm{FP}}^{*}$ is $E_{\mathrm{FP}}$ or $E_{\mathrm{FP}}$. The optical absorption coefficient $\alpha$ is

$$
\alpha_{\mathrm{pol}}(\omega, T)=\frac{1}{n \epsilon_{0} c} \sigma_{\mathrm{pol}}(\omega, T)
$$

with $c$ being the velocity of light, $n$ the refractive index of the material, and $\epsilon_{0}$ the vacuum permittivity. The resulting absorption band resembles a Gaussian curve, with a prefactor $1 / \omega$, centered at $\omega_{\max }=E_{\mathrm{opt}}^{\mathrm{P}} / \hbar$, with a full width at half maximum

$$
\omega_{\mathrm{FWHM}} \approx 4 \sqrt{2 \ln [2] E_{\mathrm{FP}}^{*} k_{\mathrm{B}} T}
$$

that is more pronounced at lower frequencies $\left(\omega<\omega_{\max }\right)$.

\section{B. Experimental fingerprints}

Comparisons with experimental data, e.g., for free polarons in $\mathrm{LiNbO}_{3},{ }^{57}$ show that the theory presented above is probably oversimplified. One possible reason may be that here only transitions from the electronic ground state at the initial site to the ground state at the final site are considered. ${ }^{5}$ The orbital structure of polarons including their bound excited states as well as excitation to states with ballistic transport is neglected.

The absorption spectra of the individual electron and hole polarons in $\mathrm{LiNbO}_{3}$ are modeled by means of the spectral dependence of the respective absorption cross section in Fig. 5 according to Ref. 77.

The free polaron is characterized by a broad optical absorption band in the near infrared spectral range, centered at about $E_{\mathrm{opt}}^{\mathrm{FP}}=1.0 \mathrm{eV}(\hat{=} 1280 \mathrm{~nm})$-besides showing typical ESR spectra. ${ }^{57,78}$ At the same time, measurements of the electrical conductivity yield an activation energy for phonon-assisted hopping transport, $E_{\mathrm{a}}^{\mathrm{FP}}=0.2-0.3 \mathrm{eV}$. These values are in line with the theory for the free small polaron; the corresponding polaron binding energy is

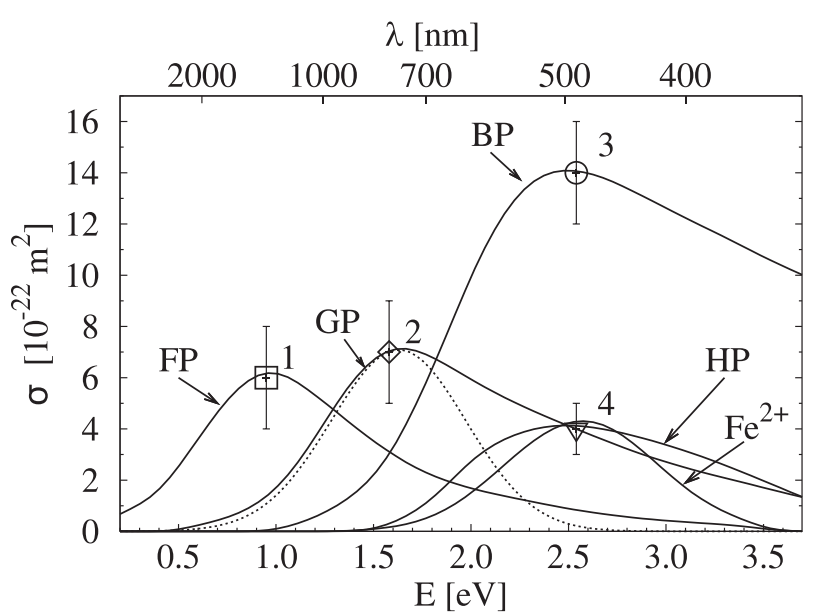

FIG. 5. Spectral dependence of the absorption cross section of small free (FP) and bound (GP) electron polarons, of bipolarons (BP), and of small hole polarons (HP). The spectral fingerprint of $\mathrm{Fe}_{\mathrm{Li}}^{2+}$ that can be described by small polaron theory, as well, is depicted for reasons of comparison. Note that the absorption cross section of BP is about two times higher than those of the single polarons because the oscillator strength is proportional to the number of electrons in an absorbing system. Reproduced with permission from Merschjann et al., J. Phys. Condens. Matter 21, 015906 (2009). Copyright 2009 IOP Publishing.

$E_{\mathrm{FP}}=0.5 \mathrm{eV} .^{57}$ However, detailed investigations of the absorption spectra indicate a slight additional binding of about $0.1 \mathrm{eV}$, influencing the optical absorption at low temperature. ${ }^{57}$ One possible reason could be a location of $\mathrm{Nb}_{\mathrm{Nb}}^{4+}$ in the vicinity of $\mathrm{a} \mathrm{Mg}^{2+}$ (or respective impurity) ion on $\mathrm{Li}^{+}$ site. ${ }^{62}$ The electronic charge transport in $\mathrm{LiNbO}_{3}$ proceeds via $\mathrm{Nb}^{4+} .{ }^{62}$ There is strong evidence that these $\mathrm{Nb}^{4+}$ ions are mainly existing in form of free polarons, and that the transport is essentially of thermally activated hopping type. ${ }^{18,62}$ Bound polarons are accompanied by an absorption band centered at $E_{\mathrm{opt}}^{\mathrm{GP}}=1.6 \mathrm{eV}(\hat{=} 760 \mathrm{~nm})$, which implies a polaron binding energy $E_{\mathrm{FP}}^{\prime} \approx 0.55 \mathrm{eV}$ and the additional term $W_{\mathrm{D}} \approx 0.5 \mathrm{eV} .^{58,67}$ The thermal activation energy may be estimated to be $E_{\mathrm{a}}^{\mathrm{GP}} \approx 0.65 \mathrm{eV}$. Bipolarons feature a broad absorption band, which is centered at $E_{\mathrm{opt}}^{\mathrm{BP}}=2.5 \mathrm{eV}$ ( $\hat{=} 500 \mathrm{~nm}$ ) and covers the whole visible spectral range, giving the samples a grayish color. ${ }^{61}$ The BP absorption cross section is about two times higher than those of the single polarons because the oscillator strength is proportional to the number of electrons in an absorbing system. The hole polaron exhibits a broad absorption band in the visible range, centered at $E_{\mathrm{opt} .}^{\mathrm{HP}} \approx 2.5 \mathrm{eV}(\hat{=} 500 \mathrm{~nm}) .{ }^{73}$ A detailed ESR and optical investigation of the hole-polaron absorption is hindered by the band of $\mathrm{Nb}_{\mathrm{Li}}^{4+}$, which always appeared in the cited works. The optical absorption of $\mathrm{O}^{-}$is described by a photon-assisted hopping of the hole between equivalent adjacent $\mathrm{O}^{2-}$ ions, which are located around the Li vacancy, thus restricting the hole polaron to one single $\mathrm{V}_{\mathrm{Li}}{ }^{5}$

\section{OPTICAL GENERATION OF SMALL POLARONS}

The description of the transmission loss of an ultra-short laser pulse propagation through a LN crystal is based on the model considering various types of non-instantaneous responses in LN depicted in Fig. 6. 


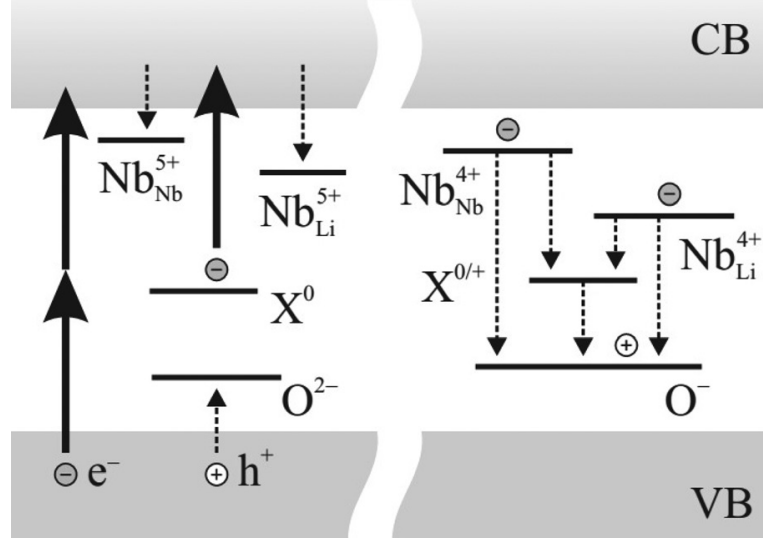

FIG. 6. Generation and relaxation of small polarons in nominally undoped $\mathrm{LiNbO}_{3}$. Left: The driving process of polaron formation is band-to-band excitation, resulting in electron-hole pairs. According to the band gap of $3.8 \mathrm{eV}$, two-photon absorption dominates this process in the visible and near-infrared spectral range. Carrier localization results in the formation of small free $\mathrm{Nb}_{\mathrm{Nb}}^{4+}$, small bound $\mathrm{Nb}_{\mathrm{Li}}^{4+}$, and small $\mathrm{O}^{-}$hole polarons. At low intensities, the contribution of carriers excited via single-photon absorption from extrinsic defect centers $\mathrm{X}^{0}$ may be considered until these are depopulated. Right: Recombination paths are imprinted by electron-hole recombination of FP and GP with HP. The recharging of the extrinsic $\mathrm{X}^{0 /+}$ center may represent an intermediate step depending on the transfer probabilities. Reproduced with permission from C. Merschjann, "Optically generated small polarons: Time-resolved pump-multiprobe experiments in lithium niobate vs. random walk charge transport simulations in oxide crystals," $\mathrm{Ph}$.D. dissertation (Osnabrueck University, 2007). Copyright 2007 Shaker Verlag $\mathrm{GmbH}$, Aachen, Germany.

Generation of small polarons can be performed by optical means by means of one-photon and two-photon absorption. The most prominent generation paths are summarized in the following and are depicted in Figs. 6 and 7. It is noteworthy to state that optically generated small polarons always represent non-equilibrium states-contrary to thermally stable small polarons, such as, e.g., by thermal pre-treatment that enhances the presence of carriers in the

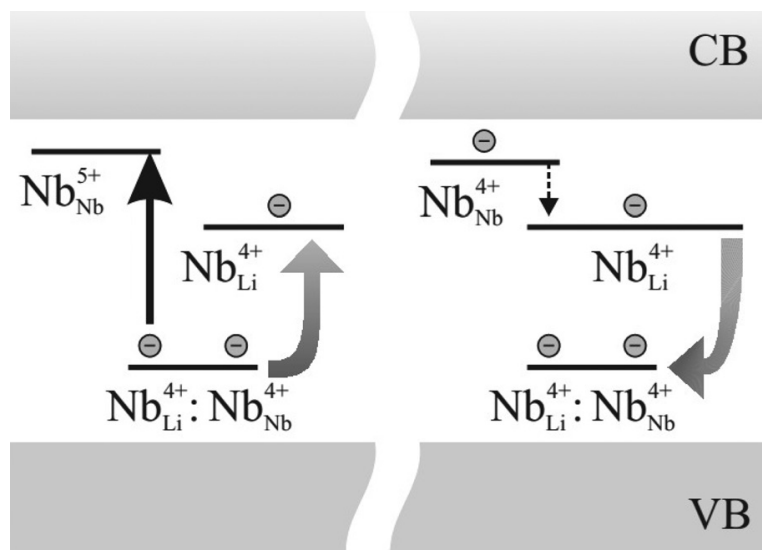

FIG. 7. Generation and relaxation of small polarons in thermally reduced $\mathrm{LiNbO}_{3}$. Left: The $\mathrm{Nb}_{\mathrm{Li}}^{4+}: \mathrm{Nb}_{\mathrm{Nb}}^{4+}$ bipolaron is optically dissociated, and, thereby, a small free $\mathrm{Nb}_{\mathrm{Nb}}^{4+}$ polaron and a bound $\mathrm{Nb}_{\mathrm{Li}}^{4+}$ polaron are formed. Right: By incoherent hopping of both FP and BP, the two small polarons recombine to a bipolaron. Reproduced with permission from C. Merschjann, "Optically generated small polarons: Time-resolved pump-multiprobe experiments in lithium niobate vs. random walk charge transport simulations in oxide crystals," Ph.D. dissertation (Osnabrueck University, 2007). Copyright 2007 Shaker Verlag GmbH, Aachen, Germany. crystal bulk. Thus, optically generated small polarons vanish after a specific small polaron lifetime defining the loss of the localized carrier, i.e., they are metastable.

For generation, the presence of charge carriers, holes or electrons, is inevitable, which-in principle-can be performed in nominally undoped $\mathrm{LiNbO}_{3}$ via one-photon absorption from the valence to the conduction band (band-to-band transition). However, due to the large band gap of $3.8 \mathrm{eV}$, this type of small polaron generation requires ultraviolet light $(<325 \mathrm{~nm})$ available from excimer lasers. In this case, small polaron generation and interband photorefraction may superimpose. $^{79,80}$

More prominent is the mechanism depicted in Fig. 6 first described by Schirmer and von der Linde ${ }^{68}$ for the generation of small bound polarons. Accordingly, electrons are excited from the valence band to the conduction band by a twophoton absorption (TPA) process. They are then trapped at $\mathrm{Nb}_{\mathrm{Li}}$ antisite defects to form small bound $\mathrm{Nb}_{\mathrm{Li}}^{4+}$ polarons. The remaining holes in the valence band become localized as $\mathrm{O}^{-}$ hole polarons in the vicinity of lithium vacancies. ${ }^{5,58,68}$ Upon their recombination, the light-induced absorption changes totally vanish in the dark.

The formation time of small bound polarons has been systematically studied by Sasamoto et al. ${ }^{17}$ using femtosecond pump-probe spectroscopy and nominally undoped $\mathrm{LiNbO}_{3}$. Pulses with durations of $80 \mathrm{fs}$ at a wavelength of $400 \mathrm{~nm}$ allowed for the statement that small bound polarons are formed within a timescale of $100 \mathrm{fs}$, that is, consistent with the results of other authors. ${ }^{13,37,81-83}$ For details, see experimental section (Section IV B) below.

A similar model and study related to small free $\mathrm{Nb}_{\mathrm{Nb}}^{4+}$ polaron formation was introduced by Qiu et al. ${ }^{16}$ Accordingly, electrons excited via two-photon absorption are first generated (hot carriers); then, they relax to the lower edge of the conduction band by energy dissipation to the lattice (thermalization) where small free $\mathrm{Nb}_{\mathrm{Nb}}^{4+}$ polarons (and/or $\mathrm{Nb}_{\mathrm{Li}}^{4+}$ bound polarons in crystals grown from the congruently melting composition) are generated. The transfer to $\mathrm{Nb}_{\mathrm{Li}}^{4+}$ bound polarons may also occur upon thermally activated transport via $\mathrm{Nb}_{\mathrm{Nb}}$ centers. It is noteworthy that two-photon absorption overlaps the possible transient signals of hot carriers, and thus may not be observed experimentally in $\mathrm{LiNbO}_{3}{ }^{82}$ Nevertheless, the validity of these models has been studied by femtosecond experiments in several groups in the last decade yielding several important measures. For instance, the efficiency of the carrier generation by TPA is well determined using laser pulses with duration of 80 fs up to $10 \mathrm{~ns}$ (values of $\beta \approx(1.5-4) \mathrm{GW} / \mathrm{mm}$ at $532 \mathrm{~nm}$ are reported and summarized in Section IV B).

The optical generation of small polarons in nominally pure, thermally reduced $\mathrm{LiNbO}_{3}$ has been addressed by Jermann et al. ${ }^{69}$ They found that $\mathrm{Nb}_{\mathrm{Li}}^{4+}: \mathrm{Nb}_{\mathrm{Nb}}^{4+}$ bipolarons are dissociated by green light such that $\mathrm{Nb}_{\mathrm{Li}}^{4+}$ bound polarons are formed (cf. Fig. 7). In the dark, the bound polarons recombine and form bipolarons again. ${ }^{69}$ At that time, the role of $\mathrm{Nb}_{\mathrm{Nb}}^{4+}$ free polarons was not analyzed. ${ }^{69}$ In this process, it is very much likely that the electron localized at the regular $\mathrm{Nb}_{\mathrm{Nb}}$ site is optically transferred to a next-neighboring $\mathrm{Nb}_{\mathrm{Nb}}$ site, i.e., the small bound GP remains at its position. 
Merschjann et al. were able to deconvolve the appearance and recombination dynamics of free small $\mathrm{Nb}_{\mathrm{Nb}}^{4+}$ polarons upon bipolaron gating in thermally reduced $\mathrm{LiNbO}_{3}$ by means of time-resolved spectroscopy and ns-laser pulses at $2.5 \mathrm{eV}{ }^{15}$ Its formation time was estimated to values below 100 fs using fs-spectroscopy; ${ }^{16,84}$ an onset time of about 80 fs prior to small polaron formation and attributed to the cooling of hot electrons has been verified by Badorreck et al. ${ }^{84}$

\section{A. Light-induced small polaron absorption}

\section{Fundamental description}

The formation of small polarons and their subsequent dynamical behavior upon light exposure is reflected by the build-up of a transient absorption, i.e., a small polaron absorption that is proportional to the product of number density and absorption cross section (cf. Fig. 5). This type of optically induced transient absorption often is called lightinduced absorption. The total absorption of a nominally undoped $\mathrm{LiNbO}_{3}$ at a specific optical frequency thus is composed of a fundamental absorption $\alpha_{0}(\omega)$, and the sum of the absorptions of all types of small polarons, $\alpha_{i}(\omega)$. The fundamental absorption stems from electronic transitions between valence band and conduction band. One assumes here that $\alpha_{0}(\omega)$ does not depend on the experimental conditions (time, temperature, illumination, etc.), but solely on the light frequency $\omega$. On the other hand, the absorption of the different polaronic centers involved may well depend on time, temperature, and illumination features (photon energy, intensity, pulse duration, light polarization)

$$
\alpha(\omega, t)=\alpha_{0}(\omega)+\sum_{i} \alpha_{i}(\omega, t) .
$$

Note that the dependencies of $\alpha_{i}$ on $I$ is not explicitly given in this formula. However, experimental results will show that these dependencies are of crucial importance and have been omitted here only for reasons of clarity.

The absorption by photosensitive centers is the product of the respective absorption cross sections $\sigma_{i}(\omega)$ and their time-dependent number densities $N_{i}(t)$

$$
\alpha_{i}(\omega, t)=N_{i}(t) \sigma_{i}(\omega)
$$

One may split $N_{i}(t)$ into a time-independent and a timedependent part. This leads to

$$
\begin{aligned}
\alpha_{i}(\omega, t) & =\left[N_{0, i}+N_{\mathrm{li}, i}(t)\right] \sigma_{i}(\omega) \\
& =\alpha_{0, i}(\omega)+\alpha_{\mathrm{li}}(\omega, t),
\end{aligned}
$$

where $\alpha_{\mathrm{li}}(\omega, t)$ is the time-dependent light-induced absorption change (or simply light-induced absorption) for the respective center. By combining these equations, one obtains the formula for the time-dependent total absorption, namely

$$
\begin{aligned}
\alpha(\omega, t) & =\alpha_{0}(\omega)+\sum_{i} \alpha_{0, i}(\omega)+\sum_{i} \alpha_{\mathrm{li}, i}(\omega, t) \\
& =\alpha(\omega)+\alpha_{\mathrm{li}}(\omega, t)
\end{aligned}
$$

Thus, the total absorption of the crystal can be described as a sum of the steady-state absorption $\alpha(\omega)$ and the light- induced absorption $\alpha_{\mathrm{li}}(\omega, t)$. The latter is of utmost importance for the study or impact of small polarons in $\mathrm{LiNbO}_{3}$.

\section{Thermally activated decay}

According to the two-center model, ${ }^{12}$ the relaxation of light-induced absorption changes should follow a monoexponential law; i.e., the decay rate is directly proportional to the number density of metastable centers. However, timeresolved measurements of $\alpha_{\mathrm{li}}(t)$ in $\mathrm{LiNbO}_{3}$ show that this is not the case at all. 9,47,67,69 The observed decay spectra are in general best described by an empirical law of stretched exponential functions, named after Kohlrausch, Williams, and Watts. ${ }^{85-87}$ This so-called KWW function is given by

$$
\alpha_{\mathrm{li}}(t)=\alpha_{\mathrm{li}}^{(0)} \cdot \exp \left[-(t / \tau)^{\beta}\right]
$$

with the characteristic absorption $\alpha_{\mathrm{li}}^{(0)}$, the characteristic lifetime $\tau$, and the stretching factor $0 \leq \beta \leq 1$, where $\beta \equiv 1$ denotes the case of a mono-exponential relaxation. Also sums of KWW functions are used to describe the decay of $\alpha_{\mathrm{li}}(t)$ :

$$
\alpha_{\mathrm{li}}(t)=\sum_{i=1}^{n} \alpha_{\mathrm{li}}^{(i)} \cdot \exp \left[-(t / \tau)^{\beta}\right] .
$$

It should be noted, here, that in most cases, the parameter $\alpha_{\mathrm{li}}^{(0)}$ is not identical with the experimentally determined amplitude of the light-induced absorption $\alpha_{\mathrm{li}}^{\max }=\alpha_{\mathrm{li}}(\omega, t=0)$ as a result of limited temporal resolution in ns-pump-probe experiments (see also Ref. 67). Furthermore, the characteristic lifetime $\tau$ does not represent one single lifetime of the metastable state. It is rather to be considered as a mean value of many different lifetimes; thus, Eq. (18) may be seen as a superposition of an infinite number of different monoexponential decays with a certain distribution of lifetimes.

While a general answer to the underlying physics of stretched relaxation dynamics in $\mathrm{LiNbO}_{3}$ could not yet be given, some approaches do indeed exist. Berben et al. were the first to apply Eq. (18) to the decay of $\alpha_{\mathrm{li}}(t)$ in $\mathrm{LiNbO}_{3}: \mathrm{Fe}^{9}$ They suggested a distance-dependent charge transport in such a way that charge carriers take the distance between the initial shallow center and the final deep trap in one hopping transition. According to this model, the probability for the jump should depend on the distance $r$ between initial and final site, obeying a power law, $p \propto r^{-x}$, where $x$ is an empirical parameter. Numerical simulations ${ }^{9}$ showed that the resulting decay curves may well be described by Eq. (18). However, the parameter $x$ could not be deduced from first principles. ${ }^{9}$ Later, Wevering et al. found a mathematical description, based on the aforementioned idea, that lead indeed to a stretched exponential function, namely, $\alpha_{\mathrm{li}}(t)$ $\propto \exp \left[-(t / \tau)^{k / 3}\right]$, where $k$ is an integer. ${ }^{47}$ Nevertheless, the values for $k$ have to be chosen empirically, without knowing their physical meaning. Another approach has been given by Sturman et al. ${ }^{88}$ This model is based on a random walk of nearest-neighbor hopping transitions. After several hopping processes, the carrier finally recombines with a trap. Thereby, a stretched-exponential relaxation is not obtained 
directly. However, if the distances between nearest neighbors are randomly distributed, like, e.g., in an amorphous material, a numerical simulation yields decay curves that are well described by Eq. (18). ${ }^{88}$ It turns out that this feature depends essentially on the distribution of distances, where the longest transition distance mainly determines the lifetime $(p \propto \exp [-r])$. Applied to $\mathrm{LiNbO}_{3}, r$ describes the distance between initial and final site of the optically induced transition.

An important step towards a more microscopic understanding of the underlying transport processes was published by Carnicero et al. ${ }^{18}$ using Monte Carlo simulations. Besides deducing the average, unidirectional transport length in the order of several nanometers (depending on stoichiometry and pump beam intensity, see below), the presence of the site correlation effect was introduced. This effect is based on the observation that an optically generated charge carrier, localized as small polaron at a specific lattice site, has a high probability to be captured by its original trap center, i.e., the center where it was excited from. Based on these findings, Merschjann et al. continued with systematic studies considering a broad spectrum of experimental studies. ${ }^{89}$ In Ref. 19, the effect of trap saturation is taken into account additionally, in order to derive an extended microscopic model for the non-exponential relaxation. Thereby, it is shown that the relaxation dynamics can be successfully reconstructed without consideration of a structural or energetic disorder. Furthermore, the access to particular microscopic measures such as the lifetime of single hopping events and localized-carrier densities is enabled. A further theoretical approach introduced by Guilbert et al. is in preparation; ${ }^{90}$ it is the aim to represent a powerful numerical tool for the modelling of relaxation dynamics for a wide spectrum of experimental parameters, including temperature. Thus, more detailed insight into this complex research field is to be expected.

\section{B. Experimental examples}

Figure 8 shows photographs of two single crystals of $\mathrm{LiNbO}_{3}$ grown from a congruent nominally undoped melt via Czochralski growth technique (Crystal Technology, Inc.) as typically prepared for optical studies (e.g., the surface is polished to optical quality). The left sample is as-grown, while the right sample is thermally pre-treated. Thus, it features a grayish coloration due to the presence of $\mathrm{Nb}_{\mathrm{Li}}^{4+}: \mathrm{Nb}_{\mathrm{Nb}}^{4+}$

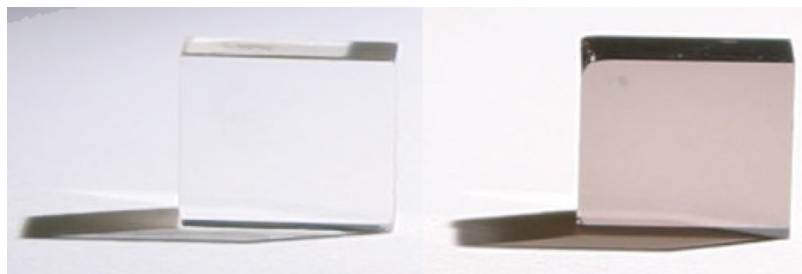

FIG. 8. Photographs of nominally undoped (left) and thermally reduced (right) $\mathrm{LiNbO}_{3}$, as used for most of the optical studies in this section and Section VI. The pre-treated sample was kept at elevated temperature of $(970 \pm 10) \mathrm{K}$ at a reduced pressure of $p<10^{-4}$ mbar for a duration of $6 \mathrm{~h}$. bipolarons and small bound $\mathrm{Nb}_{\mathrm{Li}}^{4+}$ polarons $(\mathrm{GP}),{ }^{19,20}$ as a result of chemical reduction.

\section{Number densities of optically generated small polarons}

The nonlinear optical properties of optically generated small polarons are proportional to their number density $N$, which is strongly dependent on the intensity of the incoming laser pulse. An optical measure for $N$ is the light-induced absorption $\alpha_{\mathrm{li}}^{\max }$ being proportional to $N$ in product with the absorption cross sections. Its dependence as a function of pump beam intensity is depicted in Fig. 9 for nominally undoped (upper) and thermally reduced $\mathrm{LiNbO}_{3}$. The data were collected using a setup for time-resolved pump-multiprobe spectroscopy, described in Refs. 15 and 91. Here, intensities of the pump light $I_{\mathrm{p}} \leq 760 \mathrm{GW} / \mathrm{m}^{2}$ at a wavelength of $\lambda=532 \mathrm{~nm}$ were used.

The initial amplitudes are strongly dependent on the pump beam intensity and reflect number densities of small polarons in the order of $N \approx 10^{23} \mathrm{~m}^{-3}$ considering the absorption cross sections of Fig. 5. The data are given for probe wavelengths of 488, 785, and $1310 \mathrm{~nm}$, thus being close to the maxima of the small polaron absorption features of hole and bi-polarons, of bound bolarons and of free polarons. Remarkably, $\alpha_{\mathrm{li}}^{\max }$ grows quadratically as a function of intensity in the nominally undoped sample that reflects the underlying two-photon absorption process schematically sketched in Fig. 6. In contrast, for thermally reduced $\mathrm{LiNbO}_{3}, \alpha_{\mathrm{li}}^{\max }\left(I_{\mathrm{p}}\right)$ saturates at $\approx 350 \mathrm{GW} / \mathrm{m}^{2}$ for all three

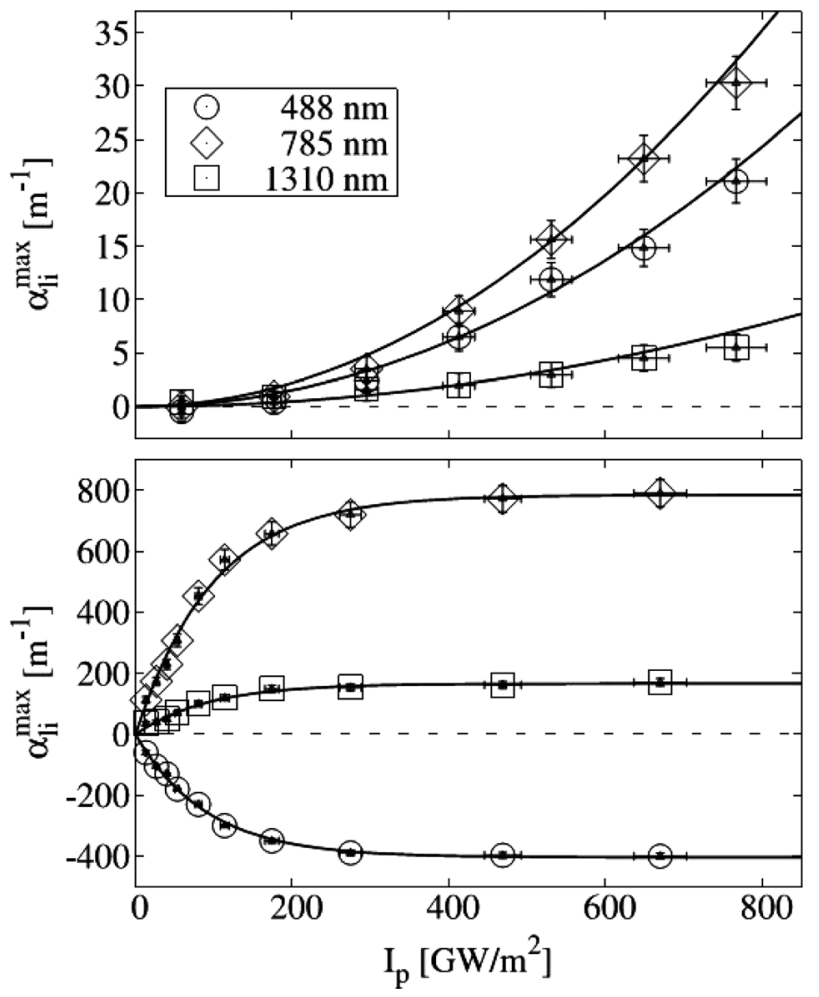

FIG. 9. Intensity dependency of the maximum optically induced absorption due to small polarons in nominally undoped (upper) and thermally reduced $\mathrm{LiNbO}_{3}$ (lower) for the three probe wavelengths 488, 785, and $1310 \mathrm{~nm}$ at room temperature. Reproduced with permission from Merschjann et al., Phys. Rev. B 76, 085114 (2007). Copyright 2007 APS Publishing. 
wavelengths, however, with negative sign for a probe wavelength of $488 \mathrm{~nm}$. This observation can be assigned to the process of bipolaron dissociation: positive values at 785 and $1310 \mathrm{~nm}$ refer to the generation of small bound and free polarons, while the negative values at $488 \mathrm{~nm}$ are due to the optical dissociation of bipolarons. Furthermore, as the number density of BP is limited, the number density of generated small polarons must saturate. In particular, the number density of generated small polarons must be equivalent to the number density of dissociated bipolarons. Taking not only the differences in the absorption cross sections into account but also the spectral overlap of the particular small polaron features at the probing wavelength, it is possible to deduce the number densities of the generated small polarons, ${ }^{77}$ as depicted in Table I.

The efficiency of the two-photon absorption process thus determines the number density of excited carriers, and subsequent formation of small polarons; its measure is the two-photon absorption coefficient $\beta$ that can be obtained experimentally by means of pump beam transmittance as a function of pump beam intensity or via $z$-scan technique. ${ }^{92}$ The values of $\beta$ typically are in the order of a few $\mathrm{cm} / \mathrm{GW}$, so that it is reasonable to use fs- or ps-laser pulses enabling sufficient peak intensities for experimental studies. In order to suppress the influence of intrinsic defects that are accompanied with a shift of the band edge ${ }^{93}$ as well as with absorption features of small bound polarons, Mg-doped, stoichiometric samples are applied by Sasamoto et al. ${ }^{17}$ Fig. 10 shows the results for both ordinary and extra-ordinary light polarization and the use of 80 fs laser pulses at $400 \mathrm{~nm}$. The experimentally determined values of $\beta$ from different authors, congruent crystals, and pulse durations from femtoto nano-seconds are summarized in Table II. All data refer to extraordinary light polarization; the values for ordinary light polarization are slightly smaller in the order of $10 \%$.

We note that $\beta$ is strongly dependent on $\lambda$ and grows as a function of photon energy as it is shown, e.g., in the study of Beyer et al. ${ }^{37}$ for the visible spectral range (400-800 nm).

\section{Recombination dynamics of optically generated small polarons}

Fig. 11 shows a typical temporal decay behavior of the small-polaron based light-induced absorption in the dark, i.e., upon a short ns-laser pulse at a wavelength of $532 \mathrm{~nm}$, exemplarily for thermally reduced $\mathrm{LiNbO}_{3}$ (cf. right sample

TABLE I. Estimated number densities (saturation, ns-pulse experiments) and absorption cross sections for the different kinds of photochromic centers. For bipolarons, the number density in the dark is given. Reproduced with permission from Merschjann et al., J. Phys. Condens. Matter 21, 015906 (2009). Copyright 2009 IOP Publishing.

\begin{tabular}{lccccc}
\hline \hline & $N\left(10^{22} \mathrm{~m}^{-3}\right)$ & $N / N_{\text {sites }}$ & $\sigma\left(10^{-22}\right) \mathrm{m}^{2}$ & $\lambda(\mathrm{nm})$ & Ref. \\
\hline $\mathrm{FP}$ & $30 \pm 5$ & $1.5 \times 10^{-5}$ & $6 \pm 2$ & 1310 & $\ldots$ \\
$\mathrm{GP}$ & $12 \pm 3$ & $6.0 \times 10^{-4}$ & $7 \pm 2$ & 785 & 14,22, and 23 \\
$\mathrm{BP}$ & $70 \pm 8$ & $3.5 \times 10^{-3}$ & $14 \pm 2$ & 488 & $\ldots$ \\
$\mathrm{HP}$ & $30 \pm 5$ & $3.8 \times 10^{-4}$ & $4 \pm 1$ & 488 & $\ldots$ \\
$\mathrm{Fe}_{\mathrm{Li}}^{2+}$ & $2 \pm 1$ & $\ldots$ & $4.5 \pm 0.8$ & 488 & 20 and 31 \\
\hline \hline
\end{tabular}

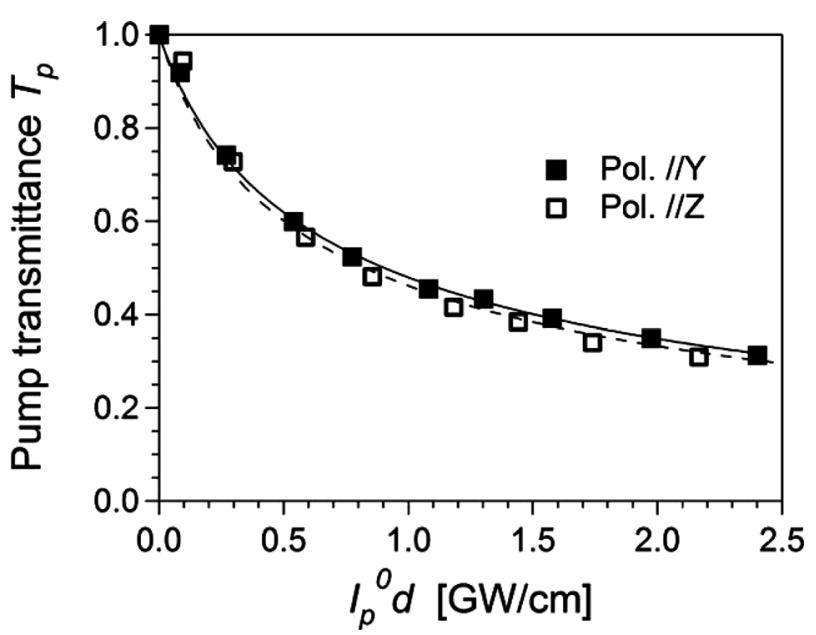

FIG. 10. Pump transmittance $T_{\mathrm{p}}$ measured for $\mathrm{Mg}$-doped, stoichiometric $\mathrm{LiNbO}_{3}$ as a function of the product of pump beam intensity $I_{\mathrm{p}}^{0}$ and sample thickness $d$. Solid and dashed lines indicate theoretical fitting curves for ordinary light polarization ( $\| \mathrm{Y}$ ) and extraordinary light polarization (\| Z), respectively. Reproduced with permission from J. Appl. Phys. 105, 083102 (2009). Copyright 2009 AIP Publishing LLC.

in Fig. 8). The dominating process is the optical dissociation of $\mathrm{Nb}_{\mathrm{Li}}^{4+}: \mathrm{Nb}_{\mathrm{Nb}}^{4+}$-bipolarons as depicted in Fig. 7 (left half), i.e., small free $\mathrm{Nb}_{\mathrm{Nb}}^{4+}$ and bound $\mathrm{Nb}_{\mathrm{Li}}^{4+}$ polarons are generated. The plot of Fig. 11 covers a regime of 9 time decades, from nanoseconds to seconds, whereby-for all of the three probe wavelengths close to the maximum absorption features of FP $(1310 \mathrm{~nm})$, of GP $(785 \mathrm{~nm})$, and of HP as well as BP $(488 \mathrm{~nm})$ - a similar decay behavior with a characteristic decay time in the order of several milliseconds is found $\left(\tau_{\mathrm{FP}}=(2 \pm 1) \times 10^{-3} \mathrm{~s}, \tau_{\mathrm{GP}}=(4 \pm 2) \times 10^{-3} \mathrm{~s}\right.$, and $\tau_{\mathrm{BP}}=(3 \pm 1)$ $\left.\times 10^{-3} \mathrm{~s}\right)$. As there is no difference between the signal dynamics at $1310 \mathrm{~nm}$ and $785 \mathrm{~nm}$, despite a difference in the starting amplitude $\alpha_{\mathrm{li}}^{0}$, it must be concluded that the measurement is not sensitive to the presence of $\mathrm{Nb}_{\mathrm{Nb}}^{4+}$ polarons, maybe as a result of limited sensitivity in the time regime up to $\mu \mathrm{s}$ and/or the strong signal at $785 \mathrm{~nm}$. According to Fig. 5 , there is a distinct amplitude of the GP absorption feature at $1310 \mathrm{~nm}$, such that both signals are due to the time decay of GP. The negative starting amplitude at $488 \mathrm{~nm}$ inevitably points to the optical dissociation of BP that is already terminated in the beginning of the temporal spectrum; i.e., the dissociation time cannot be deduced from this measurement and is expected to be in the sub-ps range, as demonstrated in

TABLE II. Experimentally determined values of $\beta$ for various crystals and pulse durations.

\begin{tabular}{lcccc}
\hline \hline Crystal & $\begin{array}{c}\text { Wavelength } \\
(\mathrm{nm})\end{array}$ & $\begin{array}{c}\text { Pulse } \\
\text { duration (fs) }\end{array}$ & $\beta\left[10^{-12}(\mathrm{~m} / \mathrm{W})\right]$ & Ref. \\
\hline stoichiometric: & & & & \\
Mg-doped LiNbO & & & & \\
congruent: & 400 & 240 & $\approx 3.5$ & 17 \\
$\mathrm{LiNbO}_{3}$ & $\approx 480$ & 7.000 & 1.5 & 97 \\
$\mathrm{LiNbO}_{3}$ & 528.8 & 25.000 & 2.5 & 95 \\
$\mathrm{LiNbO}_{3}$ & 532 & 30.000 & 3.8 & 96 \\
$\mathrm{LiNbO}_{3}$ & 532 & 55.000 & 2.1 & 97 \\
$\mathrm{LiNbO}_{3}$ & 532 & & & \\
\hline \hline
\end{tabular}




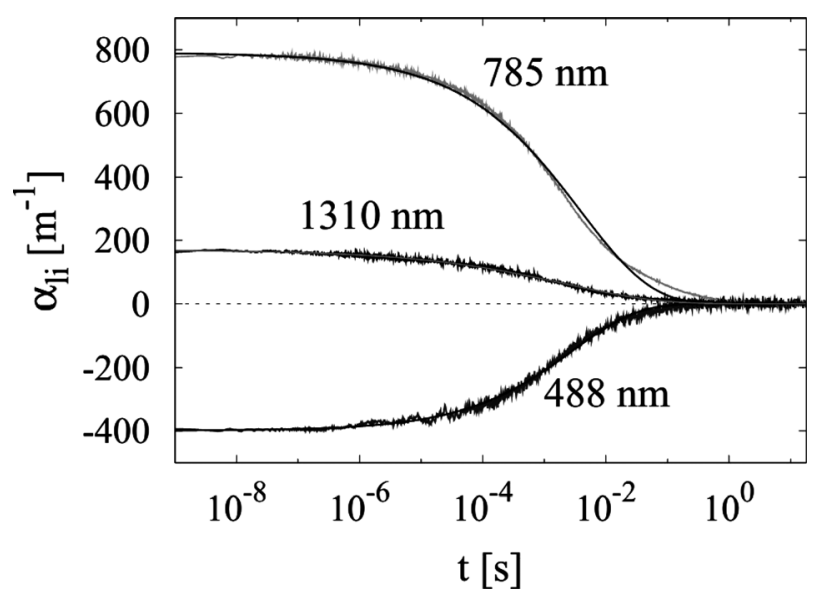

FIG. 11. Temporal dynamics of the light induced absorption and transparency in a thermally reduced sample of $\mathrm{LiNbO}_{3}$ upon exposure with a single ns-laser pulse $\left(\tau_{\mathrm{p}}=8 \mathrm{~ns}, \lambda_{p}=532 \mathrm{~nm}\right.$, intensity $\left.I_{\mathrm{p}}=670 \mathrm{GW} / \mathrm{m}^{2}, \mathbf{e} \perp c\right)$. Transient absorption is found at 785 and $1310 \mathrm{~nm}$ due to the formation of small bound polarons while transient transparency occurs at $488 \mathrm{~nm}$ that is assigned to the optical dissociation of BP. Besides, the decay dynamics reveal a mean lifetime in the millisecond regime, for all wavelengths. Reproduced with permission from Merschjann et al., Phys. Rev. B 76, 085114 (2007). Copyright 2007 APS Publishing.

Section IVB3. Nevertheless, the recombination time can be determined and coincides within the error bars with the GP decay time. This is reasonable according to Fig. 7. In all these spectra, the appearance of $\mathrm{O}^{-}$hole polarons is not found. The shape of all temporal dynamics is well described by stretched exponential functions according to Eq. (18) (solid lines in Fig. 11).

\section{Optical formation time of small polarons}

The small polaron formation time has been addressed in literature by a few authors, already. ${ }^{13,16,17,37,81-83}$ As the formation time is expected in the sub-ps-time regime, studies based on ultrafast spectroscopy with sufficiently small pulse durations, i.e., $\ll 1$ ps, and large pulse energies appropriate for the small absorption features $(>1 \mathrm{~mJ})$ are required inevitably. The first experimental data on the interaction of fs-laser pulses with nominally undoped $\mathrm{LiNbO}_{3}$ have been published by the research group of Williams. ${ }^{81}$ Based on systematic studies, it is meanwhile possible to assign the complex transient signals to specific small polaron species, as summarized in the following.

The formation time of small free $\mathrm{Nb}_{\mathrm{Nb}}^{4+}$ polarons at room temperature has been determined by Qiu et al. in Ref. 16 to 110 fs. Here, $\mathrm{Mg}$-doped $\mathrm{LiNbO}_{3}$ crystals were studied in order to suppress the strong absorption feature of small bound $\mathrm{Nb}_{\mathrm{Li}}^{4+}$ polarons (cf. Fig. 5). Based on these findings, Sasamoto et al. performed a systematic study on congruently melting and stoichiometric $\mathrm{Mg}$-doped $\mathrm{LiNbO}_{3}$, both for ordinary and extraordinary light polarization, and compared the results with appropriate nominally undoped samples. The small polaron formation time below 100 fs was verified; additionally, for the case of stoichiometric $\mathrm{Mg}$-doped $\mathrm{LiNbO}_{3}$, an increase of the number density of excited electrons of up to $N_{\mathrm{el}} \sim 1.2 \times 10^{24} \mathrm{~m}^{-3}$ was found being well below the typical number density of $\mathrm{Nb}_{\mathrm{Li}}^{5+}$ antisite defects in congruently melting $\mathrm{LiNbO}_{3}\left(\sim 30 \times 10^{24} \mathrm{~m}^{-3}\right)$. Furthermore, $N_{\mathrm{el}}$ is large in comparison with the small polaron number density of $N \approx 10^{23} \mathrm{~m}^{-3}$ (see Section IV B 1 ). Thus, it is concluded that only a fraction of optically excited carriers are trapped as small free polarons.

Fig. 12 shows the formation and relaxation dynamics of small bound $\mathrm{Nb}_{\mathrm{Li}}^{4+}$ polarons in the time regime from 0.24 picoseconds until 1 second after the excitation pulse by Beyer et al. according to Ref. 13. For this study, congruently grown, undoped $\mathrm{LiNbO}_{3}$ samples were used and studied in a pump-probe experiment described in Ref. 82 using 240 fs pulses at $388 \mathrm{~nm}$. The data show a pronounced peak around $t=0 \mathrm{ps}$, that is, attributed to two-photon absorption. Within this peak, the build-up of a transient absorption signal develops at a probing wavelength close to the maximum absorption feature of $\mathrm{Nb}_{\mathrm{Li}}^{4+}$; a first plateau is reached below $400 \mathrm{fs}$. The further decay follows a stretched exponential behavior over ten time decades. The spectra are inevitably assigned to $\mathrm{Nb}_{\mathrm{Li}}^{4+}$ due to the spectral, intensity, and time dependency.

\section{OPTICAL EXCITATION FROM POLARONIC CENTERS}

The optical excitation from polaronic centers has already been addressed in Section III. These considerations, however, were restricted to the simplified model of an optical transfer between next-neighboring polaronic sites. For the particular case of $\mathrm{LiNbO}_{3}$, this model must be refined as (i) the local structure of an initial polaronic site is surrounded by several final atomic sites that allow for an optical transfer into different directions and (ii) the optical excitation from a polaronic site may result in coupling to extended bandlike states, depending on the energy of the exciting photon. Both features result in a directed transport of carriers, resembling the bulk photovoltaic current density $\mathbf{j}_{\text {phv }}$. Together with the compensating, counter-directed incoherent current $\mathbf{j}_{\text {incoh }}$, characterized by the underlying hopping transport

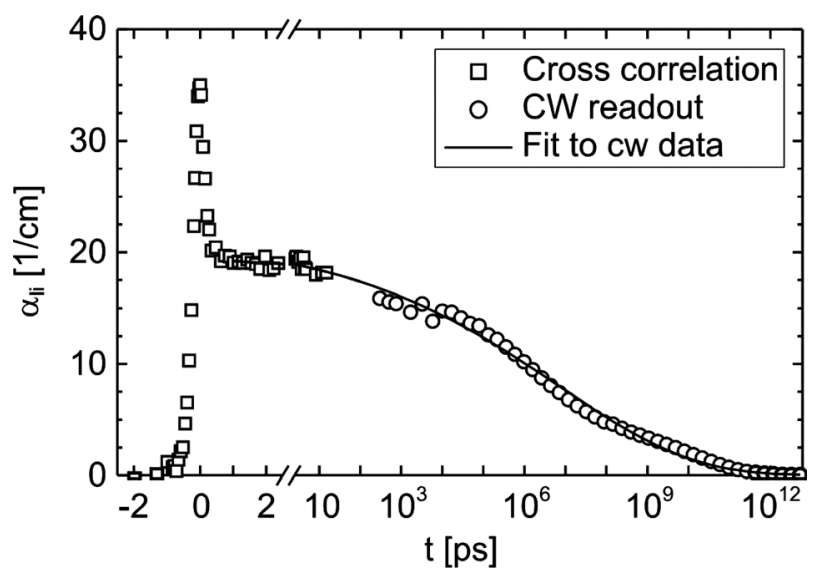

FIG. 12. Light-induced absorption $\alpha_{\mathrm{li}}$ versus the pump-probe delay $t$ detected at the wavelengths $\lambda_{\mathrm{r}}=776 \mathrm{~nm}$ up to $15 \mathrm{ps}$ and $\lambda_{\mathrm{r}}=785 \mathrm{~nm}$ starting $300 \mathrm{ps}$ after excitation with pump light of the wavelength $\lambda_{\mathrm{p}}=388 \mathrm{~nm}$ and the peak intensity $I_{\mathrm{p}}^{0} \approx 170 \mathrm{GW} / \mathrm{cm}^{2}$. The light polarization is extraordinary for pump and probe light. Around $t=0$, the time scale is linear; for the long-term evolution, the scale is logarithmic, starting from $t=3 \mathrm{ps}$. Reproduced with permission from Beyer et al., Appl. Phys. B 83, 085114 (2006). Copyright 2006 Springer Publishing. 
mechanism and, thus, a low mobility, the build-up of spacecharge fields with values up to $10^{5} \mathrm{~V} / \mathrm{cm}$ becomes possible and is reported for $\mathrm{LiNbO}_{3}{ }^{23}$ Such fields will be decisive for light-induced changes of the index of refraction that will be shown in Section VI on small-polaron based hologram recording.

The starting point of this section is Ref. 22, where the small-polaron based bulk photovoltaic effect is explained with Fe-doped $\mathrm{LiNbO}_{3}$ as an example and $\mathrm{Fe}^{2+}$ is considered as small polaronic center. Thus, and as $\mathrm{Fe}$ is incorporated on a Li site $\left(\mathrm{Fe}_{\mathrm{Li}}\right)$, the optical excitation from small $\mathrm{Nb}_{\mathrm{Li}}^{4+}$ or from $\mathrm{Nb}_{\mathrm{Li}}^{4+}: \mathrm{Nb}_{\mathrm{Nb}}^{4+}$ can be treated in the same manner as given by the following models.

\section{A. Directed optical transfer of small polarons from $\mathrm{Fe}_{\mathrm{Li}}$}

\section{Absorption features}

We first focus on the impact of local structure on the absorption spectrum $\alpha(\omega)$ that allows for understanding the complexity of the absorption shape as well as its dependence on the light polarization.

Fig. 13 indicates the relative atomic positions of $\mathrm{Fe}_{\mathrm{Li}}^{2+}$ and its eight $\mathrm{Nb}_{\mathrm{Nb}}^{5+}$ neighbors in the $y z$ glide mirror plane (for reasons of clarity, only two of the eight $\mathrm{Nb}^{5+}$ ions $(1,4)$ of the $x y$-plane are shown). We assume that $\mathrm{Fe}$ exactly replaces a Li site of the defect-free crystal ${ }^{98}$ and that the structural positions of $\mathrm{Li}$ and $\mathrm{Nb}$ remain unchanged with respect to the defect-free crystal. Each of the $\mathrm{Nb}_{\mathrm{Nb}}$ sites will be a possible

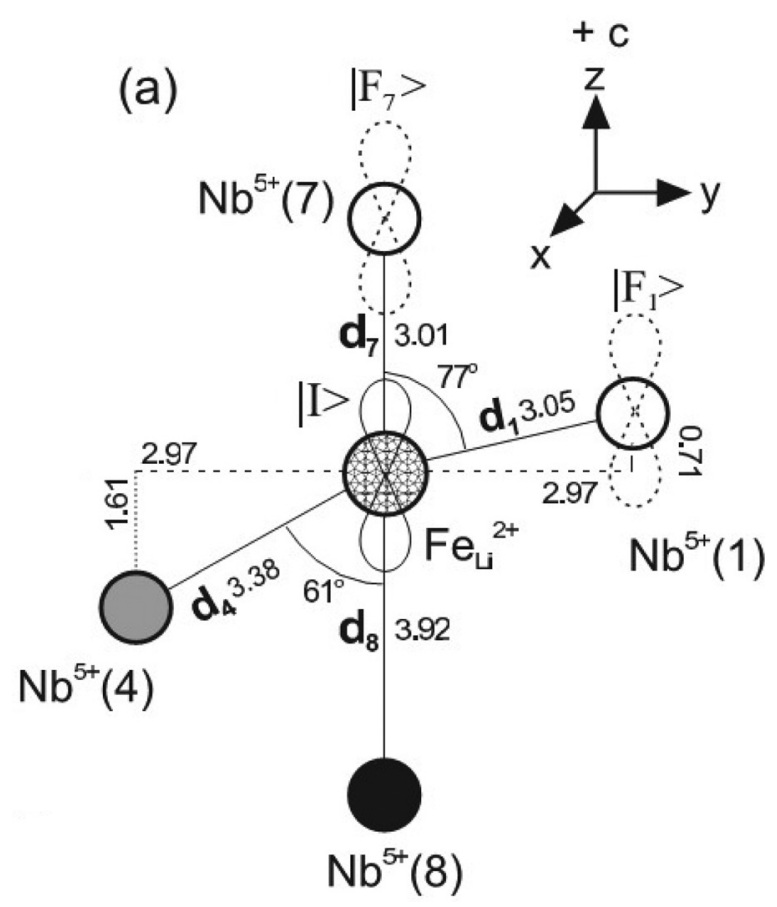

FIG. 13. Arrangement of $\mathrm{Fe}_{\mathrm{Li}}^{2+}$ and next-neighboring $\mathrm{Nb}_{\mathrm{Nb}}^{5+}$-ions in $\mathrm{LiNbO}_{3}$, assuming atomic positions unchanged with respect to the defect-free crystal (Ref. 98). The lengths of the interionic vectors $\mathbf{d}_{\mathbf{i}}$ are given in $A$. The picture shows a cut along a $y z$-glide mirror plane. For three sites, as examples, the axial structure (symmetry A1 in the $3 \mathrm{~m}$ group) of the involved orbitals of $\left(3 z^{2}-r^{2}\right)$ type are indicated. For $\mathrm{Fe}_{\mathrm{Li}}^{2+}\left(3 \mathrm{~d}^{6}\right)$, shown hatched, the orbital of the last electron is labeled $|\mathrm{I}\rangle$. For the $\mathrm{Nb}_{\mathrm{Nb}}^{4+}$ neighbors 1 and 7 , they are called $\left|\mathrm{F}_{1}\right\rangle$ and $\left|\mathrm{F}_{7}\right\rangle$. Reproduced with permission from Schirmer et al., Phys. Rev. B 83, 165106 (2011). Copyright 2011 APS Publishing. final location of an electron transferred optically from $\mathrm{Fe}_{\mathrm{Li}}^{2+}$. A single $\mathrm{Fe}-\mathrm{Nb}$ pair can thus serve as the elementary unit with which the basic facts of the absorption process (see Section III) can be elucidated. The orbital of the last electron in $\mathrm{Fe}_{\mathrm{Li}}^{2+}\left(3 \mathrm{~d}^{6}\right)$, also having axial symmetry $\mathrm{A}_{1},{ }^{99}$ will be symbolized by $|\mathrm{I}\rangle$. There is a finite probability that $|\mathrm{I}\rangle$ is leaking to each of its eight $\mathrm{Nb}_{\mathrm{Nb}}^{5+}$ neighbors described by effective transfer integrals $J_{i}(i=1, \ldots, 8)$. The absorption $\alpha_{i}(\omega)$ depends on the distances $d_{i}$ between $\mathrm{Fe}_{\mathrm{Li}}^{2+}$ and $\mathrm{Nb}_{\mathrm{Nb}}^{5+}$, and on $J_{i}$

$$
\alpha_{i}(\omega) \sim \frac{d_{i}^{2} J_{i}^{2}}{\omega} \cdot \exp \left[-\frac{\left(\hbar \omega-2 E_{p}-E_{C}\right)^{2}}{4 E_{p} \hbar \omega_{0}}\right],
$$

with the representative phonon energy $\hbar \omega_{0}$. Then, the observed optical absorption band of $\mathrm{Fe}_{\mathrm{Li}}^{2+}$ at about $2.6 \mathrm{eV}$ is described by $\sum_{i} \alpha_{i}$.

It is possible to understand the slight dependency of the resulting absorption spectrum for extraordinary and ordinary light, i.e., along and perpendicular to the polar $c$-axis. For this purpose, it is noteworthy that the magnitude of $\alpha_{i}$ is strongly dependent on the product $d_{i}^{2} J_{i}^{2}$, whereby $J_{i}(d)$ itself decreases in an exponential way as a function of the distance $d$. Thereby, transitions with small $d_{i}$ dominate the absorption spectrum, which are $d_{1,2,3}$ for ordinary and $d_{7}$ for extraordinary light. The difference in their length, however, is small, so that a weak polarization dependence of about $10 \%$ is to be expected and experimentally found. ${ }^{4}$

\section{Net currents}

In the next step, we analyze the appearance of a local current density along $c$ as a result of the optically induced, time-dependent charge transfer between $\mathrm{Fe}_{\mathrm{Li}}^{2+}$ and $\mathrm{Nb}_{\mathrm{Nb}}^{5+}$. Again, we consider the optical transitions along the different directions $d_{i}$, now restricted to their projections onto the $c$ direction. We assume the current density to be proportional to $\alpha_{i}$, neglect contributions originating from the displacement of ions, and get the following expression (summation over repeated indices is implied):

$$
\begin{aligned}
j_{z} \propto & \left\{\sum_{1,2,3,7}\left\langle I^{\prime}\left|r_{k}\right| F_{i}^{\prime}\right\rangle\left\langle F_{i}^{\prime}\left|r_{l}\right| I^{\prime}\right\rangle-\sum_{4,5,6,8}\left\langle I^{\prime}\left|r_{k}\right| F_{i}^{\prime}\right\rangle\left\langle F_{i}^{\prime}\left|r_{l}\right| I^{\prime}\right\rangle\right\} \\
& \times e_{k} e_{l}^{*}=j_{+c}-j_{-c} .
\end{aligned}
$$

Here, the matrix elements $\left\langle I^{\prime}\left|r_{k}\right| F_{i}^{\prime}\right\rangle$ and $\left\langle F_{i}^{\prime}\left|r_{l}\right| I^{\prime}\right\rangle$ represent the values expected for the respective transition dipole arms $r_{l}$ and $r_{k},{ }^{22}$ they are multiplied with the related components $e_{k}$ and $e_{l}^{*}$ of the polarization unit vectors of the electromagnetic waves. The sums of Eq. (21) add current densities parallel and anti-parallel to the $c$-direction. We further like to point to the analogy of Eq. (21) to the phenomenological expression of the bulk photovoltaic effect in $\mathrm{LiNbO}_{3}: j_{i}$ $=\beta_{i k l} e_{k} e_{l} * I$ with the third rank complex photovoltaic tensor $\beta_{i k l .}{ }^{100}$ According to the local defect structure depicted in Fig. 13, Eq. (21) simplifies assuming extraordinary light as, in this case, essential transitions along $d_{7}$ and $d_{8}$ are activated. Since the dipole changes along $d_{7}$ are expected to dominate due to its smaller distance (see above), a non-zero 
current density $j_{z}>0$ results. (Equivalent considerations are made in Ref. 22 to derive the current density $j_{y}$, but are omitted here, as only $j_{z}$ will be decisive for the further considerations of optical nonlinearities.) Equation (21) will be modified by additional terms, if current contributions originating from the displacement of the ions are considered. This, however, requires the precise knowledge of structural changes upon optically induced polaron transport. For the case of $\mathrm{Nb}_{\mathrm{Li}}^{4+} \rightarrow \mathrm{Nb}_{\mathrm{Li}}^{5+}$, Nahm and Park ${ }^{101}$ reported ionic displacements in the order of $0.04 \AA$ along the $c$-axis. A systematic study for $\mathrm{Fe}_{\mathrm{Li}}$ is on its way. ${ }^{102}$

As a consequence of the structural analysis, it becomes clear that also intrinsic defects, if located at $\mathrm{Li}$ sites in $\mathrm{LiNbO}_{3}$, will lead to comparable photovoltaic current densities. This explains that the optical absorption by a small bound polaron $\mathrm{Nb}_{\mathrm{Li}}^{4+}$ causes photovoltaic currents. ${ }^{61}$ The orbital topology of this defect is identical to that of $\mathrm{Fe}_{\mathrm{Li}}^{2+} \cdot{ }^{4,101}$ However, since the defect potential of $\mathrm{Nb}_{\mathrm{Li}}^{4+}$ is smaller $\left(E_{C}=0.53 \mathrm{eV}, E_{p}^{\prime}=0.58 \mathrm{eV}\right)$ than that of $\mathrm{Fe}_{\mathrm{Li}}^{2+}$ $\left(E_{C}=1.22 \mathrm{eV}, \quad E_{p}^{\prime}=0.70 \mathrm{eV}\right),{ }^{4}$ the transfer absorption cross section of $\mathrm{Nb}_{\mathrm{Li}}^{4+}$ is larger than that of $\mathrm{Fe}_{\mathrm{Li}}^{2+}$ (cf. Table I). This supports the increase of the photorefractivity in $\mathrm{LN}$, mainly determined by the bulk photovoltaic effect $^{28,29}$ if the $\mathrm{Nb}_{\mathrm{Li}}^{4+}$ charge state is populated. ${ }^{12}$ Moreover, the absorption cross section of the $\mathrm{Nb}_{\mathrm{Li}}^{4+}: \mathrm{Nb}_{\mathrm{Nb}}^{4+}$ bipolarons is about twice as large than that of $\mathrm{Nb}_{\mathrm{Li}}^{4+77}$ as the two electrons in the bipolaron ground state $^{4}$ cause a doubling of the oscillator strength (see Fig. 5), in line with the sum rule for oscillator strengths. Bipolarons in $\mathrm{LN}$ are therefore likely to lead to stronger photovoltaic currents, which is the starting point for our small-polaron based holographic studies ${ }^{35,36}$ presented in Section VI. In contrast, defects on regular $\mathrm{Nb}_{\mathrm{Nb}}$ sites are not expected to be active in the generation of photovoltaic current densities because the bonds to their twelve next-neighboring $\mathrm{Nb}_{\mathrm{Nb}}^{5+}$ ions have equal projections along the positive as well as the negative $c$ axis, i.e., $j_{z}=j_{+c}-j_{-c} \approx 0$.

Upon this first optical transition (usually on the sub-pstime scale, see Section IV B), it is very likely that the displaced electron at $\mathrm{Nb}_{\mathrm{Nb}}^{5+}$ will recombine with $\mathrm{Fe}_{\mathrm{Li}}^{3+}$ triggered by the generated, local electric field. Furthermore, as sketched in Fig. 14(a), there is no barrier for this decay in the potential landscape, i.e., recombination will occur via multiphonon emission without intermediate thermal excitation (see the Appendix of Ref. 22).

Considering only path 1 in Fig. 14(a), the current density described above would disappear within a few femtoseconds. However, electric fields up to $10^{5} \mathrm{~V} / \mathrm{cm}$ (Ref. 23) are well established to occur in congruently melting $\mathrm{LiNbO}_{3}$.

As schematically sketched in Fig. 14(a), we need to consider also a certain probability that the Franck-Condon excited electron, having energies resonant with the conduction band, tunnels away from the initial $\mathrm{Fe}_{\mathrm{Li}}$ site reached after optical transfer (path 2). Such a situation has been proven by experiments with very well-characterized model systems, $\mathrm{O}^{-}$hole small polarons in oxide materials. ${ }^{5}$ It should be noted that optical transfers exceeding the first $\mathrm{Nb}_{\mathrm{Nb}}$ coordination sphere around $\mathrm{Fe}_{\mathrm{Li}}$ may also contribute to the net current density $j_{z}$.
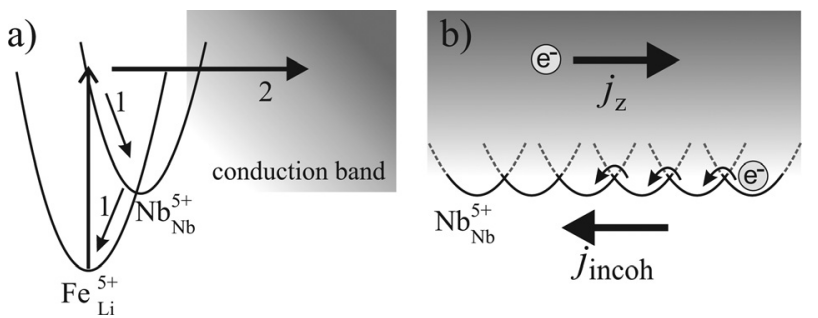

FIG. 14. (a) Scheme of the Fe-Nb potential landscape according to Ref. 22. Upon small polaron optical excitation from $\mathrm{Fe}_{\mathrm{Li}}^{2+}$ to $\mathrm{Nb}_{\mathrm{Nb}}^{5+}$, the electron is (1) moved forward to a potential centered around $\mathrm{Nb}_{\mathrm{Nb}}^{5+}$, followed by a relaxation of the Franck-Condon excited lattice to the new electronic state $\mathrm{Nb}_{\mathrm{Nb}}^{4+}$, accompanied by multi-phonon emission. Relaxation back to the potential landscape of $\mathrm{Fe}_{\mathrm{Li}}^{3+}$ occurs without intermediate thermal excitation. (2) The Franck-Condon excited electron, having energies resonant with the conduction band, tunnels away from the initial $\mathrm{Nb}_{\mathrm{Nb}}$ site. This transport is terminated by the formation of a small $\mathrm{Nb}_{\mathrm{Nb}}^{4+}$ polaron. (b) Excitations resonant with bandlike states cause the appearance of a high mobility transport (e.g., ballistic) expressed by current $j_{z}$. The mobility of the counter-directed, incoherent current $j_{\text {incoh }}$ is much smaller due to incoherent $3 \mathrm{D}$ hopping via $\mathrm{Nb}_{\mathrm{Nb}}$ (as shown) or $\mathrm{Nb}_{\mathrm{Li}}$ (and/or $\mathrm{Fe}_{\mathrm{Li}}$ ) (not shown).

As a result, the mixture between local and extended states is rather involved and strongly dependent on the energy of the exciting photon. For the case of a small bound polaron $\mathrm{Nb}_{\mathrm{Li}}^{5+}$, Nahm and Park ${ }^{101}$ have calculated the related excited energy levels lying in the conduction band continuum, all projected into a unit cell in $\mathbf{k}$ space, resulting from the combined influence of bandlike tunneling, the defect potential, and the polaron-type lattice distortions. Although rather complex, it becomes possible to understand the frequency dependence of the photovoltaic current density on the photon energy, represented by the spectral dependence of the Glass factor $\kappa(\omega):{ }^{31}$ At low photon energies, the influence of the local potential dominates; thus, the polaron absorption features are well described by polaron theory as presented in Section III. With increasing photon energy, the influence of band-like-states increases, resulting in a pronounced tail of $\kappa(\omega)$. This behavior is terminated as soon as the contribution of the band-to-band transition predominates. Consequently, the Glass factor shows a pronounced maximum, shifted to slightly higher energies $(3.1 \mathrm{eV})$ in comparison with the maximum of the small polaron absorption feature (see Fig. 5).

The transport processes described above are terminated by ionic displacements around the electron, forming a $\mathrm{Nb}_{\mathrm{Nb}}^{4+}$ free small polaron. The formation time of the $\mathrm{Nb}_{\mathrm{Nb}}^{4+}$ polarons from optically created non-thermalized electrons has been determined to be about $10^{-13} \mathrm{~s}$ at room temperature ${ }^{16,17}$ (see Section IV B). Their formation represents the initial step toward incoherent motion, since small polarons are characterized by hopping-type diffusive site changes (cf. Fig. 14(b)). For the $\mathrm{Nb}_{\mathrm{Nb}}^{4+}$ conduction polarons, the related activation energy is $0.27 \mathrm{eV} .{ }^{17}$ During their diffusion, they can also be trapped at defects having lower-lying levels, such as $\mathrm{Nb}_{\mathrm{Li}}^{5+}$ antisite defects present at $1 \%$ of all $\mathrm{Li}$ sites in congruently melting $\mathrm{LiNbO}_{3}$, or at one of the further impurities, such as $\mathrm{Fe}_{\mathrm{Li}}^{3+}$. These steps form a cascade of events ordered by characteristic time spans in the range from sub- $\mu$ s to seconds ${ }^{15,91}$ and are visualized experimentally by transient absorption spectroscopy shown in Section IV A 2. 
Incoherent hopping results by itself in a current density $\mathbf{j}_{\text {incoh }}$ (cf. Fig. 14(b)) but is characterized by a different, lower mobility in comparison to $j_{z}$. Because, however, $j_{z}$ is directed along $+c$, continuity requires that also the complete diffusive current $j_{\text {incoh }}$ is directed along $c$ (which is not a priori clear for a diffusive transport mechanism) and compensates for $j_{z}$ $\left(\left|j_{z}\right|=\left|j_{\text {incoh }}\right|\right)$. For an extended analytic treatment of these events, we thus refer to the related elaboration by Heyszenau. ${ }^{103}$ As a result, the open-circuit photovoltaic electric field is expressed by:

$$
E_{\mathrm{phv}}=\Lambda_{z}(\omega) / \mu \tau,
$$

with the mean transport length $\Lambda_{z}(\omega)$, the mobility of the incoherently transported electrons $\mu$, and their lifetime $\tau$. This field, which is as well dependent on the optical excitation from small bound polarons as on the mediation with band-like states, enables and explains efficient small-polaron based hologram recording with single ns-laser pulses, if cascaded excitation is taken into account, as it is demonstrated in Section VI.

\section{HOLOGRAM RECORDING BY MEANS OF SMALL POLARONS}

The optical features of small polarons in $\mathrm{LiNbO}_{3}$ presented, so far, offer two distinct nonlinearities for the recording and read-out of short-lived, thick volume holograms: either (i) by means of a population density modulation (of different types) of small polarons or (ii) via the small-polaron based photovoltaic effect, i.e., index modulations resulting from photovoltaic fields and the linear electro-optic effect. The features of respective holograms are expected to reflect the individual peculiarities of the underlying recording mechanisms, with the ultrafast nonlinear response over a broad spectral range being the most important one. Thus, small polaron based holograms particularly are of importance for the (developing) field of ultrafast, dynamical holography. Moreover, it is noteworthy that $\mathrm{LiNbO}_{3}$ thereby experiences a renaissance as fshologram recording material based on the variety of small polarons. Besides the issue of applications, holography is well suited for the study of optical nonlinearities and related transport mechanism. It will be shown that novel small polaron features are uncovered by means of holographic spectroscopy. ${ }^{104}$

The role of small polarons in holography has been addressed earlier by Jermann et al. within the two-center model. ${ }^{12}$ Here, the polaronic centers act as intermediate trap for the recording of holograms; in this case, the spatial modulation of the index of refraction is due to a charge redistribution between extrinsic defect centers and the electro-optic effect. Furthermore, it has been shown that small bound polarons may act as intermediate shallow traps in a multistep recording scheme for phase holograms featuring nondestructive read-out. ${ }^{41,42}$ Hesselink et al. were the first to deal with a spatial modulation density of $\mathrm{Nb}_{\mathrm{Li}}^{4+/ 5+}$ for hologram recording; ${ }^{41}$ although not using the language of small polarons at that time, their approach is already quite close to what we call small polaron based holography. The variety and complexity of hologram features reflecting the small polaron nature were not discovered until 2011. ${ }^{35,36}$ In what follows, the principle steps of small polaron based hologram recording based on the build-up of a spatial density modulations as well as on the small polaron-based photovoltaic effect are summarized with thermally reduced $\mathrm{LiNbO}_{3}$ as an example. These samples are particularly suited, as single (ultra-)short laser pulses in the blue-green spectral range can be applied to modulate the number densities of small bound polarons $\mathrm{Nb}_{\mathrm{Li}}^{4+}$, of hole polarons $N_{\mathrm{HP}}(x)$, and of bipolarons $N_{\mathrm{BP}}(x)$. In the first part of experimental studies presented below, the time resolution of the optical setup prevents the observation of small free $\mathrm{Nb}_{\mathrm{Nb}}^{4+}$ polarons; this, however, is acceptable as hologram recording is predominantly expected for modulated number densities of bound polarons as well as of the contribution of the polaron-based photovoltaic effect.

\section{A. Population density holograms}

As a prerequisite for hologram recording by means of small polaron density modulations, the action of the linear electro-optic effect needs to be excluded. This is realized by choosing the direction of the grating vector $\mathbf{K}$ perpendicular to the polar $c$-axis with light polarizations of pump and probe beams parallel to $c$ and $s$-polarization. Thereby, the recording without an electro-optic contribution, i.e., $r_{331}=0$, according to the point symmetry group $3 \mathrm{~m}$ of $\mathrm{LiNbO}_{3}$ was studied. ${ }^{105}$

\section{Hologram recording mechanism}

Taking into account small-polaron generation according to Section IV together with an exposure to an inhomogeneous, i.e., sinusoidal intensity pattern $I(x)=I_{\mathrm{p}}[1+\cos (|\mathbf{K}| x)]$, the appearance of spatially periodic density modulations of small bound electron and hole polarons as well as of bipolarons can be expected as sketched in Fig. $15 .{ }^{35}$ For the recording, we assume equal intensities of reference and signal beams $\left(I^{\mathrm{R}}=I^{\mathrm{S}}\right)$ yielding a modulation depth of $m=2 \sqrt{I_{\mathrm{R}} I_{\mathrm{S}}} /\left(I_{\mathrm{R}}+I_{\mathrm{S}}\right) \mathbf{e}_{\mathrm{p}}^{\mathrm{R}} \cdot \mathbf{e}_{\mathrm{p}}^{\mathrm{S}}=\mathbf{e}_{\mathrm{p}}^{\mathrm{R}} \cdot \mathbf{e}_{\mathrm{p}}^{\mathrm{S}}$. Here, $\mathbf{e}_{\mathrm{p}}^{\mathrm{R}}, \mathbf{e}_{\mathrm{p}}^{\mathrm{S}}$ denote the respective light polarizations. Furthermore, a symmetric angle of incidence $\Theta_{B}$ with respect to the sample's normal is assumed (unslanted grating ${ }^{106}$ ). Obviously, the resulting number densities $N_{\mathrm{GP}}(x)$ and $N_{\mathrm{HP}}(x)$ appear in phase to $I(x)$ while $N_{\mathrm{BP}}(x)$ is phase-shifted by $\pi$.

It is possible to probe these spatial density modulations via light diffraction by considering the related spatial modulations of absorption $\alpha(x)$ and index of refraction $n(x)$ that are mutually linked via Kramers-Kronig relation. ${ }^{20}$ Fig. 16 shows the overall spatial absorption modulation $\alpha(x)$ that can be expected from the individual contributions of bound $\alpha_{\mathrm{GP}}(x)$, hole $\alpha_{\mathrm{HP}}(x)$, and bipolarons $\alpha_{\mathrm{BP}}(x)$ at a probing wavelength $\lambda=785 \mathrm{~nm}$ and for extraordinary light polarization.

Here, we note that the pronounced band width of $\approx 1 \mathrm{eV}$ of small polaron absorption features enters the modeling, i.e., the tails of the absorption features of bipolarons and hole polarons at $785 \mathrm{~nm}$ are considered. Accordingly, a spatial modulation of the index of refraction $n_{1}(x)$ is generated, 


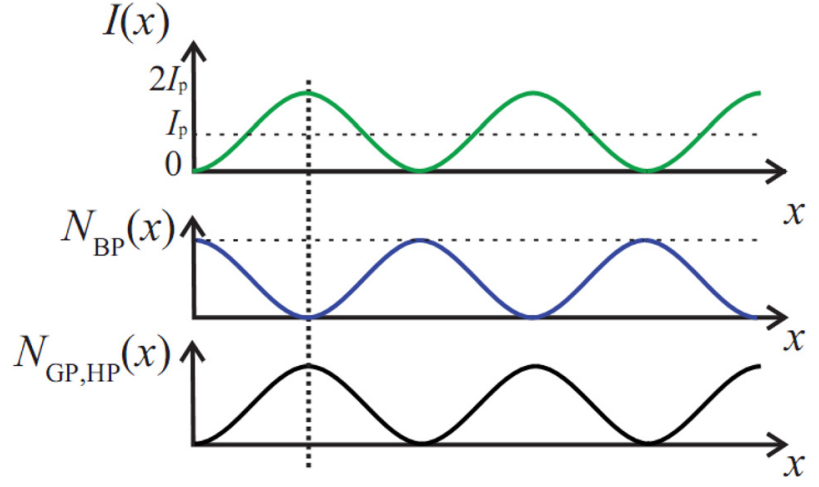

FIG. 15. Sinusoidal intensity pattern $I(x)$ applied for exposure in our experiments with average intensity $I_{\mathrm{p}}=I_{\mathrm{R}}+I_{\mathrm{S}}$ and modulation depth unity. Spatially periodic density modulations of small bound polarons $N_{\mathrm{GP}}(x)$, hole polarons $N_{\mathrm{HP}}(x)$, and bipolarons $N_{\mathrm{BP}}(x)$. Reproduced with permission from Imlau et al., Opt. Express 19, 15322 (2011). Copyright 2011 OSA Publishing.

as it results causally from Kramers-Kronig relation. It is in phase with $\alpha(x)$ and composed from individual index changes $n_{\mathrm{GP}}(x), n_{\mathrm{HP}}(x), n_{\mathrm{BP}}(x)$ related to the particular small polaron number densities.

For Bragg-incidence and the assumption of an unslanted, mixed (absorption and index) elementary holographic volume grating, with a mutual phase shift between light and absorption/index modulation of 0 or $\pi$, the diffraction efficiency can be expressed via Kogelnik's formula: ${ }^{106}$

$$
\begin{aligned}
\frac{I^{(1 \mathrm{st})}}{I_{0}}= & \exp \left(\frac{-2\left(\alpha+\alpha_{1}\right) \cdot d_{\mathrm{h}}}{\cos \Theta_{\mathrm{B}}}\right) \\
& \times\left[\sin ^{2}\left(\frac{\pi \cdot n_{1} \cdot d_{\mathrm{h}}}{\lambda \cos \Theta_{\mathrm{B}}}\right)+\sinh ^{2}\left(\frac{\alpha_{1} \cdot d_{\mathrm{h}}}{2 \cos \Theta_{\mathrm{B}}}\right)\right],
\end{aligned}
$$

with the effective thickness of the recorded hologram $d_{\mathrm{h}} \leq d$ and the Bragg angle in the medium $\Theta_{\mathrm{B}}$.

\section{Modeling}

Eq. (23) can be used to estimate the diffraction efficiency for the given experimental conditions of Fig. 18 (probe light at $\lambda=785 \mathrm{~nm}$, pump beam intensity $I_{\mathrm{p}}=380$

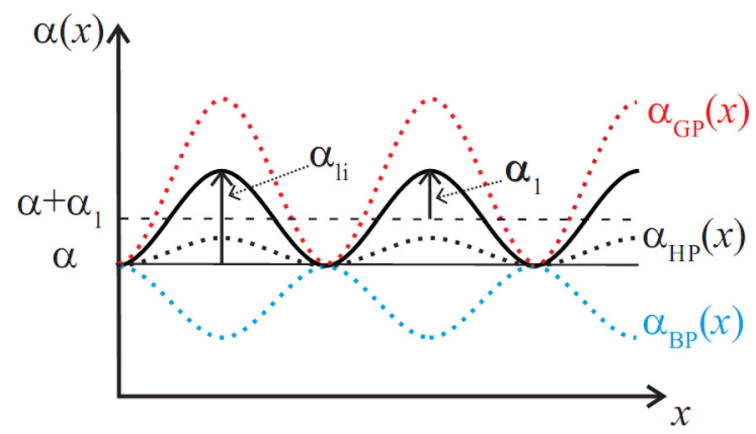

FIG. 16. Spatial modulation of the absorption coefficient $\alpha(x)$ with amplitude $\alpha_{1}$ and average value of $\alpha+\alpha_{1}$. The overall absorption change in the maximum of the fringe pattern $\alpha_{\mathrm{li}}=2 \alpha_{1}$ is composed by absorption changes of the individual polaron type: $\alpha_{\mathrm{li}, \mathrm{GP}}, \alpha_{\mathrm{li}, \mathrm{HP}}$ and $\alpha_{\mathrm{li}, \mathrm{BP}}$. All absorption contributions are related to $\lambda=785 \mathrm{~nm}$ and extraordinary light polarization. Reproduced with permission from Imlau et al., Opt. Express 19, 15322 (2011). Copyright 2011 OSA Publishing.
$\mathrm{GW} / \mathrm{m}^{2}$ and Bragg angle $\Theta_{\mathrm{B}}=11.5^{\circ}, \mathbf{K} \perp c$-axis, extraordinary light polarization and $s$-polarization) and the groundstate absorption $\alpha(785)=(270 \pm 10) \mathrm{m}^{-1}$ (cf. Table IV). For this purpose, we need to determine the maximum amplitudes of absorption and index modulations, $\alpha_{1}(785)$ and $n_{1}(785)$, as well as the hologram thickness $d_{\mathrm{h}}$.

The amplitude $\alpha_{1}(785)$ can be deduced from transient absorption spectra, as depicted in Fig. 11. Particularly, the initial value of the light-induced absorption $\alpha_{\mathrm{li}}(785)$ is to be identified with the value obtained within the intensity maximum of the fringe pattern $2 \cdot I_{\mathrm{p}}=760 \mathrm{GW} / \mathrm{m}^{2}$ and yields $\alpha_{1}(785)=\alpha_{\mathrm{li}}(785) / 2=(130 \pm 15) \mathrm{m}^{-1}$.

The amplitude $n_{1}(785)$ can be deduced from the application of the Kramers-Kronig relation to the spectral dependence $\alpha_{1}(\lambda)$. We model $\alpha_{1}(\lambda)$ as follows: The light-induced absorption is related to the changes of the number densities of light-induced bound polarons $N_{\mathrm{li}, \mathrm{GP}}$, bipolarons $N_{\mathrm{li}, \mathrm{BP}}$, and hole polarons $N_{\text {li,HP }}$ via:

$$
\begin{aligned}
\alpha_{\mathrm{li}}(\lambda) & =\alpha_{\mathrm{li}, \mathrm{GP}}(\lambda)+\alpha_{\mathrm{li}, \mathrm{HP}}(\lambda)+\alpha_{\mathrm{li}, \mathrm{BP}}(\lambda) \\
& =N_{\mathrm{li}, \mathrm{GP}} \cdot \sigma_{\mathrm{GP}}(\lambda)+N_{\mathrm{li}, \mathrm{HP}} \cdot \sigma_{\mathrm{HP}}(\lambda)+N_{\mathrm{li}, \mathrm{BP}} \cdot \sigma_{\mathrm{BP}}(\lambda),
\end{aligned}
$$

with

$$
N_{\mathrm{li}, \mathrm{GP}}=-\left(2 \cdot N_{\mathrm{li}, \mathrm{BP}}+N_{\mathrm{li}, \mathrm{HP}}\right) .
$$

Eq. (24) can be evaluated by considering the values of the light-induced absorption at the maximum of the individual polaron absorption bands, i.e., $\alpha_{\mathrm{li}}(785)$ and $\alpha_{\mathrm{li}}(488)$, that have been experimentally determined for the case of homogeneous exposure (Ref. 91, cf. Fig. 11). Further, the values of the individual absorption cross sections are required which can be deduced from Fig. 5. As a result, the lightinduced number densities listed in Table III have been obtained.

Now, the individual polaron line shapes of the optically induced absorption features can be determined using the band shapes of small polarons (cf. Section III). This allows to model the dispersion of the light-induced absorption $\alpha_{\mathrm{li}}(\lambda)$ over the visible and near-infrared spectral range as depicted in Fig. 17.

With the help of $\alpha_{\mathrm{li}}(\lambda)$, and neglecting uncertainties due to confined integral limits, the dispersion of the index change $n_{\mathrm{li}}(\lambda)$ is calculated via the Kramers-Kronig relation ${ }^{21}$

$$
n_{\mathrm{li}}(\omega)=\frac{2}{\pi} P \int_{0}^{\infty} \frac{\omega^{\prime} \Delta \kappa_{\mathrm{li}}\left(\omega^{\prime}\right)}{\omega^{\prime 2}-\omega^{2}} \mathrm{~d} \omega^{\prime},
$$

TABLE III. Estimated changes of the number densities of light-induced bound polarons $N_{\mathrm{li}, \mathrm{GP}}$, bipolarons $N_{\mathrm{li}, \mathrm{BP}}$, and hole polarons $N_{\mathrm{li}, \mathrm{HP}}$. Values are evaluated based on the experimental data shown in Fig. 11 and corresponding experimental conditions. Reproduced with permission from Imlau et al., Opt. Express 19, 15322 (2011). Copyright 2011 OSA Publishing.

\begin{tabular}{lc}
\hline Number densities & \\
\hline$N_{\mathrm{li}, \mathrm{GP}}$ & $(+60.0 \pm 15.0) \cdot 10^{22} \mathrm{~m}^{-3}$ \\
$N_{\mathrm{li}, \mathrm{BP}}$ & $(-26.0 \pm 10.0) \cdot 10^{22} \mathrm{~m}^{-3}$ \\
$N_{\mathrm{li}, \mathrm{HP}}$ & $(+7.6 \pm 3.0) \cdot 10^{22} \mathrm{~m}^{-3}$ \\
\hline \hline
\end{tabular}




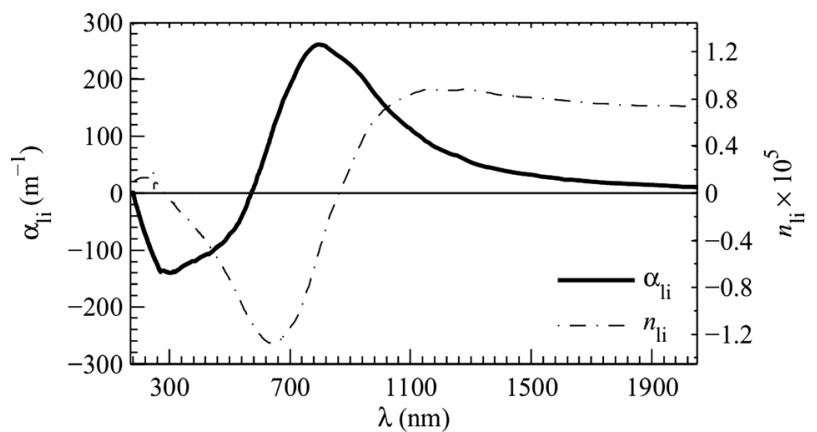

FIG. 17. Spectral dependence of $\alpha_{\mathrm{li}}(\lambda)$ determined from the analysis of the experimentally determined light-induced absorption at $785 \mathrm{~nm}$ and $488 \mathrm{~nm}$, the literature data on polaron absorption cross sections, and the previously published experimental band shapes of small polarons. ${ }^{5,57,66,107}$ The dispersion $n_{\mathrm{li}}(\lambda)$ is calculated from $\alpha_{\mathrm{li}}(\lambda)$ applying the Kramers-Kronig-relation, Eq. (26). For details, see text. Reproduced with permission from Imlau et al., Opt. Express 19, 15322 (2011). Copyright 2011 OSA Publishing.

where $P$ denotes the Cauchy principal value, $\Delta \kappa_{\mathrm{li}}$ $=\alpha_{\mathrm{li}}(\omega) \cdot c /(2 \cdot \omega), \omega=2 \cdot \pi \cdot c / \lambda$, and $c$ is the vacuum speed of light. The resulting spectrum is plotted as dashed line in Fig. 17. From this dependence, we find an amplitude of the light-induced refractive index modulation at our probe wavelength of $n_{1}(785)=n_{\mathrm{li}}(785) / 2=(-3.0 \pm 1.0) \cdot 10^{-6}$.

Finally, Eq. (23) requires an estimate for the hologram thickness $d_{\mathrm{h}} \leq d$. This value is taken from the analysis of the rocking curve in the configuration $\mathbf{K} \| c$-axis (cf. Fig. 22) to $d_{\mathrm{h}}=(0.62 \pm 0.07) \mathrm{mm}$. As it is smaller than the crystal thickness due to absorption losses of the diffracted beam within the crystal volume, it becomes necessary to modify the estimate of the diffraction efficiency via Eq. (23) by an additional term $\exp \left\{-2\left(\alpha+\alpha_{1}\right)\left(d-d_{h}\right) / \cos \theta_{B}\right\}$. All values necessary for the calculation and the determined value for $I^{(1 s t)} / I_{0}$ are summarized in Table IV.

According to our model, the bipolaron density is reduced via optical gating processes in the bright regions of the fringe pattern and yields an increase of the number density of small bound polarons by $N_{\mathrm{li}, \mathrm{GP}}$ with

$$
N_{\mathrm{li}, \mathrm{GP}}=2 N_{\mathrm{li}, \mathrm{BP}}
$$

Here, $N_{\text {li,BP }}$ denotes the number density of optically gated bipolarons. Because of the limited number density of bipolarons $N_{\mathrm{BP}}$ in the groundstate, a saturation behavior according to

TABLE IV. Modelling output for $I^{(1 s t)} / I_{0}$ via Eq. (23) and a small-polaron based hologram with $\mathbf{K} \perp c$-axis, light polarization parallel $c$-axis, and $s$ polarization. The index of refraction $n(785)$ is taken from Ref. 108, see text for details. Reproduced with permission from Imlau et al., Opt. Express 19, 15322 (2011). Copyright 2011 OSA Publishing.

\begin{tabular}{lc}
\hline Parameters entering Eq. (23) & \\
\hline$\alpha(785)$ & $(270 \pm 10) \mathrm{m}^{-1}$ \\
$\alpha_{\mathrm{li}}(785)$ & $(260 \pm 15) \mathrm{m}^{-1}$ \\
$\alpha_{1}(785)=\alpha_{\mathrm{li}}(785) / 2$ & $(130 \pm 15) \mathrm{m}^{-1}$ \\
$n(785)$ & $(2.1776 \pm 0.0005)$ \\
$n_{1}(785)=n_{\mathrm{li}}(785) / 2$ & $(-3.0 \pm 1.0) \cdot 10^{-6}$ \\
$d_{\mathrm{h}}$ & $(0.62 \pm 0.07) \mathrm{mm}$ \\
$I^{(11 s t)} / I_{0}$ & $(6.0 \pm 2.0) \cdot 10^{-4}$ \\
\hline \hline
\end{tabular}

$$
N_{\mathrm{li}, \mathrm{BP}}=N_{\mathrm{BP}}\left[1-\exp \left(\frac{-I_{\mathrm{p}}}{I_{\mathrm{c}}}\right)\right]
$$

is to be expected and has been experimentally verified. ${ }^{91}$ Here, $I_{\mathrm{p}, \mathrm{c}}$ denote pump and characteristic intensities, see also Fig. 9. Because of the relation given by Eq. (27), the intensity dependence of small bound polarons shows saturation with the same characteristic intensity $I_{\mathrm{c}}$, but an amplitude by a factor of two larger compared with Eq. (28). Furthermore, the absorption amplitude and the increase of polaron number density are directly linked via the absorption cross section. Hence, saturation as a function of pump beam intensity also appears for the amplitudes $\alpha_{1, \mathrm{BP}, \mathrm{GP}, \mathrm{HP}}$ and, taking into

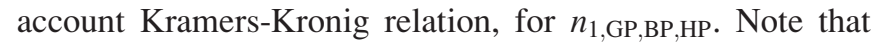
small polaron formation via two-photon absorption is neglected; thus, for large intensities, the diffraction efficiency inevitably saturates. For small amplitudes $\alpha_{1}, n_{1}$ and $\alpha_{1} \ll \alpha_{0}$, its intensity dependence can be approximated by

$$
\eta\left(I_{\mathrm{p}}\right) \approx\left(c_{1} n_{1}\left(I_{\mathrm{p}}\right)\right)^{2}+\left(c_{2} \alpha_{1}\left(I_{\mathrm{p}}\right)\right)^{2}=\eta^{\text {sat. }}\left[1-\exp \left(\frac{I_{\mathrm{p}}}{I_{\mathrm{c}}}\right)\right]^{2},
$$

determined solely by two fitting parameters: the saturation value $\eta^{\text {sat. }}$ and the characteristic intensity $I_{c}$.

\section{Results and analysis}

Fig. 18 highlights the temporal dynamics of the first order diffracted probe beam obtained after hologram recording within a classical two-beam interferometer at a probing wavelength of $785 \mathrm{~nm}$. For efficient generation of small polaron number densities, a single pulse of a frequency-doubled YAG:Nd-laser (Innolas Spitlight 600, $\left|\mathbf{k}_{\mathrm{p}}\right|=2 \pi / \lambda_{\mathrm{p}}, \lambda_{\mathrm{p}}$ $=532 \mathrm{~nm}$, maximum applied intensity $I_{\mathrm{p}}^{\max }=760 \mathrm{GW} / \mathrm{m}^{2}$,

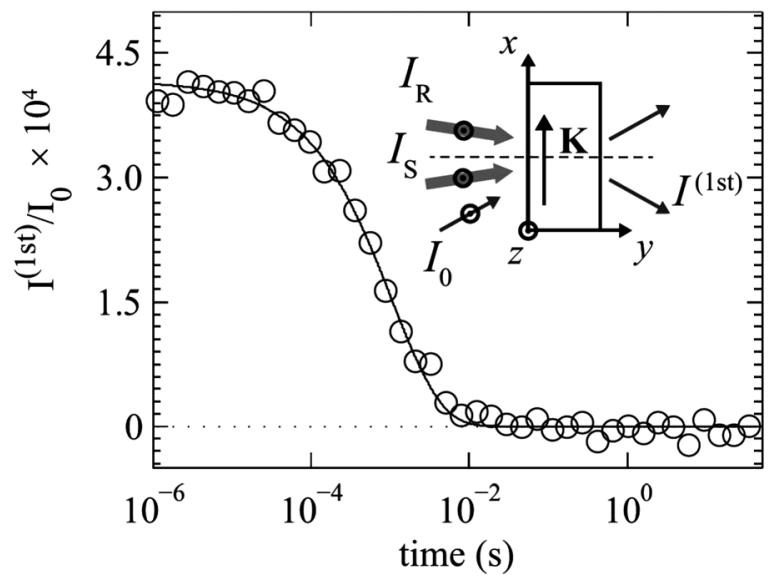

FIG. 18. Temporal dynamics of the intensity of the first order diffracted beam $I^{(1 \mathrm{st})}$ for $\mathbf{K} \perp c$-axis, $\mathbf{e}_{\mathrm{p}} \| c$-axis, $s$-polarization. Recording conditions: $\lambda_{\mathrm{p}}=532 \mathrm{~nm}, \Theta_{\mathrm{B}}=11.5^{\circ}$, spatial frequency $\Lambda=1.4 \mu \mathrm{m}, I_{\mathrm{p}}=I_{\mathrm{R}}+I_{\mathrm{S}}=$ $380 \mathrm{GW} / \mathrm{m}^{2}$, and $230 \mathrm{GW} / \mathrm{m}^{2}$, respectively. Bragg-matched probing conditions: $\lambda=785 \mathrm{~nm}$, e $\| c$-axis with $s$-polarization. The data are normalized to the intensity of the incoming probe beam $I_{0}$, and a logarithmic time scale is applied. The straight lines correspond to a fit of a stretched-exponential function Eq. (18) to the data set. The insets sketch the respective recording and probing configurations. Reproduced with permission from Imlau et al., Opt. Express 19, 15322 (2011). Copyright 2011 OSA Publishing. 
$10 \mathrm{~Hz}$ repetition rate, and average pulse duration $\tau_{\mathrm{FWHM}} \approx$ $8 \mathrm{~ns}$, Gaussian spatial beam profiles $2 \omega_{0} \approx 4 \mathrm{~mm}$ ) was used. The pulse was split by a 50:50-beam splitter; i.e., equal intensities were adjusted along the two optical paths $\left(I^{\mathrm{R}}=I^{\mathrm{S}}\right)$ yielding a modulation depth of $m=2 \sqrt{I_{\mathrm{R}} I_{\mathrm{S}}} /$ $\left(I_{\mathrm{R}}+I_{\mathrm{S}}\right) \mathbf{e}_{\mathrm{p}}^{\mathrm{R}} \cdot \mathbf{e}_{\mathrm{p}}^{\mathrm{S}}=\mathbf{e}_{\mathrm{p}}^{\mathrm{R}} \cdot \mathbf{e}_{\mathrm{p}}^{\mathrm{S}}$. Here, $\mathbf{e}_{\mathrm{p}}^{\mathrm{R}}, \mathbf{e}_{\mathrm{p}}^{\mathrm{S}}$ denote the respective light polarizations. A symmetric angle of incidence $\Theta_{B}$ was chosen with respect to the sample's normal. A fiber-coupled GaAlAs-diode laser (Coherent Cube, $|\mathbf{k}|=2 \pi / \lambda, \lambda=785 \mathrm{~nm}$ ) served for Bragg-matched as well as off-Bragg continuous wave (cw) probing. Its intensity was limited to $I_{0}=10 \mathrm{~kW} / \mathrm{m}^{2}$, thus not affecting the polaron-density dynamics.

In Fig. 18 , the intensity $I^{(1 \mathrm{st})}(t)$ is normalized to the intensity of the incoming probe beam $I_{0}$ and is to be identified with the diffraction efficiency $\eta$. It is plotted on a logarithmic time scale and characteristically shows a non-exponential decay to zero. Fitting a stretched exponential function according to the empirical dielectric decay function by Kohlrausch, Williams, and Watts (KWW), ${ }^{86}$

$$
\frac{I^{(1 \mathrm{st})}(t)}{I_{0}}=\frac{I^{(1 \mathrm{st})}(t=0)}{I_{0}} \exp \left[-(t / \tau)^{\beta}\right],
$$

to the experimental data (solid line in Fig. 18) yields the starting amplitude $I^{(1 \mathrm{st})}(t=0) / I_{0}$, the decay time $\tau$, and the stretching coefficient $\beta$ given in Table $\mathrm{V}$.

By comparison, a striking coincidence between the estimate $\eta \approx 6 \cdot 10^{-4}$ with the experimentally determined value of $\eta=(4.2 \pm 1.0) \cdot 10^{-4}$ is obvious. Furthermore, from temperature dependent measurements $\tau(T)$ in the temperature regime $300-410 \mathrm{~K}$, and by using an Arrhenius plot, an activation energy $\mathrm{E}_{\mathrm{a}}$ (see Table $\mathrm{V}$ ) of $\bar{E}_{\mathrm{a}}=(0.57 \pm 0.07) \mathrm{eV}$ has been determined. This activation energy also is in good agreement with the model approach as it reflects the barrier height of thermally activated hopping of small bound polarons.

In the next step, the dependence of the starting amplitude on the intensity of the recording beams is verified, see Fig. 19. ${ }^{36}$ The diffraction efficiency saturates at a value of about $1.8 \cdot 10^{-4}$ and pump beam intensities larger than $200 \mathrm{GW} / \mathrm{m}^{2}$ and can be described well by equation (29) (solid line in figure).

Appropriate experimental studies have been performed with probing light in the blue-green spectral range at $488 \mathrm{~nm}$ (Ref. 36) while keeping all other conditions. In this case, the contributions of the optical features of particular polarons differ according to their absorption fingerprints in Fig. 5. As

TABLE V. Parameters obtained by fit of Eq. (30) to the data of Fig. 18. The activation energies $\mathrm{E}_{\mathrm{a}}$ were determined via an Arrhenius plot from temperature dependent spectra. Reproduced with permission from Imlau et al., Opt. Express 19, 15322 (2011). Copyright 2011 OSA Publishing.

\begin{tabular}{lc}
\hline \hline & $\mathbf{K} \perp c$-axis \\
\hline$I^{(1 \mathrm{st})}(t=1 \mu s) / I_{0}$ & $(4.2 \pm 1.0) \cdot 10^{-4}$ \\
$\tau$ & $(1.1 \pm 0.3) \mathrm{ms}$ \\
$\beta$ & $(0.69 \pm 0.20)$ \\
$E_{\mathrm{a}}$ & $(0.55 \pm 0.07) \mathrm{eV}$ \\
\hline \hline
\end{tabular}

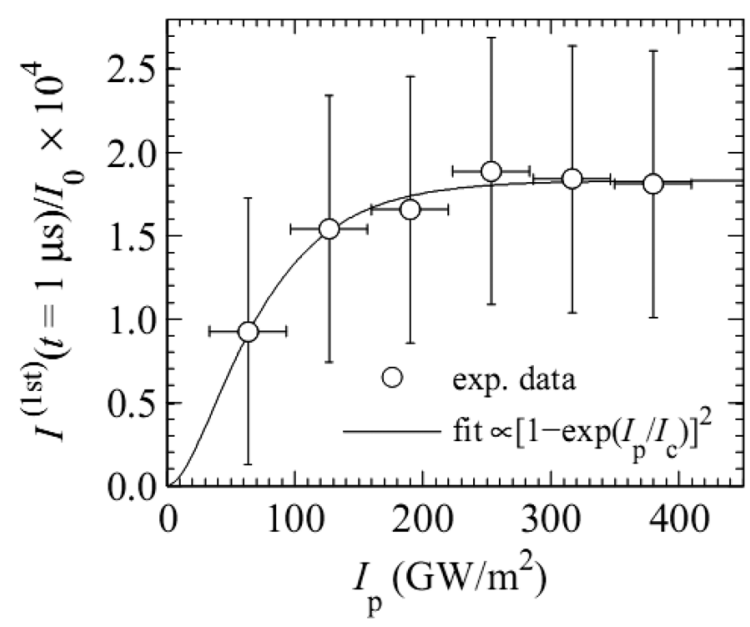

FIG. 19. Normalized intensity of the first order diffracted beam $I^{(1 \mathrm{st})} / I_{0}$ at $t=1 \mu \mathrm{s}$ as a function of pump intensity $I_{\mathrm{p}}$ for $\mathbf{K} \perp c$-axis (s-polarization). The solid line corresponds to a fit of a saturation function Eq. (29) with $\eta^{\text {sat. }}=(1.8 \pm 0.8) \cdot 10^{-4}$ and $I_{\mathrm{c}}=(52 \pm 10) \mathrm{GW} / \mathrm{m}^{2}$. For details, see text. Reproduced with permission from Bruening et al., Opt. Express 20, 13326 (2012). Copyright 2012 OSA Publishing.

the dispersive features of the latter are known, it is possible to model the dispersive behavior of the diffraction efficiency over the entire blue-green spectral range. Fig. 20 shows the results of modeling together with the experimentally determined values. Coincidence is found within the experimental error. Moreover, as a specific feature of the underlying small polaron based hologram recording mechanism, abnormal dispersion behavior of $\eta$ is found. This is mainly due to the pronounced small polaron absorption bands in the visible spectral range. As a result, the mutual contribution of lightinduced index and absorption changes vary from 400 to

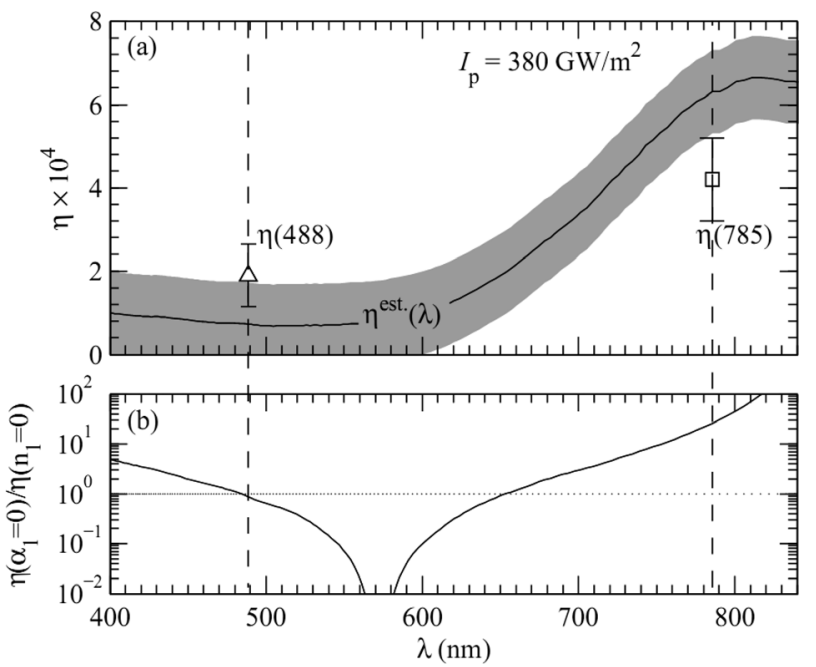

FIG. 20. (a) Dispersion of the diffraction efficiency $\eta_{\text {est. }}$ (solid line) that has been estimated according to Eq. (3) and the parameters published in Ref. 35. The grey area denotes the error for $\eta_{\text {est. }}$. The experimentally determined efficiencies at a probing wavelength of $488 \mathrm{~nm}$ and $785 \mathrm{~nm}$ have been added for comparison. (b) Dispersion of the ratio of the diffraction efficiency for a pure absorption grating and a pure index grating. A predominant contribution of the absorption grating is found at $785 \mathrm{~nm}$ while amplitude and index grating likewise contribute to the overall efficiency at $488 \mathrm{~nm}$. Reproduced with permission from Bruening et al., Opt. Express 20, 13326 (2012). Copyright 2012 OSA Publishing. 
$900 \mathrm{~nm}$; within the spectral window of 480-655 nm, the presence of a pure index grating can be assumed. This is of importance particularly for applications, as efficient holograms can be recorded in this spectral range or the impact of optical lensing by small polarons becomes apparent, here (see Section VII).

\section{B. Holograms by means of the electro-optic effect}

The key difference for hologram recording with $\mathbf{K} \| c$ axis is that the action of the linear electro-optic coefficient with non-vanishing tensor elements $r_{113} \neq 0$ and $r_{333} \neq 0$ needs to be considered. In this case, index modulations may arise in presence of an electrical field $E$ via

$$
n_{1}(\lambda)=-\frac{1}{2} n_{\mathrm{eff}}^{3}(\lambda) r_{\mathrm{eff}}(\lambda) E,
$$

which is valid for index changes in crystals of the point symmetry $3 \mathrm{~m}$. Here, $n_{\text {eff }}(\lambda)$ and $r_{\text {eff }}(\lambda)$ denote the effective index of refraction and the effective electro-optic tensor element at wavelength $\lambda$.

How can the appearance of an internal electric field be explained using the small-polaron approach? This question has been addressed in its detail in Section V based on microscopic considerations of optically induced charge transfer from a polaronic to its next-neighboring site. The structural environment within the first coordination sphere of the $\mathrm{Nb}_{\mathrm{Li}}^{4+}$ site is responsible for the appearance of a net current (see Section VA 2). In addition, there is a probability that the Franck-Condon excited electrons may tunnel away from its initial polaronic site, if its energy becomes resonant with the conduction band. In such a case, the unidirectional carrier transport length $\Lambda_{\text {ballistic }}^{\text {uni }}$ exceeds by far the first coordination sphere. By means of Monte-Carlo Simulations to describe the dynamics of the counter-directed incoherent hopping transport, Carnicero et al. succeeded in modeling of the experimental data with the assumption of $\Lambda_{\text {ballistic }}^{\text {uni }} \approx 1-3 \mathrm{~nm}$ for Fe-doped $\mathrm{LiNbO}_{3}$, being strongly dependent on the pump intensity and stoichiometry. ${ }^{18}$ This transport length should not be mixed up with the mean transport length $\Lambda_{z}$ determined by Glass et al. $(\approx 1 \AA)$ (Ref. 23 ) and applied for the estimate of the photovoltaic field strength. The latter takes charge displacements in both $+c$ - and $-c$-directions into account, thus expressing the net charge displacement and determining the net current density $j_{z}$. In contrast, for holography, the uni-directional carrier displacement $\Lambda_{\text {ballistic }}^{\text {uni }}$ is decisive: Considering the recording of a transmission phase hologram in the visible spectral range, we are dealing with grating constants of the fringe pattern in the regime from $\Lambda_{\text {grating }} \approx 500-5000 \mathrm{~nm}$. For the build-up of an electric field that modulates the index of refraction via the Pockel's effect, it is necessary to redistribute carriers from bright to dark regions, i.e., over distances of up to $\Lambda_{\text {grating }} / 2$. Within a single event of optical excitation and $\Lambda_{\text {ballistic }}^{\text {uni }}$, that is, in the order of a few nm as stated above, it is thus not possible to record an (efficient) hologram. For this purpose, cascaded excitation from polaronic centers needs to be considered, i.e., the repeated optical excitation and transfer of a carrier from one polaronic site to the other during the light pulse. In such a way, by means of hundred-fold re-excitation, carriers may get over distances of several hundreds of nanometers. Another origin for long-range transport may be impactionization as proposed by Garcia-Lechuga et al. ${ }^{109}$

It is possible to roughly estimate the pulse duration necessary for the recording of a phase hologram with maximum efficiency, i.e., described by a photovoltaic field according to Eq. (22). The total duration of a laser pulse $\tau_{\text {pulse }}$ is determined by the single transfer time between two polaronic sites under illumination $\tau_{\text {transfer }}$ in product with the number of reexcitation processes $N_{\text {casc }}: \tau_{\text {pulse }}=\tau_{\text {transfer }} \times N_{\text {casc }}$. The single transfer time $\tau_{\text {transfer }}$ can be identified with the polaron formation time as experimentally determined using fs-spectroscopy $^{13,17,81}$ and summarized in the previous Section IV B. Although not determined for the optical excitation from polaronic sites itself, so far, we do not expect different orders of magnitudes for the formation times and, here, assume $\tau_{\text {transfer }} \approx 100 \mathrm{fs}$. With $N_{\text {casc }} \approx 100$, we get $\tau_{\text {pulse }}=10$ ps. It is thus likely that efficient holograms based on smallpolarons can be recorded using ps- and ns-laser pulses, while recording with pulse durations from $0.1-10$ ps will be less efficient, although possible. The latter has been demonstrated by Reckenthaeler et al. using 200 fs laser pulses and it is shown that the gratings recorded with such small pulse durations are mainly due to free carriers. ${ }^{110}$ In Ref. 111 , Gamaly et al. recorded gratings in stoichiometric $\mathrm{LiNbO}_{3}$ with a characteristic lifetime of $0.1-1 \mathrm{~s}$ that points to a contribution of small polarons.

We like to note that the aspect of temporal recording dynamics marks the most striking difference to the classical photorefractive effect. ${ }^{34}$ Conductivity and dielectric permittivity determine the photorefractive recording time. The crossing point then is the carrier mobility of small polarons. Keeping this in mind, the principle mechanism for hologram recording using the polaron-based photovoltaic effect is

$$
I(z) \rightarrow j_{z}(z) \rightarrow \rho_{\text {polaron }}(z) \rightarrow E_{\mathrm{phv}}(z) \rightarrow n(z),
$$

with the polaron density $\rho_{\text {polaron }}(z)$.

\section{Results}

A striking feature of hologram recording by means of the small-polaron based photovoltaic effect is the possibility to record efficient phase gratings within a single picosecond or nanosecond laser pulse in nominally undoped $\mathrm{LiNbO}_{3}$. Experimental studies ${ }^{35,36}$ verify further features, as it is demonstrated in the following for ns-laser pulse experiments.

In order to get a dominant contribution of the smallpolaron based photovoltaic effect, thermally reduced samples of $\mathrm{LiNbO}_{3}$ are applied for the studies. Then, a pronounced number density of small bound $\mathrm{Nb}_{\mathrm{Li}}^{4+}: \mathrm{Nb}_{\mathrm{Nb}}^{4+}$ bipolarons are present that act as a source for carrier excitation. The photon energy of the laser pulse is chosen such that both optically induced transfer to the next-neighboring polaron site (that is a regular $\mathrm{Nb}_{\mathrm{Nb}}^{5+}$ site) and tunneling via an energetic state resonant with the conduction band become possible. According to the dispersion behavior of the Glass constant, and the bipolaron absorption feature, this is verified by $E_{\text {pump }}=2.5 \mathrm{eV}$ 
$(532 \mathrm{~nm})$, for which a frequency-doubled YAG:Nd-laser ( $\tau_{\text {pulse }} \approx 8 \mathrm{~ns}$ ) is used. It should be added that the net current $j_{z}$ may be larger in comparison with $\mathrm{Fe}_{\mathrm{Li}}$ or $\mathrm{Nb}_{\mathrm{Li}}$ due to the capture of two electrons that induce a stronger lattice distortion.

Fig. 21 exemplarily shows the result of a dynamic measurement of the diffraction efficiency in thermally reduced, nominally undoped $\mathrm{LiNbO}_{3}$ upon recording an elementary grating in transmission geometry, for $\mathbf{K} \| c$-axis, and with a single ns-laser pulse at $532 \mathrm{~nm}$. The probing light was chosen to a wavelength of $785 \mathrm{~nm}$, i.e., at the maximum of the small bound polaron absorption feature (see Fig. 5).

A remarkable diffraction efficiency of up to $\eta=(0.23$ $\pm 0.05)(0 \leq \eta \leq 1)$ was obtained; it decayed to zero in a non-exponential way on the ms-time scale. These values are not in accordance with $\eta=(2.5 \pm 1.0) \cdot 10^{-4}$ and $n_{1}(785)=$ $(-1.8 \pm 1.0) \cdot 10^{-6}$ that can be estimated from the lightinduced absorption features and Kramers-Kronig relation (Eq. (26)) analogous to the considerations of Section VI A 2. Hence, an effect that contributes to a light-induced index change in excess of Kramers-Kronig relation must be considered.

Because of the pronounced efficiency and the complete self-erasure of the lifetime-limited recorded grating, it became possible to record a rocking curve, i.e., the diffraction efficiency as a function of angular detuning at a given time delay of $1 \mu \mathrm{s}$ after the ns-laser pulse. The detuning results in dephasing of waves that constitute the diffracted beam, such that the integral amplitude decreases. ${ }^{112}$ For this purpose, the recording was repeated using the same experimental configuration except the angle of the probing beam that was detuned by $\delta \theta_{\mathrm{B}}$ in a range of $\pm 0.5^{0}$ with respect to the Bragg angle $\theta_{\mathrm{B}}$. Then, the values of $\eta$ at a delay time of $1 \mu \mathrm{s}$ with respect to the pulse were deduced from each singular measurement and plotted as a function of $\delta \theta_{\mathrm{B}}$. The result

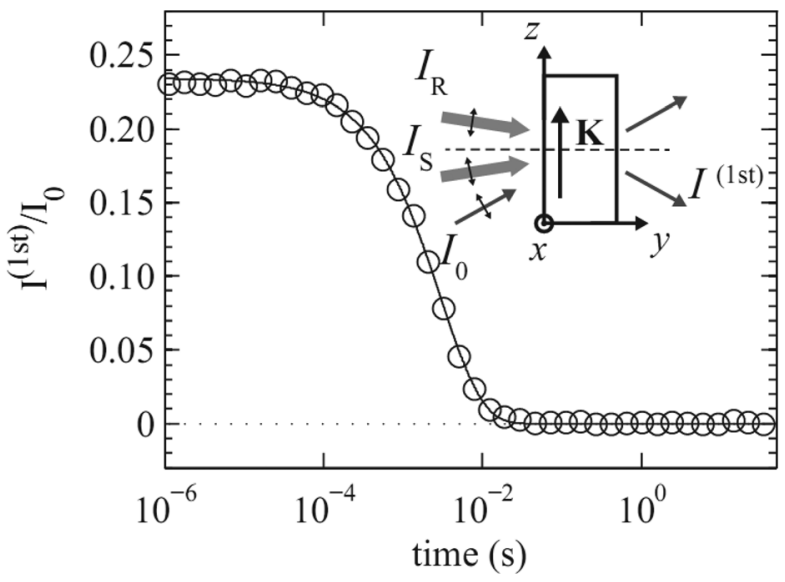

FIG. 21. Temporal dynamics of the intensity of the first order diffracted beam $I^{(1 \text { st })}$ for $\mathbf{K} \| c$-axis, $\mathbf{e}_{\mathrm{p}} \| c$-axis, and $p$-polarization. Recording conditions: $\lambda_{\mathrm{p}}=532 \mathrm{~nm}, \Theta_{\mathrm{B}}=11.5^{\circ}$, spatial frequency $\Lambda=1.4 \mu \mathrm{m}, I_{\mathrm{p}}=I_{\mathrm{R}}+I_{\mathrm{S}}$ $=380 \mathrm{GW} / \mathrm{m}^{2}$, and $230 \mathrm{GW} / \mathrm{m}^{2}$, respectively. Bragg-matched probing conditions: $\lambda=785 \mathrm{~nm}, \mathbf{e} \| c$-axis with $p$-polarization. The data are normalized to the intensity of the incoming probe beam $I_{0}$, and a logarithmic time scale is applied. The straight lines correspond to a fit of a stretched-exponential function Eq. (18) to the data set. The insets sketch the respective recording and probing configurations. Reproduced with permission from Imlau et al., Opt. Express 19, 15322 (2011). Copyright 2011 OSA Publishing. is depicted in Fig. 22. A rather pronounced dependence $\eta\left(\delta \theta_{\mathrm{B}}\right)$ is obvious with a characteristic full width at half maximum of $\approx 0.5^{\circ}$. Due to the pronounced value of $\eta$ exceeding the maximum value of a lossy grating, the rocking curve is fitted by coupled-wave theory for a pure phase grating 104,106

$$
\frac{I^{(1 \mathrm{st})}(\delta \Theta)}{I_{0}}=\exp \left(\frac{-2\left(\alpha+\alpha_{1}\right) d_{\mathrm{h}}}{\cos \Theta_{\mathrm{B}}}\right) \sin ^{2}\left(\frac{\sqrt{\nu^{2}+\zeta^{2}}}{1+\zeta^{2} / \nu^{2}}\right)
$$

with

$$
\nu=\pi n_{1} d_{\mathrm{h}} \cos \left(2 \Theta_{B}\right) /\left(\lambda \cos \Theta_{\mathrm{B}}\right)
$$

and

$$
\zeta=2 \delta \Theta \pi n d_{\mathrm{h}} \sin \Theta_{\mathrm{B}} / \lambda
$$

Here, $n$ is the index of refraction of the unexposed sample and $n_{1}$ is the index modulation amplitude, and the factor $\cos \left(2 \Theta_{B}\right)$ accounts for $p$-polarization. The solid line in Fig. 22 represents the fit of function Eq. (32) to the experimental data and yields an amplitude of the index modulation up to $n_{1}(785)=(-2.7 \pm 0.6) \cdot 10^{-4}$ and a hologram thickness of $d_{\mathrm{h}}=(0.62 \pm 0.07) \mathrm{mm}$.

Assuming that the electro-optic effect is the reason for the pronounced diffraction efficiency, an electric field must be present in addition to the spatial density modulation of small polarons. We now take into account that the light wave vectors propagate at a small angle with respect to the normal of the crystal and that the relation $r_{333} \approx 3 \cdot r_{113}$ (see e.g., Ref. 113) holds. Thereby, we approximate $n_{\text {eff }}$ with the index of refraction for extraordinary light polarization $n_{e}$ and $r_{\mathrm{eff}}$ $\approx r_{333}$. At wavelength of $\lambda=785 \mathrm{~nm}$, we find $n_{e}(785 \mathrm{~nm})$ $=(2.1776 \pm 0.0005){ }^{108}$ The electro-optic coefficient has been precisely determined to $r_{333}(632.8 \mathrm{~nm})=31.4 \mathrm{pm} / \mathrm{V}$ in Ref. 114. Together with the dispersive behavior published in Ref. 115 , we extrapolate $r_{333}(785 \mathrm{~nm})=30.8 \mathrm{pm} / \mathrm{V}$. All values hold for lithium niobate grown from the congruent melt

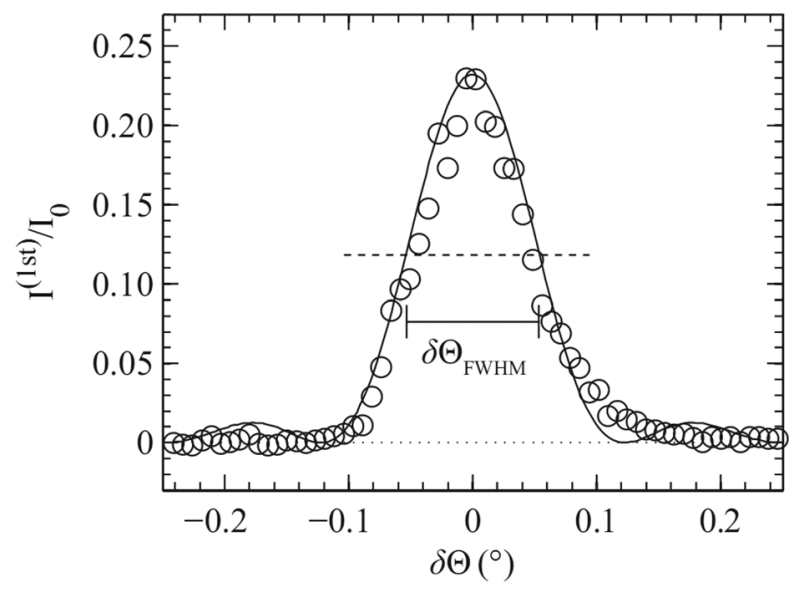

FIG. 22. Angular dependence of the intensity of the normalized first order diffracted beam $I^{(1 \mathrm{st})} / I_{0}$ at $t=1 \mu \mathrm{s}$ after the pump pulse as a function of the deviation $\delta \Theta$ of the Bragg-angle $\Theta_{\mathrm{B}}$. The solid line represents the result of fitting Eq. (32) to the experimental data. Reproduced with permission from Imlau et al., Opt. Express 19, 15322 (2011). Copyright 2011 OSA Publishing. 
and at room temperature. Then, the estimate for the electric field strength is $E=-2 \cdot n_{1} /\left(n_{3}^{3} \cdot r_{333}\right) \approx 17 \mathrm{kV} / \mathrm{cm}$.

Such a high electric field strength cannot be explained with the diffusion mechanism with a saturation field of $E_{\text {diff }}=\left(k_{\mathrm{B}} T / e\right)(2 \pi / \Lambda) \approx 1.25 \mathrm{kV} / \mathrm{cm}$. Here, $k_{\mathrm{B}}$ is the Boltzmann constant and $e$ the electron charge. We can also exclude drift mechanisms in the absence of an externally applied electric field. Instead, electric fields with a strength up to $100 \mathrm{kV} / \mathrm{cm}$ are common for Fe-doped lithium niobate induced via the bulk photovoltaic effect. ${ }^{23}$ Because of the absence of Fe-doping with significant concentration and a predominant valency $2+$ upon thermal reduction, a photovoltaic current may be assigned to the optical generation of small polarons in the samples under study.

A second remarkable feature of the recorded gratings is the shape of the temporal decay, which is non-exponential and best described by the phenomenological stretched exponential function (Eq. (18), solid line in Fig. 21). This observation cannot be assigned to holograms recorded conventionally via the photorefractive effect in doped lithium niobate that inevitably obey a single-exponential decay behavior. Furthermore, the corresponding lifetimes are determined by the dielectric relaxation time. ${ }^{116}$

In contrast, taking an electric field related to optically generated small bound polarons into account, a field decay according to the relaxation behavior of bound polaron densities must be expected. The latter is well-known to exhibit a nonexponential decay behavior upon exposure to single ns-laser pulses due to incoherent 3D hopping transport (see e.g., Refs. 15,67 , and 91) as it is the case for the samples under study. The decay lifetime of small bound $\mathrm{Nb}_{\mathrm{Li}}^{4+}$ polaron densities is thermally activated with an activation energy of $E_{\mathrm{a}}=(0.53 \pm 0.07) \mathrm{eV}$, and decays in the ms-range $(\tau=(2.8 \pm 0.4) \mathrm{ms})$. The coincidence with the corresponding data of the hologram relaxation dynamics in the same sample ( $\tau=(3.0 \pm 0.3) \mathrm{ms}$ and $\left.\bar{E}_{\mathrm{a}}=(0.57 \pm 0.07) \mathrm{eV}\right)$ is striking.

All these results thus strongly support the validity of bound-polaron-based bulk photovoltaic currents; thus, a relation between the optically generated small bound polaron density and the appearance of an electric field that alters the index of refraction via the linear electro-optic effect, seems likely. The underlying currents that result in the buildup of the electric field may be assigned to carriers from $\mathrm{Nb}_{\mathrm{Li}}^{4+}: \mathrm{Nb}_{\mathrm{Nb}}^{4+}$ bipolarons.

\section{IMPACT ON NONLINEAR PHOTONICS AND CONCLUSION}

This section addresses the question: What is the overall impact of the reviewed considerations and experimental findings? At a first glance, there is an obvious fundamental interest in defect physics, charge localization, non-equilibrium transport mechanism as well as light-matter-interaction on the nano- and ultrafast time scale beyond many other topics of solid state physics and optics from theory, experiment, and modeling. This is due to the fact that the small polaron problem contains a variety of modern physical questions and problems, some of them still to be solved, allowing for synergetic effects in research of different disciplines.
However, more striking is the impact of optical nonlinearities of small polarons in the field of nonlinear photonics. Nonlinear photonics strongly develops in the 21 st century and is accompanied by an increasing demand of nonlinear optical nano-materials with instantaneous and/or (ultra-)fast optical response. Here, $\mathrm{LiNbO}_{3}$ represents a promising candidate, and small polarons are at the origin of the interaction of (ultra-)short laser pulses with nanoscaled quasiparticles.

\section{A. Developing fields}

Optical nonlinearities of small polarons in particular, allow for the realization of some widely discussed, visionary applications, including three-dimensional, real-time holographic displays. The state-of-the-art systems use rerecordable photosensitive soft media, e.g., photo-addressable polymers, and allow for a proof-of-principle and the further improvement of these techniques. So far, however, the hologram recording time, the maximum efficiency, more precisely the sensitivity and dynamic range, as well as the possibilities for self-erasure of recorded holograms limit the transfer to commercial systems. Small-polaron based holography here may represent an important step towards realtime applications, as it is demonstrated by holographic image recording using a single ns-laser pulse and thermally reduced, nominally undoped $\mathrm{LiNbO}_{3}$ in Ref. 117. Figure 23 shows a photograph of the reconstructed image that appears for a moment, i.e., a duration of a few milliseconds, upon the ns-recording process. Since large pulse intensities are required for recording, the image is positioned within a $4 \mathrm{f}-$ optical setup of the signal beam, which also serves as a beam-clean up by Fourier filtering. According to the small polaron dynamics, the efficiency of the reconstructed image showed a temporal decay that can be modeled by a stretched-exponential function with characteristic decay time

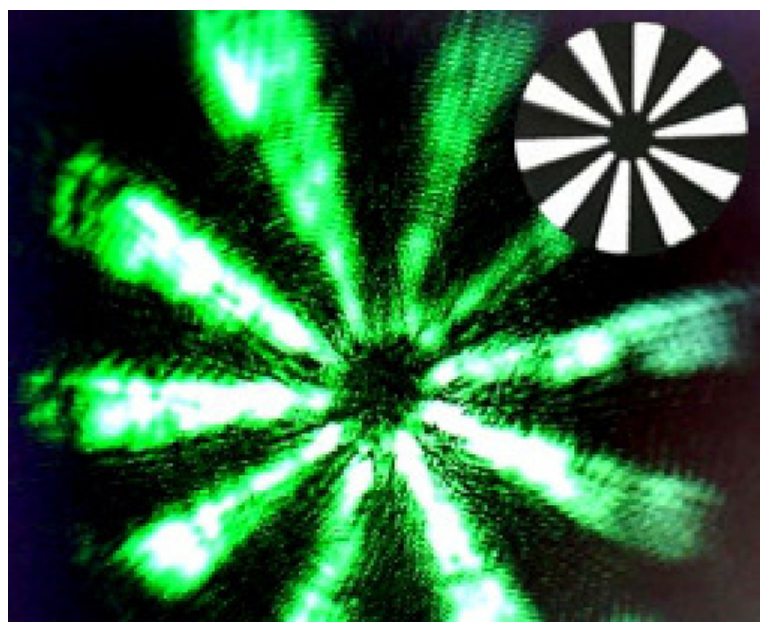

FIG. 23. Photograph of a reconstructed image recorded using a holographic setup with a ns-laser pulse $(532 \mathrm{~nm})$ and thermally reduced, nominally pure $\mathrm{LiNbO}_{3}$ crystals as hologram recording material. The image (a metal gobo, see inset) was placed within the signal beam and cleaned-up using a $4 \mathrm{f}$ optic. Recording was performed with a single $8 \mathrm{~ns}$ laser pulse (total pulse energy $\approx 22 \mathrm{~mJ}$ ). The image was photographed using a charge-coupled device (CCD) and an electromechanical shutter that opened the diffracted laser beam $1 \mathrm{~ms}$ after the laser pulse for a duration of $5 \mathrm{~ms}$. 
of $\approx 3 \mathrm{~ms}$. The recording mechanism follows the model approach presented in Section VI and is based on a spatial modulation of the optical dissociation of $\mathrm{Nb}_{\mathrm{Li}}^{4+}: \mathrm{Nb}_{\mathrm{Li}}^{4+}$ bipolarons. It is noteworthy that the millisecond decay represents self-erasure of the hologram; thus, further hologram recording becomes possible at a repetition rate of up to $100 \mathrm{~Hz}$, quite reasonable for display technologies. Even higher repetition rates become possible either by heating the crystal, i.e., acceleration of the incoherent hopping mechanism, or by using small free $\mathrm{Nb}_{\mathrm{Nb}}^{4+}$ polarons instead. A further advantage of this type of hologram recording is the possibility to record holograms with different colors in the blue, green (by means of $\mathrm{O}^{-}$or $\mathrm{Nb}_{\mathrm{Li}}^{4+}: \mathrm{Nb}_{\mathrm{Li}}^{4+}$ ), and red (by means of $\mathrm{Nb}_{\mathrm{Li}}^{4+}$ ) spectral range. At the same time, holograms can be reconstructed over a broad spectral range; both spectral dependencies of recording and reconstruction are important pre-requisites for color holography. Despite these promising features, $\mathrm{LiNbO}_{3}$ remains an expensive crystalline material, which may be applied in small-frame 3D-displays, such as for headmounted displays, or for applications in real-time interferometry, e.g., optical correlators for scanning fingerprints in the framework of security issues. Gamaly et al. used nominally undoped, stoichiometric $\mathrm{LiNbO}_{3}$ for the demonstration of 3D write-read-erase memory bits using 150 femtosecond laser pulses. ${ }^{111}$

A very recent holographic application based on the ultrafast, small polaron response time is described in Ref. 118 , where light-amplification of a weak signal beam is realized by means of wave-mixing with a strong pump. The underlying mechanism is the possibility to generate a light interference pattern even with beams of different color, i.e., frequency detuned beams, if pulses with a pulse duration in the sub-ps-time regime are chosen. As depicted in Fig. 24, amplification is observed in nominally undoped $\mathrm{LiNbO}_{3}$. A prerequisite of light amplification is either the possibility to record the fringe pattern, which is demonstrated in Section VI as well as in Ref. 118, and a significant nonlinearity in the recording/read-out process. Although the

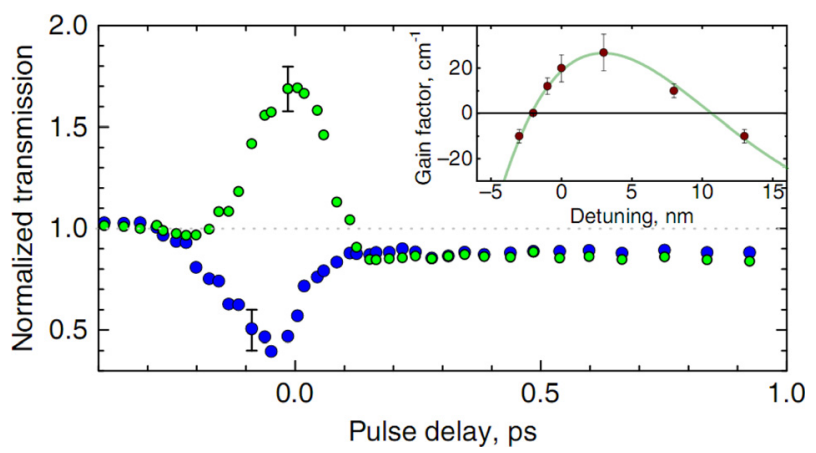

FIG. 24. Probe beam transmission versus time delay of the probe pulse with respect to the pump pulse in nominally undoped $\mathrm{LiNbO}_{3}$. The wavelengths of the probe pulse are $485 \mathrm{~nm}$ and $493 \mathrm{~nm}$ for blue and green dots, respectively, the pump wavelength is $\simeq 490 \mathrm{~nm}$. The inset shows the detuning dependence of the net gain factor (amplification minus absorption) for probe and pump pulses matched in time. Reproduced with permission from Odoulov et al., Nat. Commun. 6, 5866 (2015). Copyright 2015 Nature Publishing Group. Reproduced by permission of Nature Publishing Group. All rights reserved. underlying mechanism of the amplification process itself is not fully understood, so far, the role of small polarons cannot be excluded.

\section{B. Present applications}

Optical nonlinearities of small polarons need to be discussed in the framework of present applications of $\mathrm{LiNbO}_{3}$ in nonlinear photonics, as well. Very prominent is to use lithium niobate as a nonlinear optical crystal for frequency conversion. Here, small polarons limit the crystal's conversion efficiency, its operation lifetime, as well as the tolerable energy of the pump beam and the repetition rate of the pulse laser system itself, as it will be discussed in the following. It will be shown that, from a fundamental viewpoint, understanding of optical nonlinearities of small polarons has a large impact on optimizing converter features of $\mathrm{LiNbO}_{3}$. Examples include pre-treatment and/or doping with appropriate external dopants or tuning of the laser system parameters according to small polaron features.

Let us assume the generation of harmonic light pulses at $\lambda_{\mathrm{SHG}}=532 \mathrm{~nm}$ by pumping a nominally undoped $\mathrm{LiNbO}_{3}$ crystals with short, intense Gaussian laser pulses of a $Q$ switched YAG:Nd-laser operated at $\lambda_{\text {pump }}=1064 \mathrm{~nm}$ with intensity $I_{\text {pump }}$, repetition rate $f_{\text {rep }}$ and pulse durations in the nanosecond regime. Intensities of the generated harmonic pulses $I_{\mathrm{SHG}}$ exceeding $1 \mathrm{GW} / \mathrm{cm}^{2}$ at the output of the crystal are assumed. The aspect of quasi phase-matching, necessary for efficient frequency conversion in $\mathrm{LiNbO}_{3}$ will be discussed seperately below, as it is responsible for further pecularities of small polaron dynamics.

Regarding a first infrared laser pulse, free carrier excitation will occur in $\mathrm{LiNbO}_{3}$ by interband two-photon absorption due to the (self-)generated harmonic laser pulse at $532 \mathrm{~nm}$ (Section IV). The carrier density will be spatially limited to the beam path of the pulse and will increase towards the output surface of the crystal in accordance with the rise of the harmonic intensity. At the end of the ns-pulse a fraction of such carriers is trapped at $\mathrm{Nb}_{\mathrm{Nb}}$ and/or $\mathrm{Nb}_{\mathrm{Li}}$, giving rise to the appearance of a transient absorption. Here, we remind of their spectrally broad absorption features of the order of $\approx 1 \mathrm{eV}$ (cf. 5). In particular, the absorption related to small free $\mathrm{Nb}_{\mathrm{Nb}}^{4+}$ as well as to small bound $\mathrm{Nb}_{\mathrm{Li}}^{4+}$ polarons cover the wavelength of the laser pump beam at $1064 \mathrm{~nm}(1.165 \mathrm{eV})$. Phenomenologically, this effect is called green light-induced infrared absorption (GRIRA), ${ }^{119}$ a terminology that is common in laser physics to describe equivalent phenomena in the nonlinear optical crystal $\mathrm{KTiOPO}_{4}(\mathrm{KTP}){ }^{120}$ It must be added that also $\mathrm{O}^{-}$polarons are formed in this process, and that the absorption at $532 \mathrm{~nm}$ $(2.33 \mathrm{eV})$ is constituted by contributions of FP, GP, and HP. This transient absorption (or GRIRA), decays in a stretched exponential way with characteristic lifetimes in the microsecond $\left(\mathrm{Nb}_{\mathrm{Nb}}^{4+}\right)$ and millisecond $\left(\mathrm{Nb}_{\mathrm{Li}}^{4+}\right)$ regime as another small polaron feature in $\mathrm{LiNbO}_{3}$.

For a laser system in single pulse operation mode, there are no further consequences of GRIRA. However, in repetition mode, the appearance of GRIRA means that subsequent laser pulses will be strongly attenuated at both fundamental 
and harmonic wavelengths, if the time delay to the initial pulse falls within the small polaron lifetime $\tau_{\text {lifetime. In this }}$ case, absorption (i) suppresses the intensity of the harmonic wave, thus affecting the conversion efficiency, (ii) heats the crystal mainly by the interaction with the intense fundamental wave ${ }^{121}$ that (iii) results in the effect of thermal lensing via the thermo-optic effect. In case of very high intensities, even (iv) an irreversible thermo-mechanical damage of the crystal must be expected. From the viewpoint of a laser system with intra-cavity frequency conversion, GRIRA furthermore represents an increase of laser-cavity losses and, depending on the magnitude of the absorption change, may result in the complete break-down of laser operation.

Besides, the generation of small polarons in the beam path of the laser pulses is accompanied with changes in the index of refraction (Section VI) that are a second source for laser break-down in intra-cavity setups. The index changes exhibit a spatial profile transversal to the beam path that is determined by the spatial (Gaussian) intensity profile of the laser pulses and act as a focusing or de-focusing index lens depending on the sign of the index change. This effect results in pronounced fanning, e.g., of femtosecond laser pulses in $\mathrm{LiNbO}_{3}{ }^{122}$ and has a similar effect on the laser systems operation as the so-called optical damage that has been studied intensively in the framework of the photorefractive effect in $\mathrm{LiNbO}_{3}$ particularly with the aim to reduce its action by stoichiometry. ${ }^{123-127}$ In contrast, index changes based on optical nonlinearities of small-polarons are short-lived and recover on a millisecond time scale.

In order to overcome such manifold problems related with GRIRA, the repetition rate $f_{\text {rep }}$ of the laser system must be chosen such that small polarons completely relax between two subsequent laser pulses, i.e., $f_{\text {rep }} \leq 1 / \tau_{\text {lifetime. }}$. If higher repetition rates are required, one may take advantage of the hopping process, mainly responsible for the magnitude of the decay time of the transient absorption and being thermally activated. For this purpose, a $\mathrm{LiNbO}_{3}$ crystal simply needs to be operated at elevated temperatures. Here, care must be taken not to activate further unwanted phenomena such as proton transport.

The general tendency in laser physics, however, is to completely suppress the appearance of transient optical nonlinearities, i.e., to suppress the formation of small polarons at all. This can be realized for small bound polarons by removing the related intrinsic (or extrinsic) defect centers. For $\mathrm{LiNbO}_{3}$, appropriate techniques have been well developed over the last decades. One possibility is to use stoichiometric (or near-stoichiometric) $\mathrm{LiNbO}_{3}$ crystals (reviewed, e.g., in Ref. 39). Or, $\mathrm{Nb}_{\mathrm{Li}}$ antisites can be removed by doping of $\mathrm{LiNbO}_{3}$ with optically not active extrinsic defects that are incorporated in the crystal lattice on a $\mathrm{Li}$ site such as $\mathrm{Mg}_{\mathrm{Li}}$ or $\mathrm{Y}_{\mathrm{Li}}$ (reviewed, e.g., in Ref. 127). Such additional dopants can be found in most of the commercial $\mathrm{LiNbO}_{3}$ frequency converters. In both cases, however, small free polarons are not removed due to their structural relation with the undisturbed $\mathrm{Nb}_{\mathrm{Nb}}$ crystal sub-lattice, so that GRIRA and causally related index changes remain (contrary to photovoltaic driven effects). Nevertheless, the spectral features of GRIRA as well as its dynamics changes significantly, as it is demonstrated in the transient absorption study of Conradi et al. ${ }^{128}$ for $\mathrm{LiNbO}_{3}$ with different $\mathrm{Mg}_{\mathrm{Li}}$ dopant concentrations in the VIS and by Kitaeva et al. up to the infrared. ${ }^{129}$ For the case of frequency conversion using ultrafast laser systems, the formation of small polarons was studied in $\mathrm{MgO}$-doped crystals in Ref. 130. As the pulse duration may fall below the small polaron formation time of $\approx 100 \mathrm{fs}$, however, the interaction with free-carrier absorption (FCA) much larger than small polaron absorption needs to be considered. ${ }^{131}$ The FCA magnitude is higher due to a comparable large number density of free carriers (below $1 \%$ of free carriers are trapped as small polarons, see also Table I) as well as larger absorption cross section. ${ }^{84}$

We note that the interaction of fundamental and harmonic pulses with $\mathrm{Nb}_{\mathrm{Nb}}^{4+}: \mathrm{Nb}_{\mathrm{Li}}^{4+}$ bipolarons can be discussed in the same manner by considering optical dissociation of $\mathrm{BP}$, and formation of FP and HP. Although it seems that thermally reduced $\mathrm{LiNbO}_{3}$ may not be considered for frequency conversion, the appearance of a transient transparency due to BP dissociation and particularly at the wavelength of the harmonic may be of interest. Merschjann et al. were able to demonstrate that, for a so-called moderate thermal pre-treatment of $\mathrm{LiNbO}_{3}$, it becomes possible to completely suppress the appearance of a transient, i.e., of GRIRA. In this case, however, the BP groundstate absorption is to be considered for the initial pulse, which is comparably weak at $1064 \mathrm{~nm}$ and may be less critical if the nonlinear optical crystal is placed externally to the laser cavity.

So far, the appearance of GRIRA is discussed without consideration of the ferroelectric domain structure in $\mathrm{LiNbO}_{3}$. For efficient frequency conversion, i.e., in order to make use of the larger nonlinear optical coefficients of $\mathrm{LiNbO}_{3}$, quasi phase matching is applied. ${ }^{132,133}$ For this purpose, a periodic inversion of the direction of spontaneous polarization is required that is realized by periodic electricfield poling of the crystal bulk. ${ }^{134}$ Even at elevated temperature, however, this remains challenging taking into account the huge coercitive field of several $\mathrm{kV} / \mathrm{mm}$ of $\mathrm{LiNbO}_{3}$ from the congruently melt. As a result, thin slabs of periodically poled $\mathrm{LiNbO}_{3}$ (PPLN) are commercially available that are well suitable as frequency converters in small-sized, lowpower laser systems. An interesting approach for the synthesis of bulk PPLN, based on temperature fluctuations during Czochralski growth, is presented by Feng et al. ${ }^{135}$ Periodic temperature fluctuations may be induced by displacing the rotation axis from the symmetry axis of the temperature field. In order to accentuate the growth striations produced, the melt is doped with yttrium, $\mathrm{Y}_{\mathrm{Li}}$. From the boule, PPLN crystals with thicknesses of several millimeter can be successfully prepared, and it is shown by Naumova et al. that further dopants can be applied. ${ }^{136}$

As a specific feature of this crystal growth technique, stoichiometry and concentration of $\mathrm{Y}_{\mathrm{Li}}$ are spatially modulated in direction of the propagating laser pulses: $;{ }^{137}$ thus, the type of pulse-induced small polarons and respective number densities are expected to vary significantly. It becomes rather difficult to estimate the interplay of optical effects of the different regimes and to predict overall pulse propagation. 
Nevertheless, the comprehensive knowledge of optical nonlinearities of small polarons obtained for $\mathrm{LiNbO}_{3}$, so far (cf. Section III), allows for the modeling, e.g., of the dynamics of the transient absorption/GRIRA, in PPLN:Y. A respective comprehensive study is presented by Schoke et al. in Ref. 138. Figure 25 highlights the temporal evolution of the lightinduced absorption in a PPLN:Y crystal in direct comparison to single-domain, nominally pure $\mathrm{LiNbO}_{3}$. The crystals have been thermally pre-treated to probe the stoichiometry alteration by means of a $\mathrm{Nb}_{\mathrm{Li}}^{4+}: \mathrm{Nb}_{\mathrm{Nb}}^{4+}$ bipolaron modulation. For the PPLN:Y sample, a transient transparency at $488 \mathrm{~nm}$ initially induced by the pump pulse changes to a transient absorption at $1 \mathrm{~ms}$ prior to its decay ( $\tau>0.1 \mathrm{~s}$ ). Obviously, this behavior is a result of two super-imposed decay components with different time constants and opposite sign in their amplitudes. The key in understanding this behavior are the different contributions from different layers of the PPLN crystal to the overall signal: congruent layers contribute mainly by $\mathrm{Nb}_{\mathrm{Li}}$ defects, particularly by the bipolaron behavior, which is responsible for a light-induced transparency in the blue spectral range and a short recombination time due to a high antisite concentration in such layers. (Near-)stoichiometric layers account for hole polaron properties, i.e., a light-induced absorption in the blue spectral range and a comparably long lifetime. Hence, the deconvolution of the signal in two components according to the inset of Fig. 25 essentially indicates the respective contributions of bipolaron recombination and hole polaron relaxation to the overall signal.

We like to note that all these considerations are not only valid for the nonlinear optical crystal $\mathrm{LiNbO}_{3}$. Polaronic effects are found in nearly any nonlinear optical crystal and are discussed at the origin of the so-called blue light-induced infrared absorption (BLIIRA) in $\mathrm{KNbO}_{3},{ }^{46,139}$ GRIRA $^{120}$ or gray tracking in $\mathrm{KTiOPO}_{4}{ }^{48,140,141}$ and in $\mathrm{LiTaO}_{3}{ }^{47}$ Furthermore, polarons limit the performance of $\mathrm{LiB}_{3} \mathrm{O}_{5}$ $(\mathrm{LBO})^{49}$ and $\beta-\mathrm{BaB}_{2} \mathrm{O}_{4}(\mathrm{BBO}),{ }^{45}$ which are technologically of utmost importance for high-power laser applications. Even the surface growth of amorphous Si on LBO crystals is

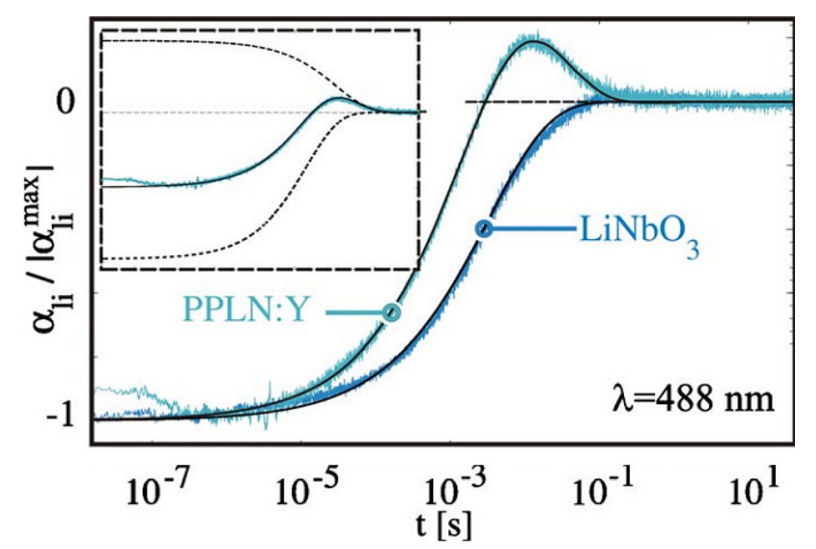

FIG. 25. Transient absorption in periodically poled $\mathrm{LiNbO}_{3}$ and reduced single-domain nominally pure lithium niobate probed at $\lambda=488 \mathrm{~nm}, I_{\mathrm{P}}=$ $800 \mathrm{GW} / \mathrm{m}^{2}$. Insets: complete plots for PPLN:Y according to a superposition of two stretched exponentials given by Eq. (18) (solid line), with its single components (lines). Reproduced with permission from Schoke et al., Phys. Rev. B 81, 132301 (2010). Copyright 2010 APS Publishing. discussed to originate from small polarons that are charging the exposed surface area. ${ }^{142}$ Studies of optical nonlinearities of small polarons are supported in any of these materials by means of electron-spin resonance at low temperatures. ${ }^{43,44}$

In conclusion, the exciting interplay between optical nonlinearities commonly assigned to continuum systems on the one side and small polarons that represent localities on the other side is surveyed. The key in closing the gap between continuum and locality is either the appearance of optical excitation from small polarons to energy levels that are resonant with bandlike states as well as the observation of ensembles of small polarons with large number density. It is well demonstrated-and without any doubt-that the small polaron approach is very powerful to describe the linear and nonlinear optical properties of $\mathrm{LiNbO}_{3}$. It particularly satisfies the demands for the understanding of processes on the atomic length and ultrafast time scale in the age of nanoscience and femtonics with appropriate techniques widely available for investigations. An outstanding strength is the all-over modeling, e.g., the broad-band, asymmetric absorption bands, and of transport issues, e.g., the lowmobility incoherent current density, and of the possibility to assign tensorial properties of the photo-galvanic effect with the atomic structure of $\mathrm{LiNbO}_{3}$ and of carrier dynamics over several magnitudes of time, i.e., from femtoseconds to seconds. Moreover, any of the (phenomenologically) wellknown effects, e.g., the bulk photovoltaic effect, GRIRA, light-induced index changes, and many more, can be explained comprehensively. It is the basis for predictions and material tuning and/or material engineering in the framework of existing - as well as visionary - applications in the field of nonlinear photonics. Although a small polaron theory is developed in a convincing way, a variety of experimental studies particularly on the nanoscale and in the ultrafast time domain are expected. This will further raise the impact of optical nonlinearities of small polarons in $\mathrm{LiNbO}_{3}$.

\section{ACKNOWLEDGMENTS}

The authors thank Ortwin Schirmer for frutiful discussions, Gabor Corradi for fruitful discussions and $\mathrm{LiNbO}_{3}$ samples for holographic measurements, Bettina Schoke, Stefan Torbrügge, and Sarah Masch for fruitful discussion and experimental support, Gerda Cornelsen and Werner Geisler for sample preparation and the Deutsche Forschungsgemeinschaft (Project Nos. IM37/5, INST 190/ 137-1 FUGG, and INST 160/165-1 FUGG) and the Deutscher Akademischer Austausch Dienst in cooperation with the Hungarian Scholarship Board Office (Project Nos. 50445542, 54377942, and 57139940) for financial support.

${ }^{1}$ D. Emin, Polarons (Cambridge University Press, Cambridge, 2013).

${ }^{2}$ D. Emin, Polarons, 10th ed. (McGraw Hill Encyclopedia of Science and Technology, 2007), Vol. 14, p. 125.

${ }^{3}$ P. Calvani, "Optical properties of polarons," Riv. Nuovo Cimento 24, 1-71 (2001).

${ }^{4}$ O. F. Schirmer, M. Imlau, C. Merschjann, and B. Schoke, "Electron small polarons and bipolarons in $\mathrm{LiNbO}_{3}$," J. Phys.: Condens. Matter. 21, 123201 (2009).

${ }^{5} \mathrm{O}$. F. Schirmer, "O $\mathrm{O}^{-}$bound small polarons in oxide materials," J. Phys.: Condens. Matter 18, R667 (2006). 
${ }^{6}$ D. Emin, "Phonon-assisted transition rates I. Optical-phonon-assisted hopping in solids," Adv. Phys. 24, 305 (1975).

${ }^{7}$ D. Emin, "Optical properties of large and small polarons and bipolarons," Phys. Rev. B 48, 13691 (1993).

${ }^{8}$ S. Redfield and W. J. Burke, "Optical absorption edge of $\mathrm{LiNbO}_{3}$," J. Appl. Phys. 45, 4566 (1974).

${ }^{9}$ D. Berben, K. Buse, S. Wevering, P. Herth, M. Imlau, and T. Woike, "Lifetime of small polarons in iron-doped lithium-niobate crystals," J. Appl. Phys. 87, 1034 (2000).

${ }^{10}$ J. Shi, H. Fritze, G. Borchardt, and K. D. Becker, "Defect chemistry, redox kinetics, and chemical diffusion of lithium deficient lithium niobate," Phys. Chem. Chem. Phys. 13, 6925 (2011).

${ }^{11}$ K. L. Sweeney and L. E. Halliburton, "Oxygen vacancies in lithium niobate," Appl. Phys. Lett. 43, 336 (1983).

${ }^{12}$ F. Jermann and J. Otten, "Light-induced charge transport in $\mathrm{LiNbO}_{3}: \mathrm{Fe}$ at high light intensities,” J. Opt. Soc. Am. B 10, 2085 (1993).

${ }^{13}$ O. Beyer, D. Maxein, T. Woike, and K. Buse, "Generation of small bound polarons in lithium niobate crystals on the subpicosecond time scale," Appl. Phys. B 83, 527 (2006).

${ }^{14}$ P. Herth, T. Granzow, D. Schaniel, T. Woike, M. Imlau, and E. Krätzig, "Evidence for light-induced hole polarons in $\mathrm{LiNbO}_{3}$," Phys. Rev. Lett. 95, 067404 (2005).

${ }^{15}$ C. Merschjann, D. Berben, M. Imlau, and M. Wöhlecke, "Evidence for two-path recombination of photoinduced small polarons in reduced $\mathrm{LiNbO}_{3}$," Phys. Rev. Lett. 96, 186404 (2006).

${ }^{16}$ Y. Qiu, K. B. Ucer, and R. T. Williams, "Formation time of a small electron polaron in $\mathrm{LiNbO}_{3}$ : Measurements and interpretation," Phys. Status Solidi C 2, 232 (2005).

${ }^{17}$ S. Sasamoto, J. Hirohashi, and S. Ashihara, "Polaron dynamics in lithium niobate upon femtosecond pulse irradiation: Influence of magnesium doping and stoichiometry control," J. Appl. Phys. 105, 083102 (2009).

${ }^{18}$ J. Carnicero, M. Carrascosa, G. García, and F. Agulló-López, "Site correlation effects in the dynamics of iron impurities $\mathrm{Fe}^{2+} / \mathrm{Fe}^{3+}$ and antisite defects $\mathrm{Nb}_{\mathrm{Li}}^{4+} / \mathrm{Nb}_{\mathrm{Li}}^{5+}$ after a short-pulse excitation in $\mathrm{LiNbO}_{3}$," Phys. Rev. B 72, 245108 (2005)

${ }^{19}$ C. Merschjann, M. Imlau, H. Brüning, B. Schoke, and S. Torbrügge, "Nonexponential relaxation dynamics of localized carrier densities in oxide crystals without structural or energetic disorder," Phys. Rev. B 84, 052302 (2011).

${ }^{20}$ D. C. Hutchings, M. Sheik-Bahae, D. J. Hagan, and E. W. Van Stryland, "Kramers-Krönig relations in nonlinear optics," Opt. Quantum Electron 24, 1 (1992).

${ }^{21}$ Kramers-Kronig Relations in Optical Materials Research, edited by V. Lucarini, J. J. Saarinen, K. E. Peiponen, and E. M. Vartiainen (Springer Verlag, 2005).

${ }^{22}$ O. F. Schirmer, M. Imlau, and C. Merschjann, "Bulk photovoltaic effect of $\mathrm{LiNbO}_{3}: \mathrm{Fe}$ and its small-polaron-based microscopic interpretation," Phys. Rev. B 83, 165106 (2011).

${ }^{23}$ A. M. Glass, D. von der Linde, and T. J. Negran, "High-voltage bulk photovoltaic effect and the photorefractive process in $\mathrm{LiNbO}_{3}$," Appl. Phys. Lett. 25, 233 (1974).

${ }^{24}$ G. Chanussot and A. M. Glass, "A bulk photovoltaic effect due to electron-phonon coupling in polar crystals," Phys. Lett. A 59, 405 (1976).

${ }^{25}$ W. Jösch, R. Munser, W. Ruppel, and P. Würfel, "The photovoltaic effect and the charge transport in $\mathrm{LiNbO}_{3}$," Ferroelectricity 21, 623 (1978).

${ }^{26} \mathrm{~V}$. M. Fridkin, "Review of recent work on the bulk photovoltaic effect in ferro- and piezoelectrics," Ferroelectrics 53, 169 (1984).

${ }^{27}$ F. el Guibaly, "Models for the bulk photovoltaic effect in ferroelectric crystals," Can. J. Phys. 66, 649 (1988).

${ }^{28} \mathrm{~K}$. Buse, "Light-induced charge transport processes in photorefractive crystals I: Models and experimental methods," Appl. Phys. B 64, 273-291 (1997).

${ }^{29} \mathrm{~K}$. Buse, "Light-induced charge transport processes in photorefractive crystals II: Materials," Appl. Phys. B 64, 391-407 (1997).

${ }^{30}$ A. M. Glass, "The photorefractive effect," Opt. Eng. 17, 175470 (1978).

${ }^{31}$ E. Krätzig and H. Kurz, "Photorefractive and photovoltaic effects in doped $\mathrm{LiNbO}_{3}$," Opt. Acta 24, 475 (1977).

${ }^{32}$ B. I. Sturman and V. M. Fridkin, The Photovoltaic and Photorefractive Effects in Noncentrosymmetric Materials (Clarendon, Oxford, 1996).

${ }^{33}$ K. Buse, J. Imbrock, E. Kratzig, and K. Peithmann, "Photorefractive effects in $\mathrm{LiNbO}_{3}$ and LiTaO3," in Photorefractive Materials and their Applications II, Number 62 in Topics in Applied Physics, edited by P. Günter and J. P. Huignard (Springer-Verlag, 1989), p. 83.
${ }^{34}$ Photorefractive Materials and Their Applications I, II and II, edited by P. Günter and J. P. Huignard (Springer-Verlag, Heidelberg 1988, 1989), Vol. 61-62.

${ }^{35}$ M. Imlau, H. Brüning, B. Schoke, R. S. Hardt, D. Conradi, and C. Merschjann, "Hologram recording via spatial density modulation of $\mathrm{Nb}_{\mathrm{Li}}^{4+/ 5+}$ antisites in lithium niobate," Opt. Express 19, 15322-15338 (2011).

${ }^{36}$ H. Bruening, V. Dieckmann, B. Schoke, K. M. Voit, M. Imlau, G. Corradi, and C. Merschjann, "Small-polaron based holograms in $\mathrm{LiNbO}_{3}$ in the visible spectrum," Opt. Express 20, 13326-13336 (2012).

${ }^{37}$ O. Beyer, D. Maxein, K. Buse, B. Sturman, H. Hsieh, and D. Psaltis, "Femtosecond time-resolved absorption processes in lithium niobate crystals," Opt. Lett. 30, 1366-1368 (2005).

${ }^{38}$ L. Arizmendi, "Photonic applications of lithium niobate crystals," Phys. Status Solidi A 201, 253 (2004).

${ }^{39}$ H. Hatano and Y. K. K. Liu, "Growth and photorefractive properties of stoichiometric $\mathrm{LiNbO}_{3}$ and LiTaO3," in Photorefractive Materials and their Applications II, Number 62 in Topics in Applied Physics, edited by P. Günter and J. P. Huignard (Springer-Verlag, 1989), p. 127.

${ }^{40}$ T. Volk and M. Wöhlecke, Lithium Niobate-Defects, Photorefraction and Ferroelectric Switching (Springer, Berlin, 2008).

${ }^{41}$ L. Hesselink, S. S. Orlov, A. Lie, A. Akella, D. Lande, and R. R. Neurgaonkar, "Photorefractive materials for nonvolatile volume holographic data storage," Science 282, 1089 (1998).

${ }^{42}$ K. Buse, A. Adibi, and D. Psaltis, "Non-volatile holographic storage in doubly doped lithium niobate crystals," Nature 393, 665 (1998).

${ }^{43}$ L. E. Halliburton, N. C. Giles, and T. H. Myers, "Development of nonlinear optical materials for optical parametric oscillator and frequency conversion applications in the near- and mid-infrared," Final Technical Report No. A342373, 2003.

${ }^{44}$ M. M. Chirila, N. Y. Garces, L. E. Halliburton, S. G. Demos, T. A. Land, and H. B. Radousky, "Production and thermal decay of radiation-induced point defects in $\mathrm{KD}_{2} \mathrm{PO}_{4}$ crystals," J. Appl. Phys. 94, 6456 (2003).

${ }^{45}$ W. Hong, L. E. Halliburton, K. T. Stevens, D. Perlov, G. C. Catella, R. K. Route, and R. S. Feigelson, "Electron paramagnetic resonance study of electron and hole traps in $\beta-\mathrm{BaB}_{2} \mathrm{O}_{4}$ crystals," J. Appl. Phys. 94, 2510-2515 (2003).

${ }^{46}$ M. Zgonik, M. Ewart, C. Medrano, and P. Günter, Photorefractive Effects in $\mathrm{KNbO}_{3}$ (Springer, New York, 2007).

${ }^{47} \mathrm{~S}$. Wevering, J. Imbrock, and E. Krätzig, "Relaxation of light-induced absorption changes in photorefractive lithium tantalate crystals," J. Opt. Soc. Am. B 18, 472 (2001).

${ }^{48}$ G. J. Edwards, M. P. Scripsick, L. E. Halliburton, and R. F. Belt, "Identification of radiation-induced hole center in $\mathrm{KTiOPO}_{4}$," Phys. Rev. B 48, 6884-6891 (1993).

${ }^{49}$ W. Hong, M. M. Chirila, N. Y. Garces, L. E. Halliburton, D. Lupinski, and P. Villeval, "Electron paramagnetic resonance and electron-nuclear double resonance study of trapped-hole centers in $\mathrm{LiB}_{3} \mathrm{O}_{5}$ crystals," Phys. Rev. B 68, 094111 (1993).

${ }^{50}$ T. Holstein, "Studies of polaron motion: 1 . The molecular crystal model," Ann. Phys. 8, 325 (1959).

${ }^{51}$ L. D. Landau, "Über die Bewegung der Elektronen im Kristallgitter," Phys. Z. Sowjetunion 3, 664-665 (1933).

${ }^{52}$ T. Holstein, "Studies of polaron motion: Part II. The 'small' polaron," Ann. Phys. 8, 343 (1959).

${ }^{53}$ S. I. Pekar, Sov. Phys. JETP 16, 335 (1946).

${ }^{54}$ I. G. Austin and N. F. Mott, "Polarons in crystalline and non-crystalline materials," Adv. Phys. 18, 41 (1969).

${ }^{55}$ J. Appel, "Polarons," in Solid State Physics, edited by F. Seitz, D. Turnbull, and H. Ehrenreich (Academic Press-New York and London, 1968), Vol. 21, Chap. III, pp. 193-391.

${ }^{56} \mathrm{D}$. Emin, "Pair breaking in semiclassical singlet small-bipolaron hopping," Phys. Rev. B 53, 1260-1268 (1996).

${ }^{57}$ B. Faust, H. Müller, and O. F. Schirmer, "Free small polarons in $\mathrm{LiNbO}_{3}$," Ferroelectrics 153, 297 (1994).

${ }^{58}$ O. F. Schirmer, O. Thiemann, and M. Wöhlecke, "Defects in $\mathrm{LiNbO}_{3}-\mathrm{I}$. Experimental aspects," J. Phys. Chem. Solids 52, 185 (1991).

${ }^{59}$ J. L. Ketchum, K. L. Sweeney, L. E. Halliburton, and A. F. Armington, "Vacuum annealing effects in lithium niobate," Phys. Lett. A 94, 450 (1983).

${ }^{60}$ D. M. Smyth, "Defects and transport in $\mathrm{LiNbO}_{3}$," Ferroelectrics 50, 93 (1983).

${ }^{61}$ O. F. Schirmer, S. Juppe, and J. Koppitz, "ESR-, optical and photovoltaic studies of reduced undoped $\mathrm{LiNbO}_{3}$," Cryst. Latt. Defects Amorphous Mater. 16, 353 (1987). 
${ }^{62}$ O. F. Schirmer, H. J. Reyher, and M. Wöhlecke, "Characterization of point defects in photorefractive oxide crystals by paramagnetic resonance methods," in Insulating Materials for Optoelectronics-New Developments, edited by F. Agulló-López (World Scientific Publishing, 1995), pp. 93-124.

${ }^{63}$ I. S. Akhmadullin, V. A. Golenishchev-Kutuzov, and S. A. Migachev, "Electronic structure of deep centers in $\mathrm{LiNbO}_{3}$," Phys. Solid State 40, 1012-1018 (1998).

${ }^{64}$ Y. Li, W. G. Schmidt, and S. Sanna, "Intrinsic $\mathrm{LiNbO}_{3}$ point defects from hybrid density functional calculations," Phys. Rev. B 89, 174106 (2014).

${ }^{65}$ Y. Li, S. Sanna, and W. G. Schmidt, "Modeling intrinsic defects in $\mathrm{LiNbO}_{3}$ within the Slater-Janak transition state model," J. Chem. Phys. 140, 234113 (2014).

${ }^{66}$ J. Koppitz, O. F. Schirmer, and A. I. Kuznetsov, "Thermal dissociation of bipolarons in reduced undoped $\mathrm{LiNbO}_{3}$," Europhys. Lett. 4, 1055 (1987).

${ }^{67}$ P. Herth, D. Schaniel, T. Woike, T. Granzow, M. Imlau, and E. Krätzig, "Polarons generated by laser pulses in doped $\mathrm{LiNbO}_{3}$," Phys. Rev. B 71, 125128 (2005)

${ }^{68}$ O. F. Schirmer and D. von der Linde, "Two-photon and X-ray-induced $\mathrm{Nb}^{4+}$ and $\mathrm{O}^{-}$small polarons in $\mathrm{LiNbO}_{3}$," Appl. Phys. Lett. 33, 35 (1978).

${ }^{69}$ F. Jermann, M. Simon, R. Böwer, E. Krätzig, and O. F. Schirmer, "Light-induced absorption changes in reduced lithium niobate," Ferroelectrics 165, 319 (1995)

${ }^{70}$ K. Buse, F. Jermann, and E. Krätzig, "Two-step photorefractive hologram recording in $\mathrm{LiNbO}_{3}: \mathrm{Fe}, "$ Ferroelectrics 141, 197 (1993).

${ }^{71}$ P. Nagels, "Experimental Hall effect data for a small-polaron semiconductor," in The Hall Effect and its Applications, edited by C. L. Chien and C. R. Westlake (Plenum Press, New York, 1980), pp. 253-280.

${ }^{72}$ A. Alexandrov and J. Ranninger, "Theory of bipolarons and bipolaronic bands," Phys. Rev. B 23, 1796-1801 (1981).

${ }^{73}$ L. E. Halliburton, K. L. Sweeney, and C. Y. Chen, "Electron spin resonance and optical studies of point defects in lithium niobate," Nucl. Instrum. Methods Phys. Res. B 1, 344 (1984).

${ }^{74}$ L. Arizmendi, J. M. Cabrera, and F. Agullo-Lopez, "Defects induced in pure and doped $\mathrm{LiNbO}_{3}$ by irradiation and thermal reduction," J. Phys. C: Solid State Phys. C17, 515 (1984)

${ }^{75}$ H. G. Reik and D. Heese, "Frequency dependence of the electrical conductivity of small polarons for high and low temperatures," J. Phys. Chem. Solids 28, 581-596 (1967).

${ }^{76} \mathrm{M}$. Klinger, "Quantum theory of non-steady-state conductivity in low mobility solids,” Phys. Lett. 7, 102-104 (1963).

${ }^{77}$ C. Merschjann, B. Schoke, D. Conradi, M. Imlau, G. Corradi, and K. Polgar, "Absorption cross sections and number densities of electron and hole polarons in congruently melting $\mathrm{LiNbO}_{3}$," J. Phys.: Condens. Matter 21, 015906 (2009).

${ }^{78}$ J. Koppitz, O. F. Schirmer, M. Wöhlecke, A. I. Kuznetsov, and B. C. Grabmaier, "Threshold effects in $\mathrm{LiNbO}_{3}: \mathrm{Mg}$ caused by change of electron-lattice coupling," Ferroelectrics 92, 233-241 (1989).

${ }^{79}$ G. Montemezzani, P. Rogin, M. Zgonik, and P. Günter, "Interband photorefractive effects in $\mathrm{KNbO}_{3}$ induced by ultraviolet illumination," Opt. Lett. 18, 1144-1146 (1993).

${ }^{80} \mathrm{G}$. Montemezzani, P. Rogin, M. Zgonik, and P. Günter, "Interband photorefractive effects-Theory and experiments in $\mathrm{KNbO}_{3}$," Phys. Rev. B 49, 2484-2502 (1994).

${ }^{81}$ H. Yochum, K. Ucer, R. Williams, P. Sheldon, V. Nagirnyi, V. Denks, L. Grigorjeva, D. Millers, and E. Kotomin, "Short-pulse excitation and spectroscopy of $\mathrm{KNbO}_{3}, \mathrm{LiNbO}_{3}$ and $\mathrm{KTiOPO}_{4}$," in 8th Europhysical Conference on Defects in Insulating Materials (EURODIM98), Univ. Keele, Keele, England, Jul. 06-11, 1998; [Radiat. Eff. Defects Solids 150, 271-276 (1999)].

${ }^{82}$ O. Beyer, D. Maxein, K. Buse, B. Sturman, H. Hsieh, and D. Psaltis, "Investigation of nonlinear absorption processes with femtosecond light pulses in lithium niobate crystals," Phys. Rev. E 71, 056603 (2005).

${ }^{83}$ H. Badorreck, "Aufbau und charakterisierung eines fs-spektroskopieexperiments im UV/VIS/NIR," Masterarbeit (Osnabrueck University, 2011).

${ }^{84}$ H. Badorreck, S. Nolte, F. Freytag, and M. Imlau, "Two-photon absorption in presence of small polaron formation in lithium niobate," Proceedings, Photorefractive Photonics (PR'15), Villars, Switzerland, 2015.

${ }^{85}$ R. Kohlrausch, "Über das Dellmann'sche Elektrometer," Ann. Phys. Chem. 148, 353-405 (1847).

${ }^{86} \mathrm{G}$. Williams and D. C. Watts, "Non-symmetrical dielectric relaxation behaviour arising from a simple empirical decay function," Trans. Faraday Soc. 66, 80 (1970).
${ }^{87}$ F. Alvarez, A. Alegra, and J. Colmenero, "Relationship between the timedomain Kohlrausch-Williams-Watts and frequency-domain HavriliakNegami functions," Phys. Rev. B 44, 7306-7312 (1991).

${ }^{88}$ B. Sturman, E. Podivilov, and M. Gorkunov, "Origin of stretched exponential relaxation for hopping-transport models," Phys. Rev. Lett. 91, 176602 (2003).

${ }^{89} \mathrm{C}$. Merschjann, "Optically generated small polarons: Time-resolved pump-multiprobe experiments in lithium niobate vs. random walk charge transport simulations in oxide crystals," Ph.D. dissertation (Universität Osnabrück, 2007).

${ }^{90}$ L. Guilbert, "Swiss cheese model," private communication (2014).

${ }^{91}$ C. Merschjann, B. Schoke, and M. Imlau, "Influence of chemical reduction on the particular number densities of light-induced small electron and hole polarons in nominally pure $\mathrm{LiNbO}_{3}$," Phys. Rev. B 76, 085114 (2007).

${ }^{92}$ S.-M. Bahae, A. A. Said, T. H. Wei, D. J. Hagan, and E. W. Van Stryland, "Sensitive measurement of optical nonlinearities using a single beam," IEEE J. Quantum Electron. 26, 760 (1990).

${ }^{93}$ M. Woehlecke, G. Corradi, and K. Betzler, "Optical methods to characterise the composition and homogeneity of lithium niobate single crystals," Appl. Phys. B 63, 323 (1996).

${ }^{94}$ A. Seilmeier and W. Kaiser, "Generation of tunable picosecond light pulses covering the frequency range between 2700 and $32,000 \mathrm{~cm}^{-1}$," Appl. Phys. A 23, 113 (1980).

${ }^{95} \mathrm{H}$. Li, F. Zhou, X. Zhang, and W. Ji, "Picosecond Z-scan study of bound electronic Kerr effect in $\mathrm{LiNbO}_{3}$ crystal associated with two-photon absorption," Appl. Phys. B 64, 659 (1997).

${ }^{96}$ R. DeSalvo, A. Said, D. Hagan, E. Van Stryland, and M. Sheik-Bahae, "Infrared to ultraviolet measurements of two-photon absorption and $n_{2}$ in wide bandgap solids,” IEEE J. Quantum Electron 32, 1324 (1996).

${ }^{97}$ R. Ganeev, I. Kulagin, A. Ryasnyansky, R. Tugushev, and T. Usmanov, "Characterization of nonlinear optical parameters of $\mathrm{KDP}, \mathrm{LiNbO}_{3}$ and BBO crystals," Opt. Commun. 229, 403-412 (2004).

${ }^{98}$ S. C. Abrahams, J. M. Reddy, and J. L. Bernstein, J. Phys. Chem. Solids 27, 997 (1966)

${ }^{99}$ S. Juppe and O. F. Schirmer, Solid State Commun. 76, 299 (1990).

${ }^{100}$ V. I. Belinicher and B. I. Sturman, Sov. Phys. Usp. 23, 199 (1980).

${ }^{101}$ H. H. Nahm and C. H. Park, Phys. Rev. B 78, 184108 (2008).

${ }^{102}$ A. Sanson, A. Zaltron, N. Argiolas, C. Sada, M. Bazzan, W. G. Schmidt, and S. Sanna, "Polaronic deformation at the $\mathrm{Fe}^{2+/ 3+}$ impurity site in Fe:LiNbO 3 crystals," Phys. Rev. B 91, 094109 (2015).

${ }^{103}$ H. Heyszenau, "Electron transport in the bulk photovoltaic effect," Phys. Rev. B 18, 1586 (1978).

${ }^{104}$ K. M. Voit and M. Imlau, "Holographic spectroscopy: wavelengthdependent analysis of photosensitive materials by means of holographic techniques," Materials 6, 334-358 (2013).

${ }^{105}$ R. S. Weis and T. K. Gaylord, "Lithium niobate: Summery of physical properties and crystal structure," Appl. Phys. A 37, 191-203 (1985).

${ }^{106}$ H. Kogelnik, "Coupled wave theory for thick hologram gratings," Bell Syst. Tech. J. 48, 2909 (1969).

${ }^{107}$ H. Kurz, E. Krätzig, W. Keune, H. Engelmann, U. Gonser, B. Dischler, and A. Räuber, "Photorefractive centers in $\mathrm{LiNbO}_{3}$, studied by optical-, Mössbauer- and EPR-Methods," Appl. Phys. 12, 355 (1977).

${ }^{108}$ D. S. Smith, H. D. Riccius, and R. P. Edwin, "Refractive indices of lithium niobate," Opt. Commun. 17, 332 (1976).

${ }^{109}$ M. Garcia-Lechuga, J. Siegel, J. Hernandez-Rueda, and J. Solis, "Imaging the ultrafast Kerr effect, free carrier generation, relaxation and ablation dynamics of lithium niobate irradiated with femtosecond laser pulses,” J. Appl. Phys. 116, 113502 (2014).

${ }^{110}$ P. Reckenthaeler, D. Maxein, T. Woike, K. Buse, and B. Sturman, "Separation of optical Kerr and free-carrier nonlinear responses with femtosecond light pulses in $\mathrm{LiNbO}_{3}$ crystals," Phys. Rev. B 76, 195117 (2007).

${ }^{111}$ E. G. Gamaly, S. Juodkazis, V. Mizeikis, H. Misawa, A. V. Rode, W. Z. Krolikowski, and K. Kitamura, "Three-dimensional write-read-erase memory bits by femtosecond laser pulses in photorefractive $\mathrm{LiNbO}_{3}$ crystals," in 3rd International Conference on Advanced Materials and Nanotechnology, Wellington, New Zealand, Feb 11-16, 2007; [Curr. Appl. Phys. 8, 416-419 (2008)].

${ }^{112}$ K.-M. Voit and M. Imlau, "Holographic spectroscopy: Analysis of convergent phase development," Eur. J. Phys. 36, 035003 (2015).

${ }^{113}$ M. Jazbinsek and M. Zgonik, "Material tensor parameters of $\mathrm{LiNbO}_{3}$ relevant for electro- and elasto-optics," Appl. Phys. B 74, 407 (2002).

${ }^{114}$ T. Fujiwara, M. Takahasi, M. Ohama, A. J. Ikushima, Y. Furukawa, and K. Kitamura, "Comparison of electro-optic effect between stoichiometric and congruent $\mathrm{LiNbO}_{3}$," Electron. Lett. 35, 499 (1999). 
${ }^{115}$ S. Fries and S. Bauschulte, "Wavelength dependence of the electrooptic coefficients in $\mathrm{LiNbO}_{3}$ :Fe," Phys. Status Solidi A 125, 369 (1991).

${ }^{116}$ N. V. Kukhtarev, "Kinetics of hologram recording and erasure in electrooptic crystals," Sov. Tech. Phys. Lett. 2, 438 (1976).

${ }^{117} \mathrm{~S}$. Masch, "Aufzeichnung und rekonstruktion von bildhologrammen mit kurzen laserpulsen in $\mathrm{LiNbO}_{3}$," Bachelor thesis (Universität Osnabrück, 2012).

${ }^{118}$ S. Odoulov, A. Shumelyuk, H. Badorreck, S. Nolte, K. M. Voit, and M. Imlau, "Interference and holography with femtosecond laser pulses of different colors," Nat. Commun. 6, 5866 (2015).

${ }^{119}$ Y. Furukawa, K. Kitamura, A. Alexandrowski, R. K. Route, M. M. Fejer, and G. Foulon, "Green-induced Infrared absorption in $\mathrm{MgO}$ Doped $\mathrm{LiNbO}_{3}$, " Appl. Phys. Let. 78, 1970-1972 (2001).

${ }^{120}$ S. Wang, V. Pasiskevicius, and F. Laurell, "Dynamics of green lightinduced infrared absorption in $\mathrm{KTiOPO}_{4}$ and periodically poled $\mathrm{KTiOPO}_{4}$," J. Appl. Phys. 96, 2023-2028 (2004).

${ }^{121}$ S. Kato, S. Kurimura, H. H. Lim, and N. Mio, "Induced heating by nonlinear absorption in LiNbO3-type crystals under continuous-wave laser irradiation," Opt. Mater. 40, 10-13 (2015).

${ }^{122}$ Q. Wu, J. Xu, G. Zhang, L. Zhao, X. Zhang, H. Qiao, Q. Sun, W. Lu, G. Zhang, and T. Volk, "Fanning scattering in $\mathrm{LiNbO}_{3}$ at $750-850 \mathrm{~nm}$ induced by femtosecond laser pulses," in 8th IUMRS International Conference on Electronic Materials (IUMRS/ICEM), Xian, People's Republic of China, Jun 10-14, 2002; [Opt. Mater. 23, 277-280 (2003)].

${ }^{123}$ M. G. Clark, F. J. DiSalvo, A. M. Glass, and G. E. Peterson, "Electronic structure and optical index damage of iron-doped lithium niobate," J. Chem. Phys. 59, 6209-6219 (1973).

${ }^{124}$ D. A. Bryan, R. Gerson, and H. E. Tomaschke, "Increased optical damage resistance in lithium niobate," Appl. Phys. Lett. 44, 847-849 (1984).

${ }^{125}$ Y. Furukawa, M. Sato, K. Kitamura, and Y. Yajima, "Optical damage resistance and crystal quality of $\mathrm{LiNbO}_{3}$ single crystals with various $[\mathrm{Li}] /[\mathrm{Nb}]$ ratios," J. Appl. Phys. 72, 3250 (1992).

${ }^{126} \mathrm{E}$. Krätzig and R. Orlowski, "Reduction of optical damage effects in $\mathrm{LiNbO}_{3}$ and $\mathrm{LiTaO}_{3}$," Opt. Quantum Electron. 12, 495 (1980).

${ }^{127}$ T. R. Volk, M. Wöhlecke, and N. Rubinina, "Optical damage resistance in lithium niobate," in Photorefractive Materials and their Applications II, Number 114 in Springer Series in Optical Sciences, edited by P. Günther and J. P. Huignard (Springer-Verlag, 2007), pp. 165-203.

${ }^{128}$ D. Conradi, C. Merschjann, B. Schoke, M. Imlau, G. Corradi, and K. Polgar, "Influence of $\mathrm{Mg}$ doping on the behaviour of polaronic lightinduced absorption in $\mathrm{LiNbO}_{3}$," Phys. Status Solidi-RRL 2, 284 (2008).

${ }^{129}$ G. K. Kitaeva, K. A. Kuznetsov, A. N. Penin, and A. V. Shepelev, "Influence of small polarons on the optical properties of $\mathrm{Mg}: \mathrm{LiNbO}_{3}$ crystals," Phys. Rev. B 65, 054304 (2002).
${ }^{130}$ S. Enomoto and S. Ashihara, "Comparative study on light-induced absorption between $\mathrm{MgO}: \mathrm{LiNbO} 3$ and $\mathrm{MgO}: \mathrm{LiTaO}$," J. Appl. Phys. 110, 063111 (2011).

${ }^{131}$ X. Wu, S. Carbajo, K. Ravi, F. Ahr, G. Cirmi, Y. Zhou, O. D. Muecke, and F. X. Kaertner, "Terahertz generation in lithium niobate driven by Ti:sapphire laser pulses and its limitations," Opt. Lett. 39, 5403-5406 (2014).

${ }^{132}$ J. Armstrong, N. Bloembergen, J. Ducuing, and P. Pershan, "Interactions between light waves in a nonlinear dielectric," Phys. Rev. 127, 1918 (1962).

${ }^{133}$ M. Fejer, G. Magel, D. Jundt, and R. Byer, "Quasi-phase-matched 2nd harmonic-generation-tuning and tolerances," IEEE J. Quantum Electron. 28, 2631-2654 (1992).

${ }^{134} \mathrm{M}$. Houé and P. D. Townsend, "An introduction to methods of periodic poling for second-harmonic generation,” J. Phys. D: Appl. Phys. 28, 1747-1763 (1995)

${ }^{135}$ D. Feng, N. Ming, J. Hong, Y. Yang, J. Zhu, Z. Yang, and Y. Wang, "Enhancement of 2nd-harmonic generation in $\mathrm{LiNbO}_{3}$ crystals with periodic laminar ferroelectric domains," Appl. Phys. Lett. 37, 607-609 (1980).

${ }^{136}$ I. Naumova, N. Evlanova, O. Gliko, and S. Lavrichev, "Czochralskigrown lithium niobate with regular domain structure," in 4th International Symposium on Ferroic Domains and Mesoscopic Structures (ISFD-4), Vienna, Austria, Mar 25-30, 1996; [Ferroelectrics 190, $107-112$ (1997)].

${ }^{137}$ N. Evlanova, I. Naumova, T. Chaplina, S. Blokhin, and S. Lavrishchev, "Periodically poled $\mathrm{Y}: \mathrm{LiNbO}_{3}$ single crystal: Impurity distribution and domain wall location," J. Cryst. Growth 223, 156-160 (2001).

${ }^{138}$ B. Schoke, "Untersuchungen zum Ladungstransport kleiner Polaronen in reduziertem und unreduziertem $\mathrm{LiNbO}_{3}$ und PPLN:Y," Diplomarbeit (Universität Osnabrück, 2006).

${ }^{139}$ H. Mabuchi, E. S. Polzik, and H. J. Kimble, "Blue-light-induced Infraredabsorption in $\mathrm{KNbO}_{3}$," J. Opt. Soc. Am. B. 11, 2023-2029 (1994).

${ }^{140}$ V. Murk, V. Denks, A. Dudelzak, P. Proulx, and V. Vassiltsenko, "Gray tracks in $\mathrm{KTiOPO}_{4}$ : Mechanism of creation and bleaching," in 9th International Conference on Radiation Effects in Insulators (REI-9), Knoxville, Tennessee, Sep 14-19, 1997; [Nucl. Instrum. Methods Phys. Res. B 141, 472-476 (1998)].

${ }^{141}$ B. Boulanger, I. Rousseau, J. Feve, M. Maglione, B. Menaert, and G. Marnier, "Optical studies of laser-induced gray-tracking in KTP," IEEE J. Quantum Electron. 35, 281-286 (1999).

${ }^{142}$ S. Moeller, A. Andresen, C. Merschjann, B. Zimmermann, M. Prinz, and M. Imlau, "Insight to UV-induced formation of laser damage on $\mathrm{LiB}_{3} \mathrm{O}_{5}$ optical surfaces during long-term sum-frequency generation," Opt. Express 15, 7351-7356 (2007). 